\title{
HEMIPTERA CAUCASI TESSERATOMIDAE
}

S4MTHSontan DHERARY 







\section{MELETEMATA ENTOMOLOGICA}

auctore

Dre. Friderico A, Kolenati.

Fasc. II.

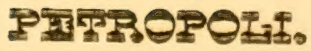

1845. 


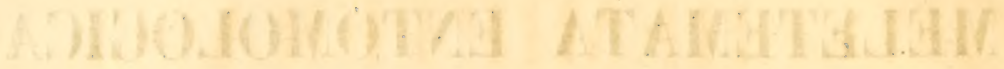

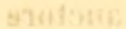

Jussu et impensis Aeademine,

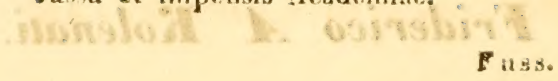

$8-2302$

ㄴ.

if 9827

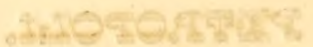

$.8+84$ 


\section{HEMIPTERA}

\section{CAUCASI.}

\section{TES S E R A T O M I A E}

MONOGRAPHICE DISPOSITAE

i) 4

F. Holenai.

Acchont TABUias IX.
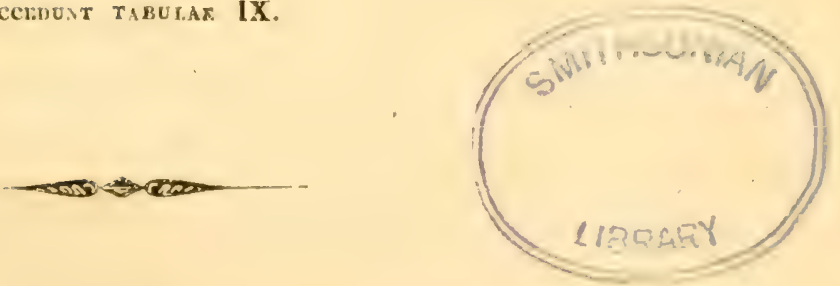

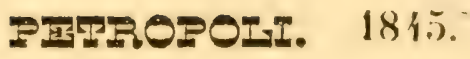

Typis Imperialis Academiac Scientiarum. 
Cimices sive expellant mundi, sive spernant ignari, sive tracture nolint gnari: mihi scientias augendi voluntas! 


\section{CELSISSIMO}

di:

\section{E X C E L L E IN T ISS I MO}

\section{PPI IVIIPI}

\section{Atlichaeli}

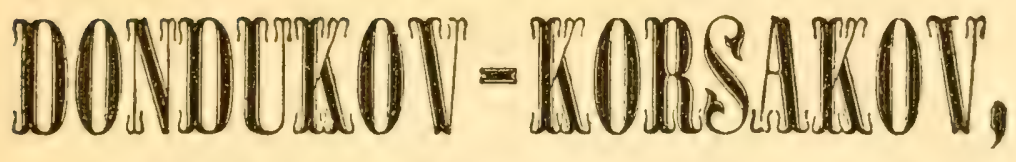

IMPERATORI AUGUSTISSIMO A CONSILIIS INTIMIS IMPERIALIS ACADEMIAE SCIENTIARUM PETROPOLITANAE PRAESIDIS NUNERE FUNGENTI COLLEGII DE DIRIGENDIS SCHOLIS PRIMARII SOCIO

ORDINIY ROSSICORUM S-tae ANNAE I-mae CLASSIS CUN CORONA IMPERIALI, S-ti STANISLAI I-mae, S-ti VLADHIIRI IV-tae CLASSIS CLY LENYISCO

ET REGII BORLSSICI PRO MERITIS MILLTARIBUS EQUITI

ETC. ETC. ETC. 


\section{VIRO GENEROSISSINO}

GR.ITITUDINIS ANIMIQUE

PII MONITIONI SACRUM

ESSE PETIT

DRVOTRSSMUE

Autor. 




\section{P a e i t i c}

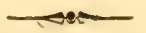

Perfectissima scientiae naturali ab illo tantum praeberi opera, qui omni studio in uno tantum versatur forulo, nullo negare possum pacto; ast mirum in modum exar descere in terris peregrinis omnis generis literarum amorem, ut eliam foeda tractandi evadamus proni, quis naturae scrutatorum infitias ibit! -

Iline in Caucaso et Transcaucasia horis nonmullis a negotiis botanicis aliisque liberis IIemipteris etiam imperlitis trecentas collegi species, quarum ducentae quinquaginta ad Heleropterce et quinquaginta ad Homoptere spectant. Harum evaserunt species novae quindecim, illarum quinquaginta quinque. Nihilominus tamen Caucasum et Transcaucasiam, plantis et animalculis praedivitem, numerum adhuc continere IIemiplerorum duplum e sequentibus deducere audeo:

1. e multiplici plantarum cohorte,

2. quod aves et reptilia egestate tantum coacha cimices devorent,

October. 184:5. 


\section{$-2-$}

3. quod cinicum tarris ef imaginitus anlar conreniat solis et

4. ex cirenastantiis plura mibi colligendi sompo soppias obstantibas. -

Nostro aeto duo millia et centum cinuicum speries innotescunt, quarum scrcentse in Europa inveniumbur, et quidem:

In Lapponia teste $D^{\circ}$. Zstterciedt 128 species.

\begin{tabular}{|c|c|c|c|c|}
\hline Suecia & D & D & Fallén & $2+1$ \\
\hline Caucaso & D & $\pi$ & Kolenati & 250 \\
\hline Bohemia & $D$ & s & Fieber & 300 \\
\hline Anglia & D & $x$ & Stephens & 337 \\
\hline Ielretia & $D$ & $D$ & Merer & 360 \\
\hline
\end{tabular}

Si e plantaram distributione ad cimicun onpiam concludere licet, Petropoli tix uumerum 200 specierua! inrenies. In imperio Rossion tam entenso, et salu ef climate diterso, integrum numerum Europseorum cimicum accipere fas est, nisi alhuc Asiatici et haic imperio proprii rem amplient. Penitus in Rassia neglecta hurum insectorum classe conclusivuibus nithil adtuc existit oertius; quam ob rem primo hujusaodi is iuperio Ihessico cpuscula de Hemipleris benervil venime detis, eciam atque etiam petit

Auctur.

Fetropoli mense Augusto 1875. 


\section{L i t e r a I u r a.}

1. Bauhin. J. Historia admirabilis fontis Bollensis in ducato Württenbergico, cum plurinis figuris insectorum. Nontisbeligardi $1598-1660.4^{\circ}$.

2. Strolelherger. J. S. Tract. in quo de cocco Baphico et quae inde paratur. Jenae. 1620. 4?.

3. Kirsten. G. Vint au monde le ro janvier 1613 à Stettin et y mourut le 4 mars 1660. Exercit. Phytophilolog. de Colocynthide et Cocco. Stettin. 1651. 4".

4. Bondel. G. F. Diss. 11 de Navigatione Salomonis in Ophir, de purpura et cocce. Hamburg. 1660. $8^{\circ}$.

5. Hire. Description d'un Insecte qui sattache à quelques plantes etrangeres et principalement aux orangers, Coccus. Mémoires de l'Academie des Sciences de Paris. T. II. p. 116. t. X. p. 10-i'. 1666 $\mathbf{- 1 6 6 9 .}$

6. Lister. M. Letters concerting the kird of insects Kermes. Philosoph. Transact. n. 71. p. 2165-2166. Badd. I. p. 311. à $515 .-$ Suite Philus. Transact. n. T3. p. 2196-2197. - Lesken. Uibers. I. Bd. I. Th. p. 90. - Suite Philos. Trans. 1672. n. 87. p. 5059 - 5080 . - Lesken Lebersetz. I. Bd. II. Th. p. 48. Anno 1671 . 
7. Meyer. A. De Ricadis Indicis. Ephem. Miscel. Acad. Tat. Curios. dec. 2. obs. 49. p. 124. Anno 1687.

8. Ment:cl. J. C. De Cicadis et aliis insectis canoris, primo de cicada Bononiensi. Miscell. Acad. Nat. Curios. Dec. 2. arn. 6. obs. 48. p. 119. Anno 1687.

9. Sedilcau. Description d'un insecte qui sattache it quelques plantes étrangères, et principalement aux orangers (Coccus Hesperidum). Mém. de l'Ácad. des Sc. de Puris. 1692. p. 8-11. - Ihid. 1666, 1667-1699. t. 10. p. $10-14 .-$

10. Valentini. M. B. De vermibus salivatoriis, unde spuma cuculi (Aphrophora). Miscell. Acad. Nat. Curios. 169'. Dec. 3. A. 2. p. 183.

11. Plumier. C. Réponse ì Pomet sur la Cochenille. Journal des Savants. t. 22. p. 212. $169 \%$.

12. Reyger. G. Von der Erzeugung der Buatläuse. Danzig. Gesell. Abhandl. 169'. Th. 2. p. 29'-301.

13. Richter. O. F. Dissert. de Cochinilla resp. Friedel. (1 pl. représentant opuntiam) Upsaliae. 1701. "4". et $8^{\circ}$. Deutsch. Uebers. Upsala 1703. $8^{\circ}$.

14. Peticer, James. Gazephilacium naturae et artis. Six decades, arec $100 \mathrm{pl}$. In fol. London. 1702-1711.

15. Hire (de la). Observations sur les Pucerons. Mém. Acad. des Sc. de Paris. 1703. Hist. p. 16. edit. in 8. p. 19.

16. Sedilcau. Nouvelles remarques sur les Insects des orangers. Mém. de l'Acad. des Sc. de Puris. 170't. p. 45 -48. édit. in $8^{\circ}$. p. 60 .

17. Plumier. C. Réponse à Richter sur la Cochenille. llemoire pour lhist. de sciences et beaux-artes. 170\%. Sept. p. 221. 
18. Langius. J. Diss. de Cochenilla in "iperum. In fol. 1704. t. 2. p. 427.

19. (Anonymus). Sur la cochenille. Mémoires de l'Academie des Sciences de Paris. 1704. p. 10. Deutsch. Anmerkungen über alle Theile der Naturlehre. I. Th. p. 51. - II. Th. p. 32.

20. Poupart. F. Des écumes printanniers. Mém. de l'Acad. des sc. de Paris. 1705. p. 127-127. Edit. in $8^{\circ}$. Mém. p. 162. - Journal des Savants. t. 21. p. 550.

21. (Anonym). Sur la Cochenille. Mémoire de Trévoux. Observ. sur toutes les parties de la Physique. t. 11. p. 35. 1707.

22. Lister. M. Systema entomologiae; à la suite de l'ouvrage sur les insectes de Ray. London. 1710. $4^{\circ}$.

23. Mursilli. L. F. Annotazioni intorno alla grana de tintori della kermes in una lettera al Vallisnieri (avec. 3. planch.), saggio fisico dell' istora del mare. p. 76. Venezia. 1711. 4. - En latin. dans les Ephem. nat. curios. vol. 3. app. p. $33-48$. avec figure. Eu Allemand dans le Crells neu chem. Archiv. 1. B. p. 348.

2'. Garidel. P. J. Histoire des Plantes qui naissent aux environs d'Aix et dans plusieurs autres endroits de la Provence. In fol. Aix. 1715. In fol. Paris. 1723. Dans cet ouvrage l'auteur decrit te Chermes qui sert à la teinture.

25. Bradley. R. A. Philosophical account of the Works of nature. London. 1721. 4".

26. Fisch. J. L. Beschreibung von allerlei Insecten in 
Teutschland. VIter Theil in $4^{\circ}$. Berlin. 1727 mit Abbildungen. VII. Th. 1723.

27. Ruyscher. 11. Naturlike historis van de Couchenille beweezen mest anthentique documentum. Texte hollandais et français, aree 1 . planche. In $8^{\circ}$. Amsterd. 1729. - Deutsch. Physikal. Belustigg. I. Bd. p. $43-57$. p. $96-107$. p. $188-195$. p. $367-377$. p. $546-559$. p. $654-671$.

28. (Anonrm). The natural history of Cochineal, being an account of a Book entitled. Amsterdam. 1729. - Trans. Philos. n. 413. p. 264. - Badd. 9. p. 40.

29. Southall. J. Treatise of Buggs, arec $1 \mathrm{pl}$. in 8 ". London. 1730. - Deutsch uibers. Hamburg. 1737. - Berlin 1742.

30. Breynius. J. P. Historia naturalis Cocci radicum tinctorii (c. tabul. cols). In $4^{\circ}$. Dantsig. 1731 ou Gedani. Comm. Toriberg. p. 413. - Act. Erudit. p. 406. - Ephem. Nat. Curios. vol. 3. Appendix. p. $1-27$.

31. Frisch. J. L. Beschreibung von allerley Insekten in Teutschland. X. Theil. Berlin. 1732.

32. Breynius. J. P. Corrigenda et emendanda circa generationem Cocci radicum in $4^{\circ}$. Comm. litt. Norimberg. 1733. p. 11-14. - Act. Erudit. Lipsiae p. 167-171. - Ac. Acad. Nat. Curios. vol. 3. p. $28-32$. - En anglais. Philos. Transact. vol, 37. n. 426. p. $444-447$.

33. Trew, C. J. De cimicibus mali persicae et cerasi. Commerc, lit, Norimberg. 1734. p. 361.

34. Tres, C, J, De Insectorum quodam genere (cher- 
mes et coccus). Commerce litterar. Norimberg. 1734. p. $361-363$.

35. Reuumur. Mémoires pour servir à l'historie des Insectes. In $4^{\circ}$. Paris. 173'-1742. VI Vol. In vol. III. cimic.

36. Trew. C. J. Peculiare quoddam quercus excrescentiarum genus (chermes). Commerc. litt. Norimberg. 1735. p. 339-341.

37. Linné. C. Systema naturae $I^{m a}$ edit. in fol. Lugduni Batavorum. 1735.

38. Bradley. R. A. Philosophical account of the Works of nature. London. 1739. $8^{\circ}$.

39. Bose. G. M. Diss. Otia Witsebergensia. Resp. Leugerken. In $4^{\circ}$. Wittemberg. 1739.

40. Bergen. C. A. Progr. le Alchimilla, gramineo et baccis quae circa radices ejus reperiuntur. In $4^{\circ}$. Francf.-O- 1739. .

41. De Geer. C. Beskriforing pả en Insect, som lefver uppä mäst alla Orter ock trän uti et hwitt skum, ock kallas. Cercope. Vetensk. Acad. IIandl. p. 221, 222-236. 1741. - Deutscl. p. 257. - Latine. Analecta Transalpina. t. 1. p. 166-176. - Daus ses mémoirés t. 3. p. 163-180.

42. Rossi. P. Fauna Etrusca insectorum. Liburni. 1741. $4^{\circ}$. cum fig.

43. Burchard. E. F. Epistola ad C. Linnaeum de Cocco Polonico. Act. Soc. Upsal. 1742. p. $53-78$. Deutsch. Neu. Hamburg. Nagaz. 23. St. p. 481 , 482-496. - 24. St. p. $499-528$.

44. Bonnet. C. Traité d'Insectologie, ou Observations sur les Pucerons, (avec. 4. pl.) 2. vol. in $8^{\circ}$. Paris. 1745. 
45. Linné. C. Fauna Suecica. IIolmiae ed. I. 1746. Ulolmiae ed. II. 1761.

46. Linné, Charles von. Licte Matken Fräu China (Fulgor ( $)$ Vetensk. Acad. Ifandl. 1746. p. 60-66. Deutsch. p. 61 67. - Latine. Analecta Transalpina. t. 1. p. $475-479$.

47. Geer. Carl de. Lyckte - masken frâu China (Candelaria). Vetensk. Acad. IIandl. 1746. p. 60-66. - Analect. Transalp. t. 1. p. 475- 479.

4.8. Rösel von Rosenhof. Monatliche Insektenbelustigungen. 4. vol. in $4^{\circ}$. Niirnberg. $174.6-1761$. In volumine II. cicadae, in III cimices.

49. (Avonym.) Bekrifning på en Insect of ett nytt slïgte kallad Physapus. avec. fig. Vetensk. Acad. Handl. 174.7. p. 1. - Deutsch. p. 3. - Latin. in Analectis Transalpinis. t. 1. p. $277-281$. Dans ses mémoires. t. 3. p. 4-11.

50. Bergen. C. A. Epistola de Alchimilla ejusque coccis $\left(2 \mathrm{pl}\right.$.). In $4^{\circ}$. Francofurti-O-1748.

51. a. Linné. Systema Naturae. 17ł8. Holmiae.

51. b (Anonym?) Nagre rön och försok augaende honungs dagge, samt tanskar och Anmarkingar des röfser. Vetenskaps. Academ. Ilandl. 174.8. p. 236. - Deutsch. iü. p. 2 \%.

52. Delius. II. F. Diss. de Purpura et Coccinella. Resp. Schauer. In 4". Erlang. 1753.

53. Linné. C. Chinensia Lagerströmiana. Diss. Resp. Ollhelius. In $\mathrm{t}^{n}$. IIolmiae. Amaenit. Acad, t. \%. $175 \%$.

54. Kalm, P. Beskrifning pâ et flags Gras-IIoppor, uti Norra America, cicada septemdecim? Vetenskaps. 
Akad. Handl. 1756. p. 101-116. - Deutsch. p. 94. - Fuessl'ys N. Entom. Magaz. 3. Bd. p. 4.1.

55. Martini. Chr. Osservazione intorno ad una specie di Cimici selvatiche non alate. Hemorie sopra la fisica in Luca. 1759. t. I. p. $247-267$. - En francais dans le Nouvelliste économe t. 3! p. 117. - En allemana dans le Hamburg Magazine 26. Bd. p. $432-447$.

56. Limné. C. Svensk. Coccionell. Vetensk. Acad. IIandl. 1759. p. 26. - Deutsch. p. 28.

57. Sulzer. J. II. Die Kennzeichen der Insekten. \&". Zürich. 1761. mit col. Kupfern.

58. Geoffiroy. Llistoire abregée des Insectes, qui se trouvent aux environs de Paris. $4^{\circ}$. Paris. 1762.

59. Scopoli. J. A. Eutomologia Carniolica. Vindobonac. 1763. $8^{\circ}$.

60. Saurages. P. A. (de la Croix de). Observation sur l'origine du miel. Nimes. 1763. Journ. de Physique t. 1. p. 187-197. - Berlin. Sammlung. 6. b. p. $453-4.79$.

61. Collinson. P. Some observations on the Cicada of North-America. Philos. Trans. 176\%. p. 65.

62. Modeer. A. Nägre mark vardigheter hos Insectet. Vetenskap. Academ. Ilandilingar. 176\%. p. 41-47. - Deutsch. p. 43.

63. Nodecr. S. Oekonomische Beschreibung der Kirchspiele halltorp und wertorp. Vetensk. Ac. IIandl. 1765. 29. Band.

64. Erxleben. J. C. P. Begattung der Cochenillen. Ulannöv. Magaz. 1765. st. 90. 
65. Schacfer. J. Ch. Icones Insectorum circa Ratisbonam indigenorun. Vol. I. Rutisbonae. 1766. '4".

66. Ellis. J. An Account of the Male and liemale Cochineal Insects (avec figures) Philos. Trans. 1766. vol. 52. p. 661-667. - En français. Journal des Savants. 1767. juin. p. 251.

67. Linné. C. Systema naturae. Edit. XII. 8". Holmiae. 1767.

68. Bandaroy. A. D. F. Observations sur une Cigale prise à Denainvilliers. Mém. de l'Acad. des Sc. de Paris. 1767. Hist. p. 22.

69. Canals. Y. Marti (J. P. Don). Mémorias sobre la grana Kermés de Espagna, que es el Coccum, o Cochinilla de los antiquos (avec. 1. pl.). In $4^{\circ}$. Madrid. 1768:

70. Schaeffer. J. Ch. Icones Insectorum circa Ratishonam indigenorum. Vol. II et III. Ratisbonae. 1769. 4".

71. Gleichen. W. F. (von, surnommé Russworm). Versuch einer Geschichte der Blatıläuse. Blattläusefresser des Ulmenbaumes. In 4\%. Nürnberg. 1770.

72. Richardson. W. Observations on the aphides of Linnaeus. Phil. Trans. 1771. t. 61. p. 182-194.

73. Pallas. P. S. Spicilegia zoologica. Fase. IX. Berolini. 1772. Cicadella et cimex unicus.

74. De Géer. Mémoires pour servir á l'histoire des Insectes. avec fig. Stockholm. 1773. Vol. III. Cimices.

75. Goeze. J. A. E. Beschreibung des Cicada sanguinolenta mit Anmerkungen über das Cicarlen-Geschichte mit 1 taf. Naturforscher. 1775. 6. St. p. $4-68$. 
76. Fuessly. J. C. Verzeichniss der bekannten Schweitzerischen Insekten. in $4^{\circ}$. Zürich. 1775.

77. Sulzer. J. II. Abgekürzte Geschichte der Insekten. Winterthur. 1776. $4^{\circ}$. II Theile mit Kupfern.

78. Sparrmann. A. Bekreifning på Cimex paradoxus, en ny Insects ifrïn caput Bonae spei. Vetenskiaps Acad. Ilantl. 1777. A. p. 234-238. - Trad. allem. 29. Bd. p. 217.

79. Diury. Illustrations of natural history. London. $1770-1782$. 3 vol. in $4^{\circ}$.

80. Kerner. J. S. Naturgeschichte des Coccus Bromeliil, oder des Ananasschildes ete. mit 1 Taf. in $8^{\circ}$. Stuttgard. 1778.

81. Modeer. A. Om fäst flyet. Götheborska Vetensk. Handling. St. 1. p. 1. 1778.

82. De Geer. Uibersetzt von J. A. Ephraim Götze. III. Band. Nürnberg. 1780.

83. Stoll. C. Natuurlyke en naar 't leeven Naauwkeurig gekleurde Afbeeldingen en Beschryvingen der Cicaden en Wantzen. Te Amsterd. Vol. I-VI. (inclusive). 1780.

8'v. Schrank. Fr. Enumeratio Insectorum Austriae. Augustae Vindelicorum. 1781. $8^{\circ}$.

85. Schrank. Von der Cicada sanguinolenta. Schriften der Berliner Ges. Naturf. Freunde. 1781. Bd. 2. p. 307.

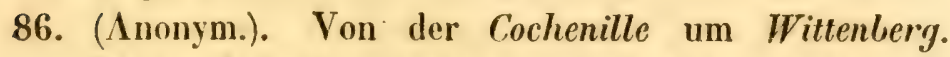
Witt. Wochenbl. 1781. 1. b. p. $193-381$.

87 a. Nevillau. B. Remarques sur la Punaise des jardins qui poursuit les chenilles. Rasier. Journal de Physique. 1782. 
87 b. Bose. Journal de Physique. 178'. Dorthesia. (Cicadae).

88. Thunberg. Dissertatio entomologica novas insectorum species sistens. 4?. Upsaliae. 1783.

89. Bierlkander. C. Anmarking om socker pả Gran. Vetensk. Acad. nya Handl. 178\%. p. 238 - 2\%.0. - Crell'. Chemische Annalen 1786. I. Band. p. $351-353$.

90. Stoll. Répresentation exactement coloirée d'aprés nature des Cigales et Punaises. Ansterd. 1784. Vol. VII - X (inclusive).

91. (Anonym.) Beobachtung über die Blatliüuse Riems und Löwes. - Phys. ökon. Zeit. 1785. p. 251. Journal für die Gärtnerey. 1785.

92. Swederus. N. S. Et nytt Genus och fentio nya species af Insect. (Macrocephalus cimicoides) Vetenskaps. Acad. nya Ilandl. 1787. p. 181-290.

93. Linck. J. W. Diss. de Coccionellae natura, viribus et usu. cum tab. in $4^{\circ}$. Lipsiae. 1787.

9\%. Blenonsille (N. J. Thiéry de). Traité de la culture du nopal et de l'éducation de la cochenille dans les "colonies francaises de l'Amérique, avec plusicurs planches colorićes. 2 vol. in $8^{\circ}$. Cap Trancais 1787.

95. Stoll. Vol. XI. 1787.

96. Linné. Systema naturae edit. 13 par Gmelin in 8 ". Lipsiae. 1788.

97. Oedmann. S. Berättelse om vöggloss fundue i skogar. Vetenskaps. Acad. nya Handl. 1789. p. 76, 77, 78. - Deutsch 1789. p. 69. -

98. Carlson. G. Tillaegning vid föregäende rön. V(1- 


\section{$-13-$}

tenskaps Acad. nya Ilandl. 1789. p. $78-79$. Deutsch. p. 79.

99. Panzer. G. F. Fauna insectorum Germaniae. Niurnberg. 1792. 12 Fasciculi.

100. Hlodlecr. A. Anmärlingar angảende det sâ kallade manna foliata, eller manna di fronde. Vetensk. Academ. Ilandl. 1792. p. 161-166.

101. Donovan. E. The natural history of british insects. cum tab. col. London. 1792. Vol. I. - 1793. Vol. II. - 179\%. Vol. III. a pl. 1 ad 108 (inclusive).

102. Fubricius. J. Ch. Entomologia systematica in IV. Tom. Iafniae. $8^{o} .179 \%$.

103. Latreille. P. A. Mémoire sur la Phalène culiciforme de l'éclaire. Nagas. encyclopédique. 1795. No. 8. t. 4. p. $30 \%$.

10\%. Donovan. The natural history of british insects. 1795. Vol. IV. a pl. 109-14/\%. (inclusive).

105. Latreille. P. A. Deseription du kermès mâte de l'orne. Magas. encycl. 1796. t. 2. p. 146. Rémprimée à la suite de l'llistoire des Fourmis. 1802. p. $326-332$.

106. Donovan. 1796. Vol. V. a pl. 14.5-180 (inclusive). 107. " 1797. " VI.» ", 181-216 " 108. " 1798. "VII." " $217-252$ "

109. Falricius. J. Ch. Supplementum Entomologiae systematicae. in $8^{\circ}$. Ilafnice. 1798.

110. Marshum. T. Further observations on the coheat insects. Trans. Soc. Linn. of London. 1798. t. 4. p. $22 \%$.

111. Latreille. P. A. Mémoire sur une nouvelle espéce de Psylle (Livia). Bull. de la Soc. Philom. 1798. 
t. 1. an 6. p. 113. - Rëimprimé ì la suite de l'llist. des fourmis p. 321.

112. Donovan. 1799. Vol. VIII. a pl. $253-288$ (inclus.).

113. Coquebert. J. $\Lambda$. Illustratio iconographica insectorum, quae in museis Parisinis observavit et in lucem edidit. J. C. Fabricius, in fol. Paris. 1799.

114. Donovan. 1800. Vol. IX. a pl. 289-324 (inclusive).

115. Schellenbery. Das Geschlecht der Land- und Wassersanzen nach ihren Familien, mit $14 \mathrm{col}$. Taf. $8^{\circ}$. Zürich. 1800.

116. Koelreuter. J. G. Nachricht von einer Schwarzbraunen vanze, die sich die Roth-Tannenzapfen zu ihrem Winterlager Erwählt und gegen diese Jahreszeit den kreuzvögeln zur taeglichen speise dient. Comment. Acad. Theodoro-Palatinae. 1800. vol. 3. p. $62-68$.

117. Donovan. 1801. Vol. X. a. pl. $325-160$. (inclus.).

118. Lamark. J. B. Systéme des animaux sans vertìbres. Paris. 1801 . I. vol. in $8^{\circ}$.

119. Hausmann. J. F. L. Bemerkungen über Lygeus apterus. Fabricii. - Magaz. zur Insectenk. 1802. I. Bd. p. 229.

120. Hausmann. J. F. L. Beyträge zu den Materialien für eine künftige Bearbeitung der Gattung der Blattlünse. Magaz. zur Insektenkunde. 1802. 2. Bd. p. 426.

121. Curlis. W. Observations on the Aphide (c. pl. col.). Trans. Soc. Lin. of. London. 1802. t. 6. p. $75-94$.

122. Schrenk. F. Fauna boica. 8". Ingolstadt. 1801, 1802, 1803. - Tom. III. cum insectis. 
123. Illiger. J. Ch. G. Magazin für Insektenkunde. 7. vol. in $8^{\circ}$. Braunschweig. 1801-1807.

124. Fubricius. J. C. Systema Rhyngotorum. I. vol. in $8^{\circ}$. Brunsvigae. 1803.

125. Wolff. J. F. Icones Cimicum descriptionibus illustratae in $4^{\circ}$. Erlangae 1804. cum 20 tab. col.

126. Palisot de Beauvois. Insectes recueillis en Afrique et en Amérique in fol. avec pl. enlum. Paris. 1805.

127. Schreibers. C. Descriptions of some singulare Coleopterous insects avec $2 \mathrm{pl}$. col. Transact. Linnean. Society. 1806. t. 6. n. XVI. p. 185-206.

128. Donocan. 1806. Vol. XI. a. pl. $361--396$ (inclus.j.

129. Latreille. Genera crustaceorum et inseetorum. 4 vol. in $8^{\prime \prime}$. cum fig. Paris. $1806--1807$.

130. Dumérille. Zoolog. analytique. 1806. IIemiptera et IIomoptera.

131. Donosan. 1807. Vol. XII. a pl. $397-432$ (inclus.). 132. Fullén. C. F. Monographia cimicum Svecia. 1 vol. $8^{\circ}$. Ilafiniae. 1807.

133. Donovan. 1808. Vol. XIII. a pl. $433-468$ (inclus.). 134. Virey. J. J. Recherches sur l'insecte de la gomme laque Coccus. Journ. de pharmacie des Sc. accessoires. avril. 1810. - Dict. des Sc. Médic. art. Cochenille. t. 10. p. 193.

135. Virey. Pucerons dès Therèbinthes. Dictionaire des Sc. Nédic. t. 9. p. 307. 1810.

136. Donoran. 1810. Vol. XIV. a pl. $469-504$ (inclusive).

137. Kirby. WV. Achillus flammens. Transact. Linnean Society of London. 1810. t. 12. p. 23. 
138. Olisier. A. G. Observations sur les genre Fuigore. Journ. d'llistorie nalurelle. 1810. t. 2. p. $31-3$ 't.

139. Panser. G. F. Fauna Insectorum Germaniae. a. 12 ad 109 fasc. Nürnberg. 1810.

140. Truchet. I. Traite complet du Chermes (avec 2 pl.) $8^{\circ}$. Paris. 1811.

14. Donosan. 1811. Vol. XV. a pl. 505-5't0 (incl.).

14.2. Bureil. J. On the Lygeus micropterus. Trans. of the Entom. Soc. of London. 1812. p. 73.

1'3. Hassorth. A. II. Observations ou the Coceus vitis. Trans. of the Entom. Soc. of London. 1812. p. 257.

14. Germar et Ahrens Fauna insectorum Europae. Halae. 1813.

145. Donocun. 1813. Vol. XVI. a pl. 5+1-576. (inel.).

1'+6. Fischer. Gotthelf. Zoognosia tabulis smopticis illustrata. Vol. I. edit. 3tia. Mosquae. 1813. $4^{\circ}$. Hcmipterorum genera. p. $299-307$.

147. Fallén. Iydrocorides et Nuucorides. Sreciae. $8^{\circ}$. Lund. 1814.

148. Fullén. Specimen novan Hemiptera disponendi methodum exhibens. Lund. 1814.

149. Leach. Zoological Miscellany. (Myctis). Coreides. 181 4.

150. Ky ber. J. F. Einige Erfahrungen und Bemerkungen üher Blattlüuse. IIag. Ent. Germar. II. IIft. p. 1 - 39 .

151. Germar. E. F. Magazin für Entomologie. Halle. $8^{\circ} .1815$.

152. Leclere. Ohserrations sur la corne du Psile de Duse, praesentees á litcatemie des Sciences. 1815.

153. Lumarli. J. B. Histoire naturelle des animaux sims vértèbres. 7. rol. in $8^{\circ}$. Puris. 1815-1822. 
15\%. Leach. W. E. On the genera and species of proboscideous Insects. Coccides. 1817.

15.5. Leuch. On the classification of the natural tribe of Insects Totonectides, with deseriptions of the British epecies. Trans. Soc. Linn. of London. t. 12. 1'. part. p. 10. 1817.

156. Inffimainsegg. In Wielemanns Zoolog. Magaz. (IIemityphlus). Lygaeites. 1817.

157. Leach. Encrclopedie. Brewsters. Edinhurgh. 1817 et 1818 .

158. Leteh. I.inn. Trans. XII. Mipidue et Gotmectue. 1818.

159. Kirby. In the Transactions of the finnean Society of London. Nit. 1818. Fulgorellde.

160. Fullén. C. H. Monographia rimicum Srecian. IIafnicle. $8^{\circ} .1818$.

161. Germar. E. H. ef Zinehen. Magarin der Entomo-

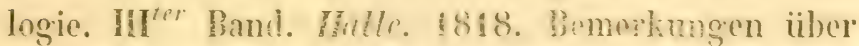
einige Gattungen do: Cidudaten. wan p. $17 \overline{7}-2.27$.

162. Fallés. Ilmiptert Srocias. Lund. 10 Hefte. 1819.

163. Suhlberg. C. R. Onservationes quaedam historiae Notonectidum. etc. 4". Aboue. $\$ 819$.

16. Kirby. W. The characters of Otiocerus, and Inotia the new Genera of hemipterous lnsects, belonging to the family of cicuda with a description of several species (avec i pl. col.). Transact. Linnean Society of London. t. 13. $1^{r r}$ part. 1821. p. 12. - Estrait, bulletin de la Socicti philomatique, août 182.2. p. 119. - Annales des Sciences naturelle. t. I. p. 192. avec planche.

165. Germar und Zincken. Magazin der Entomologie. IV $^{\text {ter Band. }} 8^{\circ}$. Ilalle. 1821. Cicudarien von p. $1-106$.

October. 1845. 
166. Ilugenbach. J. J. Symbolae Fannae Insectorum Helvetiae. Fase. I. kl. $8^{\circ}$. cum tab. col. 15. 1822. Basileae.

167. Thumberg. C. P. Disscrtalio Entomologica de Hemipleris rostratis capensibus. in $4{ }^{\circ}$. Upsalae. IV partes. 1822.

168. Eschscholtz. Entomographien in den naturwissenschaftlichen Ahantlungen aus Dorpat. 8". Berlin. 1822 et $\$ 823$.

169. Hurdwick. Th. Ot three new insecte from Repaul. (Panorpe, Gerris, Pangonie) avec 2 pl. noires. Trans. Soc. Linn. t. 14. part. 1" ${ }^{r e}$ p. 131. 1823.

170. Hardscick. Th. Deseription of a new genus of hemipterous Insect. (Thom. Rerfuviartae, Plilocerus). Trans. Soc. Linn. of London. 1823.

171. Kirby. In the Transactions of the Linnean Society of London. XIII. 1323. Otiocerus (Fulgorellae).

172. Dufour. L. Anatomic de la Raralre linéenne et de la Népe cendrèe, avec 3 pl. lithographiées. - Annales générales tes Sc. physiques. 1. 7. p. 194. pl. 105107.1823.

173. Worth. J. Sur un Insecte irés destructeur du pêcher. Journ. ot the Acat. Nat. Sc. of Philadelphia. vol. 3. ก. 7. 1823.

174. Curtis. John. British Entomolong. London. $8^{\circ}$. cum tab. col. 1824. Vol. I. a pl. $1-50$ (inclus.)

175. Blot. Mém. Soc. Limn. Caen. (Myzoxyle). Aphidui. $182 \%$.

176. Thiébaut de Berneaud. Description d'une nouvelle espèce de Dorthesiu existante aux environs de $P(t-$ ris. Mém. de la. Soc. Linn. de Paris. 1824. t. 3. sept. p. 285. avec fig. 
177. Say. Th. Hémiptères el Orthoptères recueillis dans la darniere expedition anx montagnes Rocheuses. Journal of Acarl. See. nat. of Philadelph. t. 4. 2' partie. 182\%. p. $307-345$.

178. Dufour. L. Description d'une nouvelle espéce de Coccus. Extrait des Annales dies Sc. natur. 182\%. juin. t. 2. p. 203.

179. Descourtil. Th. Mímoira sur un nouveau genre d' Insecte de lordre des lémiplères (avec fig.). Ném. de la Société Linn. de Paris. t. 3. p. 293. $182 \%$.

180. Curtis. J. Brit. Ent. 1625. Vol. IT. a pl. 51-98 (inclusive).

181. Leuch. (Risso). Ilistoire naturelle des principales profuctions de l'Lurope meritionale. Phoralis ( Aphidii). 1825.

182. Lepelletier (de St. Largean) ot Serrille. Encyclop. meth. 1825.'X. Hemipler'a et Ileteroptera.

183. Thunberg. C. P. Dissertatio insectorum hemipterorum tria genera illustranie. 8". Upsaliue. 182.5.

184. Dalman. J. W. Om naegra Svenska arter of Coccus. K. Vetensk. Aca!. Handl. 2 part. p. 350 in 4. Stockholm. 1826.

185. Duvur. A. Nouvelles Recherches sur l'histoire naturelle des Pucerons. Némoires du Mliseum d'llistoire naturelle de Paris. t. 13. p. 126-140. Annales de Sc. Nat. t. 5. p. 22\%. - Bull. de la Soc. Philom. avril. 1825. 1826.

186. Iahn. Icones ad monographiam Cimicum. Norimberg. 1825.

187. IIoffmannsegy. In Encyclopedie méthodique. 1825. X. Cicadellae. 
188. Killel. Mémoire sur les Pucerons. t. 5. - Annales de la Sociéte Linn. de Paris. mai. t. 5. p. 133. 1826 .

189. Curlis. Brit. Ent. 1826. Vol. III. a pl. $99-146$ (inclusive).

190. Fullén. C. l?. Supulementum Cinicidum Sueciae. Resp. C. J. Hofverberg in $8^{\circ}$. Lund. 1826.

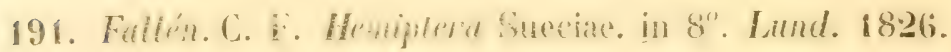

192. Firha. Intoutuction in Entonolog. ele. (Bhinuchus) Ligacites. 1826.

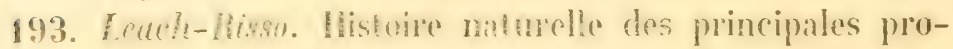

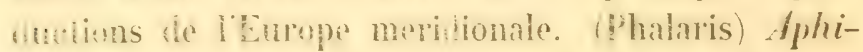
dii. $18 \div 6.1827$.

19\%. Curlis. Brit. Ent. 1827. Vol. IV. a. pl. 147-19\% (inclusive).

195. Brandt. J. F. et Rutzehurg. T. T. C. Medizinische Zoologie. II Bde. in $4^{\circ}$. mit 69 illum. Kupfertafeln. Berlin. 1827-1833.

196. Zcliestedl. I. H. Watua insectorum Lapponiae. Pars. 1. 8\%. Hummone. 1928.

197. Curtis. Brit. Ent. 1828. Vol. V. a pl. 159-241 (inclusive).

198. Henchen. C. Despriptions of a new genus of IIemiptere and a Species of IIngeter, avec figures. Znol. Journ. 1828. t. V. p. 35.

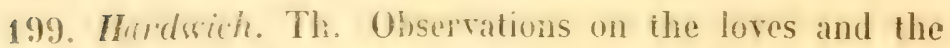
Ints, ant the Aplides. arec. fig. Zool. Journal. 1828. t. IV. p. 113.

200. Sehilling. Hemijtere Heteroptera Silesiae, adjectis icmutum tobulis. p. 31-32. In den Beiliägen zu

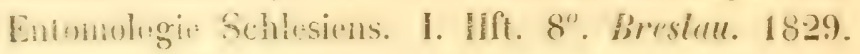


201. Fallén. C. F. IIemiplera Sveciale. 8n. Londini Gothor'tur. 1829.

21)2. Curtis. Brit. Ent. 1829. Vol. VI. a pl. 22.2-289) (inclusive).

203. Stephens. d. [1. Systematic Catalogue of Brilish Insecis. 8". London. 1829. Hemipler'e a p. 335-369.

204. Guilding. L. An account of Margarates, a new genus of Insects found in the neighbourhood of Ants Nest. Traus. Soc. Linn. of London. 1829. $t$. 16. $1^{r p}$ part. p. 115.

2115. Curlis. Brit. Hut. 1830. Vol. VII. a pl. 290--337 (inclusive).

206. Blot. F. Memoire sur le Puceron lanifère. in $8^{\circ}$. Caen. 1830.

207. Guérin. Insectes in: Dupperey voyage autour du monte, fait sur la corvette la Coquille. Atas. Paris. 1830.

208. I'erty. M. Delectus animalium articulatorum, quae in itinere per Brasiliam collegerunt J. B. Spix et C. F. de Martius. fol. Monachi. 1830.

209. Curtis. Brit. Ent. 1831. Vol. VIII. a pl. 338-385. (inclusive).

210. Lefierre. A. Description de la P'entatoma Aegyptiaca. Mag. zool. de Guérin. 1831. 1 re $^{r e}$ nn. $n^{\circ}$. 19. р. 20.

211. Lefétre. A Description de Tllalis IIellenica. Mag. zool. de Guérin. 1831. $1^{\text {re }}$ ann. n. 2\%. p. 21.

212. Lefébrre. A. Description de l'Ifalis spinosulu. Mag. zool. de Guérin. 1831. n". 21.

213. Guérin. Mag. zool. I' ann. 1831. n". 8. Description du Tingis dilatala pag. 8. quate identica est 
cum Monunthia erosa Fieber. Ent. Mon. p. 71. t. VI. f. 5-9!) Kolenati.

214. Serville. A. Deseription du genre Peirates de l'ordre des Hémipteres. Annales des Sc. naturelles. 1831.

215. Serville. A. Description du Nématopus elegans. Mém. zool. de Guérin. t. 17. n 27. 1831. - p. 27. Anisoscelis latifolia. 1. 19. p. 18.

216. Suy. Th. Descriptions of new species of Ileteroptervus hémiptère of zorth America. 8". New Harmony Indiana. 1831.

217. Iuhn. C. W. Die Wansenartigen Insecten mit col. Tafeln. 8". I Band. Nürnberg. 1831. a fig. 1-118 (inclusive).

218. Dufour. L. Résumé des Recherches sur les Iemiptères. Annal. des Sc. natur. 1832. $2^{p}$ serie. t. 1. p. 232.

219. Laporte. J. L. Essais d'une classificalion systematique de l'ordre des llémipleres llétéroplères. Magaz. zool. de Guérin. inser. n. 52 à 55.1832.

220. Laporte. J. L. Notice sur un nouveau genre de l'orthe des Homoplères (Ileteronotus). Annal: de I'Soe. Ent. de France. 1832. t. 1. p. 95-98 avec dem. pl. coloriée.

2zi. Laporte. J. L. Mémoire sur quclques nouveaux grenres de l'ordre des Honoptères, aver plo au trait. Amnal. de la Soc. Ent. de France. 1832. t. I. p. 221 i1 231.

222. Prerhemen. A. R. Description at representation du gemre Derbe de Fabricins. Guérin. Magaz. de zoolog. 1832. 11, 36. 
223. Percheron. A. R. Description et représentation du genre Cephalelus. Guérin. Magaz. de Zool. 1832. II. 48 .

22\%. Groy in Griffith's Animal Kingdom. Plilocerus (Reduvini) et Urophor" (Cicadellac) 1832.

225. Schummel. T. E. et Stunnius. I. H. Beiträge zur Entomologie, besonders in Bezug auf Schlesien. II. IIft. 1832. Versuch einer genauen Beschreibung der in Schlesien einheimischen Irten der Familien der Ruderscenzen (Ploteres. Latr.) 80. Breslau. 1832 mit 4 Abbildungen.

226. Brullé. M. Expedition scientifique de Morée. Section des sciences Physiques. Tom. III. I Partie. 3 serie. Zoologie. Paris. 1832. gr. 4. Ilemiplera p. 68 - 80 et pag. 107-113. - Atlas. folio. plant. XXXI. fig. 1-14.

227. Klug. Symbolae physicae. Dec. II. 1832. Eumerus (Reduvini).

L228. Curtis. Entomological Magazine. 1832. Capsini, Tingidites et Cicadellae.

229. Curlis. Brit. Lnt. 1832. Vol. X. a pl. 286 - 433 (inclusive).

230. Hestromd. In Grifith's Animal King dom. XV. 1832. Orthorapha (Membracides).

231. Burmeister. U. Inatbuch der Lntomologie. 1832. Berlin. 8". I. Bd. Allgemeine Entomologie und Entomotomie.

232. Curlis. Brit. Ent. 1833. Vol. X. a pl. $434-181$ (inclusive).

233. Curtis. Entom. Vagazin. 1833. (Itebrus) Ploteres. 23\%. Dufour. L. Recherches anatomingues ol phỵsiologiques sur les Il'́miptères, accompagnèes de consi- 
derations relatives a l'histoire naturelle et à la classilication de ces insectes. Mémoires des Savants étrangers à l'Academie des Sciences. Tirage à part. 1. vol. in $4^{\circ}$. avec 19 pl. Paris. 1833.

235. Dufour. Observation sur une nouvelle espèce d'Anoplius (Ilémiptère) qui n'offre qu'un seul ocelle. Ann. de l'Soc. d. Sc. t. 2. p. 483. 1833.

236. Dufour. Description des trois Hémiptères européens. Aninales lie la Soc. Entom. de France. Tom. III. p. $341-35 \%$ pl. 5. A. B. C. Paris. 1833.

237. Dufour. 1. Mémoire sur les genus Xylocoris, Leptopus et Velia, in Annales de la Socicté Entomologique de France. Tom. II. Paris. 1833. p. 104 - 118. pl. VI. B.

238. Westrrood. P. O. Mémoire sur les genus Xylocoris, If lophila, Hicrophysu, Leptopus, Hicrovelia et IIcbrus. Ann. de la Soc. Ent. de France. Paris. 1833. Tom. III. p. 637-653 pl. VI. fig. 1-6.

239. Guérin. 1. E. Description de 'Anisoscelis alipes. Magaz. zool. 1833. n. 75.

2':0. Bouché. P. Faturgeschichte der sehälichen und nübzlichen Garteninsekten. 8\%. Berlin. 1833.

24. Laporte. M. F. I. Hémiplères nouveanx. Silbermain. Rev. Entom. T. I. $8^{\circ}$. Strassbourg. 1833. p. 35.

292. Germur. L. F. Conspectus generum Cicuduriarum. In Silhermanns Rev. Ent. 'T. I. 1\% 174-18\%. 1833.

ㄴ'13. Burmeister. Nouvelle deassificalion des lnsects. Traduite de l'allemand par G. Silbermann. Revue Ent. 1833. 1. 1. p. 120-126.

2'4. Burmeister. Des Sons que produissent rertains In- 
sectes. In Silbermanns Revue. Ent. T.

I. 1833. p. 161-174.

245. Burmeister. Combophorarum species. Silbermanins Revue Ent. T. I. 1833. p. $227-233$.

246. Burmeister. Mémoire sur la division actuelle des

- Punaises terrestres (Geocores) considérées surtont relativement à la structure dies antennes. Traduit de l'allemand par G. Silbermann. Revue Entomologique. 8". Strassbourg. 183\%. Tom. II. p. $5-26$.

24.7. Germar. Nemoire sur plusieurs especes du genre Cicada Latr. traduites de l'allemand par G. Silbermann. Rev. Ent. 1834. T. II. p. 49-88.

248. Curtis. Brit. Ent. 1834. Vol. XI. a pl. $482-529$ (inclusive).

249. Iahn. C. W. Die Wanzenartigen Insekten mit col. Tafeln. 8". Nürnberg. T. II. 183\%. a fig. 119225 (inclusive).

250. Lexris. 1R. I1. Descriptions of some new genera of British homoptera (avec. fig.). Trans. Soc. Ent. of London. 183\%. t. I. p. 4.

251. Fonscolombe. (E. L. J. N. Boyer de). Notice sur les genres Lithurgus et Phyllocèra. Annal. de la Soc. Ent. de France. 183\%. t. 3. p. 219.

252. Fonscolombe. Description des Kermes qu'on trouve aux environs d'Aix. Annal. de la Soc. Ent. de France. 183\%. t. 3. p. 201.

253. Bouche. Naturgeschichte der Inseliten besonders in Ilinsicht ihrer ersten Zustände als Larven und Puppen. Berlin. 183\%. I. Coceides.

25\%. Klug. Symbolae physicae. Insect. 1834. 
255. Perchéron. In Guérin's Magaz. Zool. 183\%. Cicadellae.

256. Perty. Belectus animalium articulatorum ete. Honachi. 183\%. (Storthia) (Scaptocoris) Pentatomidae.

257. Heyen. F. J. I. Beilräge zur Loologie, gesanmelt auf einer Reise um die Erde. $6^{\text {te }}$ Abtheiluug. Insecten, bearbeitet von W. Erichson und H. Burmeister. Acta Acad. Caes. Leopold. Carol. Nat. Curiosorum. Boiñ. 4". 183\%. Vol. XXI.

258. Burmeister. II. Ilandbuch der Entomologie. Rhynchota. II. Band. 1" Abtheilung. 8". Berlin. 1834. und 1835 .

259. Hamel. J. Ueber Cochenille am Ararat und über Wurzelcochenille im Allgemeineu. In Nímoires de l'Academie Imp. des Scicnces de Saint.-Petersbourg. Sixiemè série. T. I. 4. ${ }^{\circ}$. Sanct-Petersburg. 1835. p. $9-64$.

260. Brandt. J. F. Naturhistorische Bemerkungen über VVurzelcochenill in Vergleich zur Mexicanischen. In Mém. de l'Acad. Imp. des Sc. de St. Pétersbourg. Sixièm. Sér. T. 1. 4". 1835. p. 65-68 mit 2 Kupfertafeln.

261. Curtis. Brit. Ent. 1835. Vol. XII. a pl. 530-577 (inclusive).

262. Westwood. In Zoological Journ. V. 1835. Pentatomides (Deroploa).

263. Uahn die Wanzenartigen Insekten. Nürnberg. 1835. T. III. bis fig. 255.

26\%. Iterich-Schaeffer. G. A. W. Die W'anzenartigen Insekten. Fortsetzung des Ilahn'schen Werkes in 8". Nürnberg. 1835. mit col. Tafeln. T. III. von fig. $256-338$ (incl.). 
265. Herrich-Schaeffer. G. A. W. Nomenclator entomologicus. I. IIT. Regensburg. 1835. kI. 8".

266. Brullé. M. A. et Audouin. M. V. Historie naturelle des Insectrs. Puris. $1835.8^{\circ}$. avec Planches. 6. cahier. Cicadue.

267. Brullé of Audorin. Historie naturelle des Insectes. Petris. 1836. 8, 7. cahier. Notonectae.

268. Curtis. Brit. Ent. 1836. Vol. NII. a pl. 578 - 625 (inclusive).

269. Lesris. in Transactions of the Entomological Society of London. 1836. 1. Vol. Cicadellae.

270. Ecersmunn. E. Catalogus Insectorum Wolgam fluvium inter et montes Uralenses observatorum. Rhynchola. Casani. 1836. In Bull. de la Societé Imperiale des maturalistes de Moscou. 1837. N. 1. p. $33-37$.

271. Costa. Fauna del Regno di Napoli. 1836. Coccides. 272. Curlis. Brit. Ent. 1837. Vol. NV. a pl. 626-673 (inclusive).

273. Curlis. J. Catalogue of Brilish Insects. Edit. II. London. 1837. k1. 80. Hemiptera a p. 217-230.

274. Heyden. (von) Museum Senckenbergianum. 1837. II. Aphidii. (Forda).

275. Hope. Calalugne of Ilemiptera etc. with short latin descriptions of the new species.

276. Westurod. In Trans. of the Entom. Society. London. II. 1837. Hemiptera.

277. Percheron. A. Billiographie Entomologicue. $8^{\circ}$. II. Tom. Londres et Paris. 1837.

278. Kirby. Fanna borealis Americana. 1837. Hemipteru.

279. Germar. E. F. IIemiplere Ileteroptera promontorii bonae spei, collecta a Drege. In Silbermann's Re- 
vue Entomologique. Strassbourg. 1837. Tom. V. p. $121-192$.

280. Krynicki. J. Cicuda Stereni. In Bull. de la Soc. Imp. d. Nat. de Moscou. 1837. N. V. p. $86-88$ tab. VI. lig. 1. b. c. d. e.

281. Lefebrre. In Spinola's Essai sur les IIémiplères hétéroptères. Macroceroea (Lygaeites). 1837.

282. Guérin. M. Fulgor, Nagazin de Zoologie. I'(lris. 1837.

283. Huliduy. In Annal. of the Nat. Hist. I. II. 1838, 1839. Aphidii.

284. Curtis. Brit. Ent. 1838. Vol. XV. a pl. 67t-721 (inclusive).

285. Burmeister. II. Genera Insectorum iconibus illustravit et descripsit. Berolini. 80 ${ }^{\circ}$ 1838. Rhynchota. Vol. I. N. 1 et 2.

286. Guérin-Meneville. F. E. Sur quelques espéses de Cigale. Magazin de Zoologie. Paris. 1838. 8. $t$. IX. tab. 237. 238. pag. 75 -80 .

287. Curtis. Brit. Ent. 1839. Vol. XVI. a pl. 722-769 (inclusive).

288. Burmeister. II. Genera Insectorum. Berolini. 1839. Vol. I. n. 3 et \%. Rhynchota.

289. Spinolte. M. Essai sur les Fulgorelles, sous-tribu de la tribu des Cicudaires, ordre des Rhyngotes. Ann. d. I. Soc. Entomolog. de France. 1839. T. VIII. p. 133-472. pl. $10-17$.

290. White. in Nagazine of Natural History. 1839. IJemiptera.

291. Waga. M. (de Varsovie). Deseription d'une nouvelle espèce d'Ophthalmicus. Ann. d. la Soc. Ent. 
de Franc. 1839. Tom. VIII. p. 523-525. pl. 18. f. 1.1 et 2.

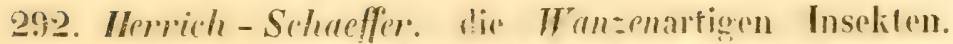
Niirnber\%. 18:39. Tom. IV. a lig. 833!)-8.53 (incl.). 293. Ilerich-Srhueffer. 'Tom. V. 1839) a lig. 45! 5.58 (inclusive).

29\%. Germar. E. Zatsente fir die Entomologie. Leip:ig. 8". 1839. I. B!. 1. Hfr. mil 2 Kuplerlafelu. Beiträge zu einer Moneraphio der Schildwanzen. p. $1-146$.

295. Zelterstedt. J. M. Insecha Lapponica. or. 4". Lipsiae. 18\%. Ilemiplera. p. $257-314$.

296. Trestrond. I. O. Introduction to the mortern Classification of Insects Itemipleza ot IInmoptera. $18 \% 0$.

297. Burmeister. II. Genera Insect. 18\%0. Vol. I. 1. 5 et 6. Rhynchota.

298. Spinola. M. Essai sur les insectes Ilémiplères, Rhyngotes on Hetéroptères. Paris. 8”. 1840.

299. Westrood. In Naturalisté library. I. 18'0. Polyneura (Stridulantes).

300. Rumbur. Faune entomologique de l’Andalonsie. 1840.

301. Hope. Trans. Linn. Soc. London. 1840. X VIII. IIydrocorisiae.

302. Westrood. Magazine of Natural Ilistory. 1840. (Lydda) Fulgorellae.

303. Blanchard. Histoire naturelle des insects (Suites à Bulfon) Lygaeomorphus (Coreides). 1840.

304. Hestrood. J. 0. Arcana Entomologica or Illustrations of new, rare, and interesting Insects. London. gre. 8". 18'1 May. $18 ! 3$ March. Vol. I. a pl. $1-48$. 
305. Westwood. In Annales of Nat. Ilisi. Cistosoma (Stridulantes). 1842.

306. Burmeister. II. Cienera Insect. Vol. I. n. 7. 184. 307. Harlig. Hn Germars Zeilschrift für Ent. 184. Hyponomeutes et Rhizoterus (Aphidii).

308. Coskt In Guerrin's Revue zoologique par la Societé Caviécienne. 1841. Lygaciles et Capsini.

309. Eversmann. E. Acunthia riliata. In Bull. de la Soc. Imp. des Nat, de Moscou. 1841. N. II. pag. 359. tab. VI. f. 6.

310. Burmeisler. II. Genera Inseet. Vol. I. n. 8. 184.

311. Westrrood. J. 0. In Transact. Lim. Soc. London. 1842. XIX. Fulgorellae (Diospolis).

312. Imhoff. L. et Labran. Insekten der Schweiz. Bat sel. kl. 8". 1842. III'se Bündchen. "4 - 60 Ileft.

313. Uerrich-Schaffer. Die Wanzenartigen Insekten. Nïrnberg. 1842. T. VI. a fig. $559-679$.

31\%. IIerrich-Schacffer. 1842. Tom: VII. 1. IIft. a fig. $680-697$.

315. Agassiz. L. Nomenclator zoologicus. Solothurn. 4\% 1843. Fasc. III.

316. Meyer. L. R. Verzeichniss der in der Schweiz einheimischen Rhynghoten. Solothurn. 1843. gr. 8". I. IIft. Capsini. mit $\mathbf{I}$ col. Tafeln.

317. Herrich-Schaeffer. Die Wansenartigen InsektenNiirnberg. 18'3. Tom. VII. 2. IIft. a fig. 698-717.

318. Kaltenbach. J. H. Monographie der Familien der Pflanzenläuse (Phytophthires). Aachen. 8". 1834. mit einer Tafel. Aphidia et Ilyponomeutes.

319. Herrich-Schueffer. Die Wunzenartigen Insekten. 


\section{$-31-$}

Niirnberg. 184t. Tom. VII. 3, 4, 5, 6. IIft. a fig. $718-787$.

320. Imhoff. L. Ins. der Schweiz. Basel. 184\%. 61 74. IIft.

321. Fieber. F. Xav. Entomologisehr Monographien mit 10) Tifeln. Aus den Abhandlungen der k. böhm. Gesellschaft der Wissenschaften. V. Folge. 3. Bd. 4". Prag. 18\%4. Cercopis, Sigara, Tingis, Ophahatmicus.

(Lihri, fuorum annus edit. incognitus).

- Felici. G. B. Lettera intorno al canto delle Cicale. Giornale d'e letterati d'Italia. t. 36. n. 2.

- Risinus. Cur cicada femina non canat. In Th. Simocati. Quest. p. 57.

- Lindenberg. Beschreibung und Abbildung zweier seltenen Surinamischen Laternentrïger. Naturforscher. 13. st. n. 4. p. 19-23. 


\section{$H \mathbb{E} I \mathbb{P} \mathbb{R} \mathbb{R}$ LINNÉI}

\section{RIIVNGOTA FABRIGI.

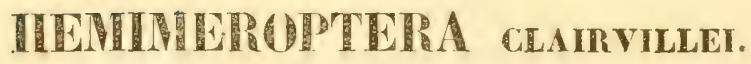

OBSERVATIONES. Meiamorphosis per Nymphas locum mutantes imagini subsimiles, epidermidem pluries exuentes alarumque rudimenta referentes (incompleta secundum Lamark et Burmeister, semicompleta secundum Fabricium); oculi compositi et in imagine etiam simplices (ocelli); antennae articulatae; instrumenta cibaria stizoleicha (pungentia simulque lambentia, non sugentia! $\left.{ }^{*}\right)$, labro coriaceo verticali, mento in rostellum sulcatum producto, setisque tribus loco maxillarum et labii, labello adliuc externo coriaceo, ligula menibranacea el palpis labialibus duabus inferis; prothorax liber; alae quatuor.

*) Hemiptera rotatione spirali simnlque attractiono setarum lateraliumin vagina rostelli circa sctam mediam succum tenuiorem (involulionis Archimedicae instar) evolvunt eumque ligula palpisque momhranaceis lambendo comedunt!. Kolenati. 


\section{O I IS M O I G I A.}

(Signa in tabulis).

\section{A. CAPUT.}

I. Epicranium, facies capitis superior ........... Vide Tab. III et $\mathrm{V}$.

1. Frons, facies epicranii antica " " " " )

2. Tuberculi antenniferi seu stemmata ........ » » III. IV. VI.

3. Antennae.

a. Articulus primus vel basalis .... " » III. IV. V. VI.

b. " secundus... " " " 》 "

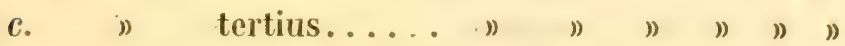

d. " quartus.... " " " " "

e. $)$ quintus vel apicalis.......... " " " "

4. Oculi, (compositi) ..... " " " " " "

5. Regio orbitalis, quae oculos circumdat ....... " 》 " " "

«. Regio supra-orbitalis.. " » III. IV.V.2.VI.

ß. „ prae-orbitalis, in-

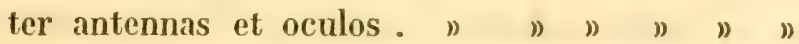

6. Vertex, facies epicranii intermedia et postica, in qua ocelli inveniuntur...... " " III. V.

7. Ocelli, (oculi simplices)... " " " ."

II. IIypostoma, facies capitis inferior ............. » » IV. VI.

$\gamma$. Regio infra-orbitalis sive genae...... " " " "

$\delta$. "retro-orbitalis sive tempora ...., » » »

October. 184:. 
8. Labrum, lamina coriacea, quae instrumenta cibaria externe versus basim tegit. Vide Tab. III. IV. VI.

9. Lamelloe, quae latera rostelli tegunt ......... " " IV. 9.

10. Labellum, lamina angustior coriacea, quae setarum insertionem externe obtegit. " " IV. VI.

11. Rostellum*).

$f$. Articulus basalis vel

"). secundus... " " " " " "

h. $)$ tertius .... " " "

$i$. " quartus vel apicalis ........ " " " "

ع. Seta media (labii locum tenens) ......... " " "

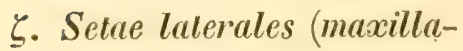
rum locum tenentes) - " " "

$\eta$. Ligula (a labello obtecta) " " " "

ๆ. Palpi labiales (supra a setis, subtus a rostello obtecti)........., " " "

(NB. Ligula et palpi membranaceae in individuis exsiccatis contrahendo valde imminuuntur).

III. Collare, pars capitis postica coarctata, prothoraci inserta aut ab illo obtecta.. ». " IV.

*) Rostellum nil nisi mentum ostendit prolongatum cum lamina involuta, nam rostellum numquam clausum invenitur, semper autem profunde sulcatum aut potius involutum. 


\section{B. PROTHORAX.}

IV. Pronotum, facies prothoracis superior ......... Vide Tab. III et.V.

12. Discus.......... " " " " "

13. Nargo anticus...... " " " " "

14. " lateralis..... " " " " "

k. lateralis anticus ..... » " III. IV. V.

l. " posticus..... " " " " "

15. Margo postïus....... " " " " " "

m. posticus medius.... " " " " "

n. " lateralis ... " " " " "

16. Angulus anticus...... " " " " " "

17. " posticus.... " " " " "

18. " lateralis ... " " " " "

19. „) posticus intermedius (auriculae) ... " " IV.

V. Prosternum, facies prothoracis inferior, media .. " $"$ IV. VI.

VI. Episternum, facies pro-

thoracis inferior, lateralis . " " .

20. Acetabula pedum anteriorum ......... " " n "

21. Stigmata prima in membranosa parte episterni posterioris, quae prothoracem cum mesothorace conjungit ......... » - " " "

22. Insertio vaginae rostelli per membranam ${ }^{*}$ ).... " " ». "

*) Rostelli basis in facie postica intime juncta est per membra. nam musculumque (porrectorem rostelli) cum parte antica prosterni! Kolenati. 


\section{MESOTHORAX.}

VII. Pars antiscutellaris, pars mesothoracis superior antica, quae a prothorace obtegitur........... Vide Tab. III.

23. Dorsulum, pars intermedia......... " " "

24. Pleropega, articulatio hemielytrorum......... (alarum superiorum vel anteriorum)

VIII. Scutellum

o. basis scutelli

p. apex scutelli

$q$. discus scutelli

$r$. margo antica scutelli.

s. " lateralis scutelli

t. Processus scutelli ....

u. Frenum, processus scutelli ad alas posticas decurrens........

IX. Pars postscutellaris vel postscutellum..........

» III.

X. Mesosternum, facies mesothoracis inferior, media : ". $) \quad$ IV. VI.

XI. Scapulae, facies mesothoracis inferior, lateralis...

XII. Acetabula pedum intermediorum ..........

) ) )

D. METATHORAX.

XIII. Metanotum, facies superior metathoracis .... Vide Tab. III. V. 
25. Articulatio tegminum vel alarum inferiorum, posteriorum ......... Vide Tab. III. V.

XIV. Metasternum, facies metathoracis inferior, media " " IV. VI.

XV. Acetabula pedum posteriorum ........... " " " "

XVI. Pleurae, facies metathoracis inferior, lateralis

26. Ostiolum odoriferum, foramen, ex quo succus peculiaris fragrans stillat .

XVII. Opercula tympanica ...

XVIII. Fulcra tympanica ....

\section{E. HEMIELYTRA IIETEROPTERORUI.}

ג. Callus axillaris sive embolyum, pars incrassata articulationis hemielytri cum mesothorace........... Vide Tab. III. IV.

- $\mu$. Corium, facies exterioris hemielytri partis. .

ע. clasus, pars hemielytri interior, scutello proxima ............

$\xi$. Sutura clavi, linea in qua clavus cum corio conjungitur ........

$\pi$. Margo scutellaris clavi, scutellum circumdans............

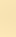

))

))

))

) 
o. Commissura clavi (olim sutura), margo clavi, quae cum opposita collidit. ..........

Vide Tal, III. IV.

o. Angulus clavi, quem sutura et commissura clavi formant

т. Angulus scutellaris clavi, quem margo scutellaris et commissura clavi formant......

v. Appendix sive cuneus sive area apicalis, pars hemielytri apicalis coriacea inter membranam et corium inserta vv. Commissura appendicis vel cunei, in qua cum corio jungitur ..... .

甲. Membrana, pars hemielytri postica hyalina aut diaphana......

$\chi$. Cellulae, a nervis membranae formatae ...

$\psi$. Margo hemielytri externus vel anticus (hemielytro expanso) ...

(w. Maryo hemielytri posticus vel margo corii posticus vel sutura membranae i. e. linea 
in qua corium cum membrana conjungitur Vide Tab. III. IV. w. Margo membranae...

() 》

\section{F. ALAE SUPERIORES IOHOPTERORUM.}

2. Callus axillaris sive embolyum superius...... Vide Tab. V.

$\mu$. Callus axillaris sive embolyum inferius....... " " "

$\nu$. Area axillaris superior et $\nu \nu$. inferior........." " "

$\xi$. Margo anticus vel externus cum radio principali " "

o. Mlargo posticus vel internus " "

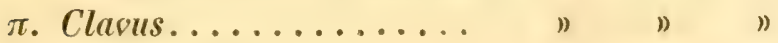

อ. Sulura clavi........ " " "

б. Margo scutellaris clavi.. " "

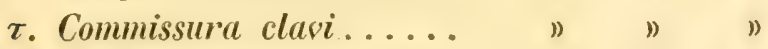

ข. Angulus clavi axillaris.. " " "

$\varphi$. "scutellaris clavi " "

$\chi$. " clavi posticus.. " " "

\%. Nodus sive carpus .... " " " "

$\omega$. Radius internodalis.... " " "

a. Radius medius........ " " "

b. Sector apicalis ....... " " "

c. "nodalis....... " " "

ธ. " subnodalis..... " " "

e. "medius......... " " "

f. "brevis........ " " "

9. Arculus.......... " " "

gg. Claustrum........ " " "

hุ. Parastigma........." " " 


\section{- 40}

i. Cellula sectoris apicalis antica cum anastomosi ${ }_{*}$. Vide Tab. V.

ii. Cellula sectoris apicalis postica..............

f. Cellula sectoris nodalis cum anastomosi ${ }^{+} \ldots . .$, " $^{-}$" "

I. Cellula sectoris subnodalis " " "

m. Intercellula cum anasiomosi $+\ldots \ldots \ldots \ldots$ " " "

n. Cellula sectoris medii... " " "

o. Cellula sectoris bresis .. " " "

p. Cellula radii medii .... " " "

q. Limbus .......... " " "

r. Membranula axillaris vel ponticulus.......... " " "

G. TEGMINA (alae inferiores sive posteriores) HETEROPTERORUM.

f. Area costalis aut marginalis.. Vide Tab. III.

t. " discoidalis ant intermedia " " "

u. " suturalis vel interna.... " " "

v. Callus axillaris ......... " " "

\section{TEGMINA HONOPTERORUM.}

F. Area costalis ............ Vide Tab. V.

t. " intermedia.......... " " "

u. " interna vel suturalis.... " " "

v. Limbus............... " " "

w. Radius principalis........ " " "

x. Claustrum ............ " " "

ท. Radius dichotomus......... " " "

\%. Radius medius........... " " "

v. Radius posticus .......... " " " 
x. Radius brevis........... Vite Tab. V.

y. Radius suturalis.......... " " "

z. Arculus .............. " " "

*. Clasulus.............. " " " "

27. Cellula radii principalis........ " " "

28. Cellula radii dichotomi prima.... " " "

29. Cellula radii dichotomi secunda .. " " "

30. Cellulu radii dichotomi iertiu.... " " "

31. Cellula radii dichotomi quartu... " " "

32. Cellula radii medii.......... " " "

33. Area axilluris superior........ " " "

34. Area axillaris inferior ....... " " "

\section{PEDES.}

K. Pedes anteriores vel antici Vide Tal). III. V. VI.

L. Pedes intermedii...... " " " "

II. Pedes posteriores vel postici........... " " " " "

35. $\operatorname{Coxa} \ldots \ldots \ldots \ldots . "$ " IV. " "

36. Trochanter......" " " "

37. Femur......... " " III. IV. V. VI.

38. Tibia ......... " " " " "

39. Tarsi........ " " " " "

40. Unguis ........ " " " " "

N. ABDONEN. " " " " "

XIX. Facies superior vel dorsum abdominis...... " " III. V.

41. Margo abdominis... " " " " " "

42. Segmentum basale... " " " " " "

43. Ostiola odorifera ..." " III.

44. Spiracula....... " " "

45. Segmentum secundum " " " " " " 
46. Segmentum tertium. Vide Tab. III. IV. V. VI.

47. Segmentmm quarlum. " " " " D

48. Segmentum quintum . " " " " - "

49. Segmentum anale seu podex.......... " " " " "

50. Area segmenti vel discus segmenti...... " " "

51. Margo segmentianticus " " " " "

52. Nargo segmenti posticus.......... " " " " "

53. Angulus segmenti anticus .......... " " " "

54. Angulus segmenti posticus.......... " " " "

\section{Facies abdominis infe-}

rior sive venter....."

55. Carina ventris .....

56. Stigmata ventris ....

57. Iypopygium ........

58. Genitalia .........

\section{HETEROPTERA. \\ Latreille. \\ FRONTIROSTRIA. \\ Zetterstedt.}

Rostellum e capitis antico et supero enascens; hemiclytra coriacea, ad apicem membranacea, se subtegentia, horizontalia; pedes gressorii; ocelli duo; lamella vaginata, oviductus et opercula tympanica desunt. 


\section{Fam. I. HEMEROCORISIAE.}

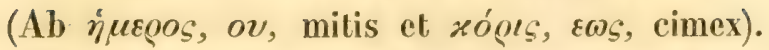

Rostellum acetabula pedum anteriorum excedens, subcylindricum, sublineare, in sulcum pro-et meso-sterni reclinandum.

Degunt in plantis, numquam in aqua, rarius in terra, evocant succum herbis et floribus, spargunt odorem peculiarem coriandrinum.

Omnes hujus familiae sunt:

\section{TESSERACONDYLAE.}

(a $\tau \varepsilon \sigma \sigma \alpha \varrho \varepsilon s$, quatuor et $\kappa o \nu \delta v \lambda \circ$, articuli).

Rostellum articulis quatuor, ut plantis profundius infigi possit.

\section{Tribus I. TESSERATOMIDAE.}

(a $\tau \varepsilon \sigma \sigma u() \varepsilon s$, quatuor et $\tau o \mu i s, i \delta o s$ forceps a $\tau \varepsilon \mu \nu \omega$ scindo).

Antennae articulis quatuor.

I. Antennae clavatae, in epicranio insertae, hemielytra sine appendice.

A. Rostelli articulus tertius quarto brevior.

\section{COREIDES.}

A. Membranae nevi ramosi.

a. Scutellum obtectum.

Genus: PHYLLOMORPII. Laport.

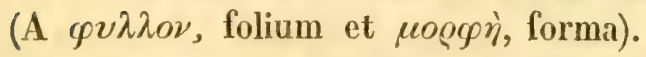

Syromastes. Latr. Coreus. Fabr. Wolff. Germar.

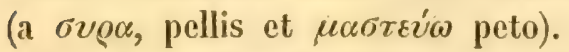

Quam maxime mirabile cimicidum genus, cujus octo jam species Asialicac sive Africanae descriplae etiam duae in Europa meridionali adsunt. 
Charater: Supra excavata, lobis thoracis abdominisque sursum flesis. Exceptis hemielytris et alis omnes corporis partes spinis longis obsitae. Caput paulo longius quam latum, subquadratum oculis ocellisque minutis. In utroque latere rostelli lumellu longa latague. Antennue longitudine superant dimidium corporis, quarum articulus basalis crassus capite longior, secundus brevior, tertius omnium longissimus tenuior, quartus brevissimus crassus et oblengo-ovalis. Spinae symmetrice distributae in antennarum articulo secundo versus apicem et medium, in tertio versus apicem, quarto inermi. Prothoracis lobi laterales extensi, tenues, versus hemielytra conniventes; scutellum obtectum. Hemielytra pellucida. Abdominis segmenta lateralia prolongata, spinosa sursumque llexa. Pedes graciles, tibiis spinosis. Femina differt segmento anali sinuato.

* Abdominis laciniis acutissimis.

Species I. Erinaceus. Herrich-Schaeffer. Wanz. Ins. Bd. IV. pag. 103. tab. 214. fig. 673. Persica. Westwood. Arc. Ent. Tom. I. n. 8, tab. 2. fig. 4.

Pallide testacea aut lurida, albescens, longe spinosissima, laciniis conicis apice acutissimis, antennarum articulo tertio fere duplo longiori secundo, abdominis lobis in medio fusco tinctis.

Long: $4 \frac{1}{4}$ lin. Paris.

Lat. thor. $2^{1 / 2}$, abdom: $3^{1 / 2}$ lin.

IIabitat in desertis aridis, prope Elisabethopolin, Transcaucasiae et confinia P'ersiae, mense Aprili et Majo.

** Abdominis laciniis obtusis.

Species. 2. l'aradoxu. Sparrman. Belirift. Vetensk. Ac. Handl. p. 234-238. 
Paradoxa. Stoll. WV. fig. 10s.

"Fabr. Syst. Rhyng. p. 19\%. n. 14.
Spinola. Ess. Ent. pag. 109.
Westwood. Arc. Ent. Tom. I.
n. 7. tab. 2. fig. 1.

Iutea, fusco - et ochraceo - varia, prothorecis laciniis antice porrectis, abdominis lacinis apice oblique-obtusis et profundius tinctis.

I.ong. 4 lin.

Lat. thor. $2^{1} / 2$, abdom. $2 \frac{3}{4}$ lin.

Inabilat in desertis aridis terrilorii liamabgh et in confinio Persiae, mense Junio.

b. Scutellum distinctum.

* Pronotum postive auriculatum, excisum. Vide Tab. IV. fig. B. 19. m.

\section{Gen. CENTROCORIS. m.}

(A xívt@ov, aculeus et xóøı, cimex).

Character: Supra modice excavata, lobis pronoli abdominisque lateralibus sursum flexis, spinosa. Cap?l Iongius quam latum, fronte protracta, spinosa, oculis exsertis, ocellis modicis. In utroque rostelli latere lamella brevis. Rostellum procurrit ad acetabula pedum intermediorum. Antennae longitudine dimidii corporis, quarum articulus primus spinis brevibus armatus, verrucosus. I'ronoli lobi laterales extensi, crassi, spinosi aut erosi, lobi postici inermes. Scutellum spinosum aut verrucosum, oblongo - triangulare. Illmielytra opaca. Abdomen marginibus aut laciniatis aut profunde sinuatis, renter carinatus. Femora versus apicem incrassata spinosa aut scabra. Mlasculus differt hypopygio acuto.

\section{+ Abdomine laciniato.}


Spec. 3. Westwoodii. mihi.

Vide: Tab. VII. fig. 1.

(In honorem $\mathrm{D}^{i}$. J. Westwood, $\Lambda$ ngliae Entomologi donominata).

Cinnamomea; capite in epicranio triseriatim spinoso, ad Iatera spina una valida antrorsum versa; antennarum articulo primo incrassato, spinis brevibus duplici serie armato, reliquis tenuioribus, verrucosis, ullimo paulo crassiori. Pronoli pars antica depressa, valde verrucosa et spinosa, postica elevata antrorsum tantum spinis pluribus longis armata, ceterum verrucosa; lobi laterales prothoracis incrassati, sursum flexi in spinas quatuor finiti, quarum secunda longior crassiorque. Scutellum verrucis acutis valde elevatis sex, cinnamomeum apice luteo. II $\ell$ miclytra cinnamomea, venis luteis, interstitiis profunde punctato - fossulatis, membrana hyalina. Abdomen cinnamomeum, laciniis lateralibus sursum flexis in spinam exeuntibus pluribusque spinulis armatis. Venter convexus, verrucosus, hirsutus. Femora versus tibias valde incrassata, verrucoso - spinosa, tibiae verrucosae.

Long: 4 lin.

Lat: thor: $1 \frac{3}{4}$, abdom: 2 lin.

Habitat in vepreculis provinciae Transcaucasicae Karabagh et in confinio Persiae, mense Junio.

+ + Abdomine sinuato, antennarum articulo basali inermi.

Spee. 4. Variegata. m.

Vide: Tab. VII. fig. 2.

Nigro - grisea - variegata; epicranio et regione orbitali spinosis, fronte spinis quatuor, retro oculos spinulis, antennarum articulo primo incrassato, triquetro, sulcato, verrucoso, basi nigro, ceterum luteo, secundo 


\section{$-47-$}

atque tertio luteo, quarto breviori, crassiori, nigro. Pronoti pars antica depressa et angustior, postica elevata, verrucosa, margine laterali antico dentato, postico paulo sursum flexo, angulo postico restangulariter exciso, margine postico circa scutellum profunde sinuato, auriculis subacutis, luteis extus obsolete dentatis et leviter sinuatis. Scutellum nigrum, verrucosum, apice luteo. Ilemielytrorum clavus luteus, corium variegatum, verrucosum, membrana ad insertionem maculis duabus nigris, ceterum fusca, nervis nigris. Abdomen nigrum, in medio dorsi luteum, ad latera sursum flexum, sinuatum, venter luteus, leviter verrucosus, obsolete nigro-variegatus. $\mathrm{Fe}$ mora annulis tribus vel quatuor nigro - variegatis, tibiae luteae obsolete - verrucosae.

Long: $5-5 \frac{1}{2}$ lin.

Lat: thor: ant: ${ }^{4} / 5-1$, post: $1^{3 / 5}-1^{4} / \mathrm{s}$, abdom: $2-2^{2} / 5$ lin.

Habitat in Echinope provinciae Elisabethopoleos, communis mense Junio.

+++ Antennarum arliculo primo apice spina una armato. Spec. 5. Pallescens. m. Vide: Tab. VII. fig. 3.

Livida, obsolete ochraceo-variegata. Caput et anfennae testaceae, articulus primus denticulatus apice spina tenui armatus, fronte spinis quatuor ante ocellos, regione pracorbitali et temporali spinulosa; pronotum testaceum, ochraceo - variegatum, verrucosum, margine laterali antico denticulato, postico cum auriculis spinoloso. Scutellum lividum, verrucosum, ad latera denticulatum. IIemielytra ochraceo-variegata, in clavo et commissura maculis albidis. Membrana liusca. Abdominis dorsum pal- 
lidum fasciis marginalibus cimnamomeis, marginibus sursum flexis, renter testaceus, verrucosus. P'edes testacei obsolete verrucosi, tibiae punctis minoribus duobus nigris. -

$$
\begin{aligned}
& \text { Long: } 4 \frac{1}{2} \text { lin. } \\
& \text { Lat: thor: } 1 \frac{1}{2} \text {, abdom: } 1^{3} / 4 \text { lin. }
\end{aligned}
$$

Ilabitat in Carduis provinciae Karabagh, mense Junio. * Pronotum postice leviter sinuatum, absque auriculis.

a. Capul longius ac latius, oculis parsis, parum prominulis.

$\dagger$ Antennarum articulus basalis scaber.

Gen. COREUS. Fabr.

Latr. Lam. Wolff. Fall.

Coriscus. Schrank (a xogiox», puellula). Cimex. Linné.

Character: Antennarum articulus primus crassus, subfalcatus, secundus ceteris longior et tenuior, tertius brevior secundo, quartus paulo brevior tertio, cylindricus, incrassatus.

Rostellum ad acetabula pedum intermediorum porrectum; ocelli modici, pone oculos siti; hemielytra nervis duobus, membrana multis; pedes graciles, femora versus tibias modice sensimque incrassata. Abdominis margines sursum flexi, alas excedentes.

I. Membranae nervis ommibus longitudinaliter ramosis; antennarum arliculo primo triquetro.

1. Thorace obtuso, capite antice spinis tribus porrectis.

A. Antennarum articulo tertio apice alato.

Subgenus: Palethrocoris. m.

(A $\pi \dot{\lambda} \lambda \varepsilon \vartheta \varrho o \nu$, pala et xọ 
Spec. 6. Disciger. m.

Vide: Tab. VII. fig. 4.

Obseure-griseus, subtilissime nigro-punctatus; capitis marginibus luteis; antennae longitudine dimidium corporis excedunt, articulo primo lialcato, triquetro, nigroverrucoso, paulo breviori secundo, secundo omnium longissimo, Juteo, compresso, sublilissime nigro - verrucoso, tertio longitudine primi, basi tenuissimo et luteo, apice palae instar dilatato, alato, compresso, atro, bispino, quarto brevi, cylindrico, nigro. I'rothorax antice coarctatus et depressus, postice elevatus et dilatatus, nigropunctatus, marginibus vix elevatis, luteis, angulis externis rotundis. Scutellum triangulare cum hemielytris nigro-punctatum; membrana obsolete aurea, nervis ramosis parallelis. Abdominis margines hemiely tra paulo excedunt, elevati, nigro-maculati. l'edes graciles, lutei, nigro - verrucosi. Inferior totius insecti facies fulva, nigro-verrucosa.

Long: $5 \frac{1}{2}$ lin.

Lat: palae articuli tertii $2 / 5$ vel $1 / 4$ lin.

Lat: thor: 2 , abdom: $2^{1 / 4}$ lin.

Ilabitat in vepreculis territorii Karabagh, Transcaucasiae, mense Junio, in Arctio.

B. Antennarum articulo secundo et tertio teretibus. Spec. 7. Scapha. Panz. 116. 9.

" Schellenbg. p. 13. tab. 5. fig. 1.

„ Fabr. Syst. Rh. 193. 9.

D $\quad$ Ent. Syst. IV. 127. 2.

"Wolff. II. 69. n. 66. tab. 7. f. 66.

" Curtis. Brit. Egnt. T. IV. pl. 174.

"' Schill. I. 39. 2. tab. 4. f. 2.

" Brullé. Exp. Mor. III. 1. p. 72. n. 18.

Otcober. 1845. 
Scapha. Iiahn. W. J. T. II. p. 103. t. 61. f. 186. "Ilerrich-Schaeffer. Nom. Ent. p. 74 et $4 \mathbf{1}$. Pro-Thorace utrinque rotundato, antennarum articulo secundo rufo, terfio ad basin rufo et subtereli, ad apicem nigro et triquetro, quarto tereti, incrassato, nigro; capite et pronoto albo-marginato; abdomine lato, margine sursum llexo, albo-maculato; ceterum obscure-fuscus.

Long: 6 lin.

Lat: thor: 2, abdom: 3 lin.

Ilabitat in locis herbidis Transcaucasiae et Caucasi.

3. Thorace utrinque unispinoso.

A. Caput inter antennas spina simplici porrecta

A. acula; abdominis medio thorace multo latiore.

*. Antennarum articulo secundo tereli.

Subgenus: VERLUSIA. Spin.

(Ess. Ent. p. 147).

Spec. 8. Quadratus. Fabr. Syst. Rh. 199. 36.

„Ent. Syst. IV. 132. 20.

Wolff. II. 70. n. 67. t. 7. a. ค. 6.

Stoll. If. t. 5. f. 36 . จุ.

Schill. I. 40. n. 3.

Fall. Monog. C. S. 2.

1) Hem. S. 36. 2.

Panz. 118. 12.

Hahn. W. J. II. 104, t. 61. f. 187.

q Rhombeus. Fabr. S. R. 199. 3\%.

Jhhomboideus. Samou. I. 12.

Venator. Don. XI. 41. pl. 375.

Griseo-flavescens, prothorace oblase spinoso, antennarum articulo secundo et tertio rufo, abdomine quadrato.

Long: 5 lin.

Lat: thor: $1 \frac{2}{3}$, abdom: 3 lin. 
Ilabitat rarius in Caucasi et Transcancasiae herbis.

B. Obusu, abdominis medio thorace rix latiore.

Subgen. GONOCERUS. IIerrich - Schacfer.

(A yóvos, ov, soboles et xéqus, cornu).

Spec. 9. Venator. Fabr. Ent. Syst. IV. 128. 4.

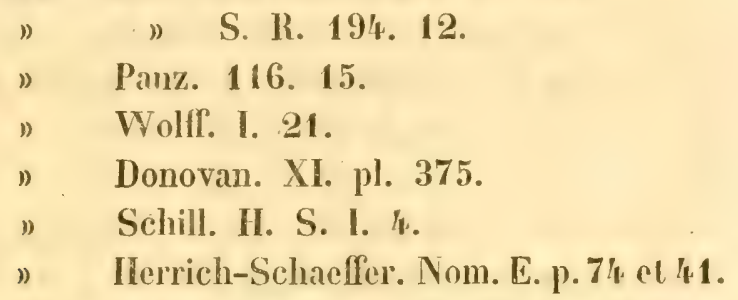

Spina frontali obtusa, abdominis medio thorace vix lattiore, antennarum articulis: secundo, tertio et quarto longitudine decrescentibus, modice clavatis, ferrugineis, apice nigrescentibus. Ceterum cinnamomeus, aut obscure griseus, subtus flavus aut rufus.

Long: $5 \frac{1}{2}-6 \frac{1}{3}$ lin.

Lat: thor: $1^{2} /{ }_{3}-2^{1} / 5$, abdom. 2-2 $2^{1} / 2$ lin.

Var. 10. Juniperi. Dahl. Iferrich-Schaeff. W. J. T. IV. p. 99. t. 141 . f. 445.

Nom. Ent. p. 73.

Minor, supra punctis crebrioribus (ob concentrationem!), subtus flavus.

Habitat in herbis Transcaucasiae et Caucasi.

B. Caput inter antennas spinis duabus acutis, convergentibus.

*. Thoracis angulis porrectis.

Spec. 11. Murginatus. Linné. F. S. 923.

\) N. II. 719. 28.

kossi. F. E. II. 231. n. 1301.
Geoffr. J. I. 446.20.
Scop. Carn. N. 363.


Marginatus. Schaeff. Jc. t. 41. f. 4. 5 .

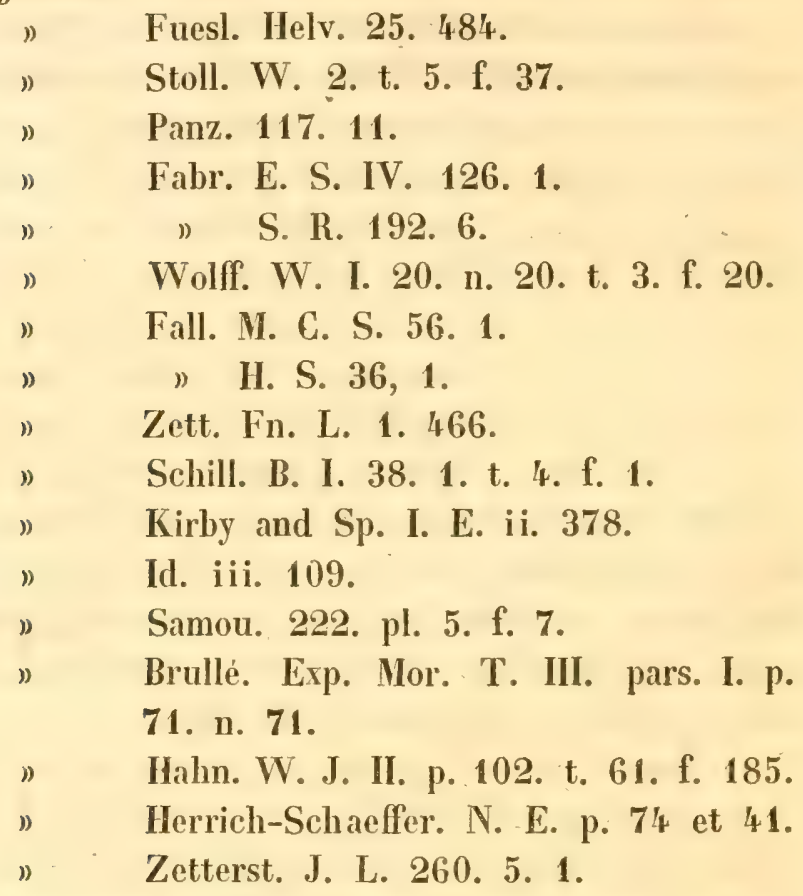

Griseo-brunneus, prothoracis angulis porrectis, subrotundis, membrana aureo-brunnea abdomen paululum superante, articulo antennarum secundo et tertio rufo.

Long: $6-7$ lin.

Lat: thor: $2^{1} / 2^{1}-3^{1 / 5}$, abdom: 3 lin.

Var: Inermis. m.

Spinae duae in capite conniventes desunt, earum loco tantum verrucae.

Habitat communis in Carduis, Rumice et Aretio Caucasi et Transcaucasiae.

II. Membrana pone basin nervo transserso, plurimos nerros longitudinales emittente; antennarum arliculus primus teres, granulatus. 


\section{$-53$}

1. Pronoli margine laterali denticulato.

*. Antennarum arliculus primus, secundus et tertius subcequalis, quarlus crassior.

\section{Subgen. MEROCORIS. IIahn.}

(A unœòs, lumbus et zoors, cimex).

Spec. 12. Denticulatus. Scop. E. C. n. 365. ic. 365.

"Wolff. II. 71. n. 68. t. 7. f. 68.

n Schill. I. 44. n. 8. t. 4. f. 6.

" Hahn. II. p. 106, t. 62. f. 188.

Hirlicornis. Panz. 92. t. 17.

" Fabr. E. S. 17.

1) S. R. 31.

" Coqueb. t. 10. f. 8.

" Fallén. M. C. S. Suppl. 5.

" " I. S. 37. 3.

Scabricornis. Panz. 83. 21.

Herrich-Schaeffer. Nom. E. I. 73 et 42.

Fusco-rufus, subtus ferrugineus; dente medio epicranii modice protracto et una cum thorace et hemielytris punctis confertis prominentibus scabro; antennis hispidis; pronoti margine postico bispinoso; femoribus posticis spinulis sex vel septem, ceterum pedibus hispidis; membrana fusco-nervosa.

Long; /4 lin.

Lat. thor. $1^{2} / 5$, abdom. $1 \frac{1}{2}$ lin.

IIabitat in arenosis et ad radices Artemisiae Cancasi Lhesgici.

2. Pronoti margine laterali inermi, vix crenulato.

\section{Subgen: ATRACTUS. Laport.}

(Ab àtgaxtos fuscus) Arenocoris. Hahn.

(ab arena et xó $\iota \varsigma$. vox hybrida!) 
+ Antennarum articulus tertius quarlusque longitudine acquales.

Spec. 13. Spinipes. Fall. M. C. S. 57. 3.

" $\quad$ H. S. 38. 4.
Panz. 127. 8.
"Schill. I. 43. n. 7. t. 1. f. 13.
Hahn II. p. 110. t. 63. f. 190.
" Herrich-Schaeff. N. E. I. 74 et 42.
Spinol. Ess. Ent. p. 211.

Griseo-brunneus; supra punctulatus; spina capilis media prolongata; pronoti margine paulo crenulato; femoribus posticis apice spinosis.

\section{Long: $4^{2} / 5$ lin.}

Lat: thor: $1 / 2$, abdom: $1 \frac{3}{4}$ lin.

Habitat in Caucaso orientali.

++ Arliculi antennarum, secundus et tertius, graciles, tertius quarto duplo longior.

Spec. 14. Nubilus. Fall. M. C. S. 58. 4.

" II. S. 39. 5.
Ahrens et Germ. F. J. Europ. Fase.
IV. t. 22.
" Schill. I. 47. n. 10. t. 4. f. 5.
" Hahn. II. p. 111. t. 63. f. 191.
" Herrich-Schaeff. N. E. I. 74 et 42.
Spinola. Ess. p. 211.

Cinereo-opacus; spinis capilis aequalibus; pronoto longitudinaliter costato, margine integro; hemiclytris elevato-nervosis; antennarum articulis intermediis rufescentibus, apicali nigro; femoribus edentulis.

Long: 3 lin.

Lat: thor: $3 / 5$, abdom: $1 \frac{1}{5}$ lin.

Habitat in Artemisiis Caucasi et Transcaucasiae. 
$\dagger \dagger$ Antennarum arliculus basalis laevis et cylindricus.

* Caput bifidum; antennue suriegatue, articulus quartus tertio longior, non crassior.

Genus: STENOCEPIALUS. Latr.

Laport. Burm. Ilerrich - Schacffer. Brullé.

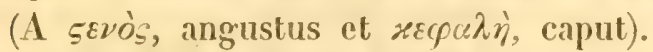

Discranomerus. Ilahn. (a Sixemens, biceps et pui(os; lumbus). Coreus. Fabr. Panz. Schill. Latr. Lygaeus. Fabr. Wolff. Cimex. Linn. Schrank.

Character: Antennae corpore breviores, hispidae, articulo primo longitudine capitis, crassiori; secundo tenuiori et longiori, tertio longitudine primi, quarto longitudine secundi, non hispido. Rostellum ad acetabula pedum intermediorum porrectum, cujus articulus primus longitudine articuli secundi, terlius longitudine quarti. Pronotum triangulare. IIcmiclytra duabus, membrana una cellula basali, e qua in membrana septem aut decem nervi oriuntur. Venter convexus, dorsum ab hemielytris obtectum; pedes longi graciles, tarsorum articulo primo longo. Mas differt abdominis segmento apicali globoso, biverrucato, femina hypoprgio bidentato.

I. Angustior; antennurum arliculus secundus solum apice niger.

Spec. 15. Neglectus. IIerrich-Schaeff. Fortsets. Ifahns. III. p. 55. t. 89 . f. 272 . - Nom. Ent. I. p. 42 et 7 \%.

Griseus; uldominis margine maculato; antennarum articulo secundo in medio non annulato, ultimo rufesecente, versus basim luteo.

Long: 5 lin.

Lat: thor: ${ }^{4} / 5$, abdom: 1 lin. 
IIabitat in locis herbidis prope Elisabethopolin et in Armenia.

II. Latior; antennarum articulus secundus medio et apice niger.

Spec. 16. Nugax. Linn. S. N. ed. Gmelin. 2173. 397.

» Panz. 121. 9.

" Fabr. E. S. IV. 162. 93.

(1) S. R. 200.-42.

"Wolff. I. 30. n. 30. t. 3. f. 30.

" Schill. H. Sil. I. 11. t. 5. f. 2.

"Ilahn. I. p. 22. t. 3. f. 13.

„) Brullé. Exp. Mor. III. 1. 73. 22.

" Herrich-Schaeff. N. E. I. 74 et 42.

1) Spinol. Ess. p. 196.

Agilis. Schrank. II. 1. 82. n. 1125.

" Scopoli. 226. n. 366.

5 punclalus. Goetze. Beitr. II. 278. 23.

1) Stoll. t. 11. f. 79.

Gonymelas. Donov. VII. 5. pl. 218. f. 1.

" Sterv. II. 107.

» Turt. II. 668.

Griseo-opacus; abdominis margine maculato; antennarum articulo sccundo in medio annulato, ultimo nigro, ad basim luteo.

Long: 6 lin.

Lat: thor: et abdom: $1^{3 / 4}$ lin.

Ilabitat in Euphorbiis Transcaucasiae et Caucasi.

* Caput antice bisulcatum; antennis unicoloribus; arliculus quartus terlio bresior, crassiorque.

Genus: RHOPALUS. Schill.

Hahn. Herrich - Schaeff.

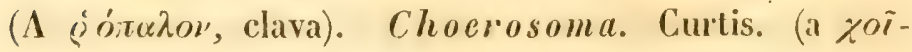


gos, porcus et $\sigma \tilde{\tilde{o}} \mu u$, corpus). Myrmus. Hahn. (a uv́фuos, formica). Corizus. Herrich-Schaeff. Coreus. Fabr. Fall. Latr. Lygaeus. Fab. Wolff. Cimex. Linn.

$\odot$. Linearis; pro-thoracis margo non elevata.

$\odot \odot$. Antennarum articulus primus capite longior; hemielytra abdomine multo breviora. Spec. 17. Arundinis. Curtis. Brit. Ent. VII. pl. 297. Schillingii. Schummel. Schill. Beitr. I. p. 55. ก. 7 .

" Herrich - Schaeff. IV. p. 74. t. 131. f. 402 .

" Herrich-Schaeff. N. E.I.p. 75 et 42. Antennae purpureo-adspersae; color pronoti et hemielytrorum virescens, abdominis supra striis duabus nigris, rentris rufo-luteus, pedum testaceo-virescens.

Long: 7. Lat: $3 / 4$ lin.

Long: abdom. 5 lin.

Habitat in Arundine et locis graminosis Caucasi.

P. Caput latius ac longius; oculis salde prominentibus.

17. Corpus ovale; antennarum articulus primus sequentibus duplo brevior.

Genus: CORIZUS. Fallén.

Dalm. Curtis. Ilahn. Herrich - Schaeffer.

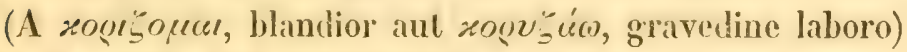
Kleidocerys. Westwood. (a $x \lambda \varepsilon i_{s}, \delta o_{s}^{s}$, clavis et xeques: cornu). Rhopalus. IIerich - Schaeff. Myrmus. Ilahn. Choerosoma. Curtis. Zosmerus. Lap. Coreus. Fabr. Lygaeus. Wolff. Fabr. Cimex. Linné.

Character: Epicranium planum; ocelli pone et retro oculos siti, distincti; pronotum antice angustatum et praeceps, angulis anticis oblusis, posticis subaculis; 
lellum magnum triangulare; hemielytra oblique in corio truncata; saepius subpellucida; femora versus apicem incrassata; corium hemielytrorum nervis duobus apice nonnumquam distichis.

I. Itemielytris opacis coriaceis.

*. Rubro-nigroque varius.

Spec. 18. Hyosciami. Linn. S. N. II. 726. 26.

\begin{tabular}{|c|c|}
\hline & De Geer. J. III. 180. 18. \\
\hline$)$ & Rossi. F. E. 1321. \\
\hline ") & Sulzer. J. t. 11. f. 75 . \\
\hline ") & Panz. F. J. G. 79. 1. t. 19. \\
\hline D & Fabr. E. S. IV. 149. 47. \\
\hline D & " S. R. 218. 63. \\
\hline i) & Schrank. F. boic. II. 1. 80. 1121. \\
\hline " & Wollf. I. 27. n. 27. t. 3. f. 27. \\
\hline " & Fall. H. S. 4h. 6. \\
\hline$D$ & Curtis. Guide. Gen. 112\%. 1. \\
\hline ) & Berk. S. I. 117. \\
\hline ) & Stew. II. 105. \\
\hline ) & Turt. II. 657. \\
\hline ) & Brullé. Exp. M. III. 1. 74. 25. \\
\hline ) & Hahn. I. p. 18. t. 3. f. 10 . \\
\hline ") & $\begin{array}{l}\text { Ilerrich-Schaeffer. N. E. I. p. } \\
\text { et } 42 .\end{array}$ \\
\hline
\end{tabular}

Cimex apterus. Harr. Ex. 91. pl. 26. f. 12.

„) Samou. pl. 5. f. 8.

Pro-Thorax antice niger, postice fascia nigra interrupta in area coccinea; scutclli basis, margo scutellaris clavi et macula corii, antennae pedesque nigri; membrana aeneo - fusca, immaculata.

Long: $4{ }^{1} / 5$ lin.

Lat: thor: $1 \frac{1}{5}$, abdom: $1^{2} / 5$ lin. 
Habitat in Hyosciamo totius Caucasi.

* * Brunneus. Pronoti margo luteus. Spec. 19. Caucasicus. m.

Vide: Tab. VII. fig: 5.

Rufo-brunneus; antennarum articulo tertio rufo; pedibus, margine pronoti et abdominis testaceis.

Caput, pronolum, scutellum et hemielytra rugoso-punctuta, rufo-brunnea, disperse pilosa; antennae dimidii corporis, articulis duobus nigris, tertio rufo, quarto nigro, basi et apice rufescente; pronoti margo lateralis testaceus aut luteus, pone marginem nigrescens; scutellum apice rotundatum, sursum flexum, luteum; hemielytra margine exteruo nigro, membrana hyalina; abdominis dorsum nigrum margine luteo, renter luteus punctis lateralibus et segmento anali nigris. Rostellum luteum, apice nigrum, femora pedum anticorum lutea, intermediorum apice nigrescentia, posticorum in facie superiori pone apicem dimidio nigra; tiliae luteae ad terminos nigrescentes; tarsus primus ad basin luteus ad apicem niger, secundus luteus, tertius niger.

Long: $4^{1 / 5}$ lin.

Lat: thor: et abdom: $1^{2} / \mathrm{s}$ lin.

IIabitat in Asperifoliaceis Caucasi, mense Julio.

II. Memielytris subhyalinis, prothoracis margine con- . colore.

A. Scutclli apex integer; hemielytra abdomen vix superant.

a. Sculclli apex aculus, abdominis dorso maculis tribus margineque luteis.

Spec. 20. Rubescens. m.

Vide: Tab. VII. fig. 6.

Testace-ferrugineus, hispidus; copite, pronolo, scutel- 
lo, articulo antennarum primo et quarto et margine hemielytrorum prope membranam rufis, reliquis viridi-testaceis; scutelli apice sursum flexo; pedilus sparse rufo-maculatis; dorso abdominis nigro, maculis tribus luteis. Long: $2 \frac{4}{5}$ lin.

Lat: thor: $4 / 3$, abdom. 1 lin.

Habitat in Cynancho acuto provinciae Karabagh, Transcaucasiae, mense Junio et Julio.

b. Scutelli apex subacutus.

«. Abdominis segmento anali in dorso punctis duobus testaceis.

$\odot$ Caput cum oculis pronoto antico latius, antennis infuscalis.

Spec: 21. Ericae. Schill. B. I. 4. t. 7. f. 10.

" IIerrich-Schaeff. N. E. I. p. 80 et 46.

(Heterogaster ericae).

Lygaeus Thymi. Fall. H. S. 49. 3.

$\begin{array}{llll}" & \text { " } & \text { Zett. F. L. I. 469. 2. } \\ " & \quad & \text { J } & \text { J. L. p. 262. 8. } 2 .\end{array}$

Griseus; rentre tantum dense piloso; scutelli apice albo aut testaceo; lineola una aut duabus aut margine toto corii postico nigris; membrana prominula; pedibus testaceis subtilissime fusco-punctatis aut brunneis; pronoto antice costula media longitudinali grisea; abdominis dorso nigro.

$$
\begin{aligned}
& \text { Long: } 1^{3} / 5-1^{4} / 5 \text { lin. } \\
& \text { Lat: }{ }^{3} / 5 \text { lin. }
\end{aligned}
$$

Habitat in Caucasi alpibus.

- ๑) Capite cum oculis pronotum anticum vix superante, antennis testaceis.

Var: Graminicola..m. (forsan nova species!)

Pallide luteus; pronoto subtilius ruguloso, linea pone 
costulam in pronoto antico utrinque transversa nigra, impressa, angulis posticis nigris; corio subdiaphano.

Habitat in collibus graminosis provinciae Elisabethopoleos.

3. Abdominis margine parumpunctato, dor'so vitta utrinque serrata nigra.

Spec. 22. Naculatus. Iterrich-Schaeff. VI. p. 2. t. 181. f. 559.

Rufo-ferrugineus, longe pilosus; hemielytris nigropunctatis, membrana prominula; rentre rufo; pedibus rufis brunneo - punctatis.

Long: $3^{1} / 2$ lin.

Lat: 1 lin.

IIabitat in Asclepiadeis Caucasi occidentalis.

c. Scutelli apex rotundatus.

Spec. 23. Crassicornis. Linn. F. S. 952.

\begin{tabular}{|c|c|}
\hline & " S. N. II. 729. 92. \\
\hline & Schaeff. Jc. pl. 13. f. 10. \\
\hline & Panzer. 92. 18. \\
\hline & Fabr. E. S. IV. 168. 114. \\
\hline & " S. R. 201. 46. \\
\hline & $\begin{array}{l}\text { Wolff. IV. 145. n. 140. t. } 14 . \\
\text { f. } 140 .\end{array}$ \\
\hline & Fall. M. C. S. 58. 5. \\
\hline & " II. S. 41. 1. \\
\hline & Zetterst. F. L. I. 467. 1. \\
\hline ) & 》 J. L. 261. 7. 1. \\
\hline & Turt. II. 666. \\
\hline & Schill. I. 50. n. 1. t. 6. f. 2. \\
\hline & Hahn. III. p. 2. t. 73. f. 227. \\
\hline & Herrich-Schaeff. N. E.I. 12.73.74. \\
\hline
\end{tabular}

Griseo-virescens, subpilosus; membrana concolori; $a b$ - 
dominis margine nigro-maculato; femoribus posticis in facie superiori versus apicem nigris.

Long: $3 \frac{1}{5}$ lin.

Lat: $1 \frac{1}{5}$ lin.

Habitat in pratis Transcaucasiae.

B. Scutelli apex obsolete bifidus; hemiclytrorum corii area media pellucida.

a. Abdominis dorso in segmentis duobus ultimis macula longitudinali et utrinque orbiculari nigra. Spec. 24. Magnicornis. Fabr. E. S. IV. 168. 112.

$$
\begin{aligned}
& " \quad \text { S. R. 200. } 44 . \\
& " \quad \text { Fall. N. C. S. 59. } 7 .
\end{aligned}
$$

Pratensis. Fall. H. S. 42. 3.

Parumpunclatus. Panzer. 117. 10.

)

$n$

)

Schill. B. I. 53. 4.

Ilerrich - Schaeffer. N. E. I. 42 et 75 .

Hahn. III. p. 4. t. 7 4. f. 229.

Panzeri. Fieber in Catalogo Programm. Congreg. Medic. Pragae. 1837.

Testaceo - virescens, pilosus; membrana paulo prominula, apice punctis duobus nigris: abdomine luteo, ventre concolori, ad marginem maculis minoribus nigris; pedes lutei nigro-aut brunneo-punctati, femora postica nigro-maculata, tibiae tarsique versus apicem nigrescentes. Long: $3 \frac{1}{5}$ lin.

Lat: $1^{2} / 5$, lin.

Habitat in Asperifoliaceis prope Elisabethopolin Transcaucasiae, mense Junio.

C. Scutelli apex distincte bifidus, luteus. Spec. 25. Capitatus. Fabr. E. S. IV. 169.
)
)
S. R. 201. 49. 
Copiialus. Wolff, II. 75. 72. t. 8. ศ. 72.

Fall. M. C. S. 59. 6.
" II. S. 42. 2.
$"$ Zett. F. L. I. 467. 2.
$" \quad$ J. L. 261. 7. 2.
" $\quad$ Schill. I. 51. n. 2.
"Iahn. III. p. 3. t. 74. f. 228.
"IIerrich-Schaeff. N. E. I. 42 et 74.

Ferrugineo - griseus, pilosus; hemielylris apice opacis rubris; membrana prominula; abdominis margine maculis magnis nigris; tibiis femoribusque rufo-aut brunneoannulatis.

$$
\begin{aligned}
& \text { Long: } 3^{1 / 5} \text { lin. } \\
& \text { Lat: } 1^{2} / 5 \text { lin. }
\end{aligned}
$$

IIabitat in Vincetoxico Georgiae Cancasicac.

b. Corpus lineare; antennurum articulus primus sequentibus non aut vix brevior.

Genus: ALYDUS. Fabr.

Fall. Schill. Kirby. Curtis. Ilahn. IJerrich-Schacffer.

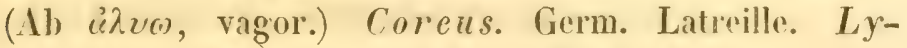
gaeus. Fabr. E. S. Cimex. Linn. De Geer.

Character; Caput magnum, oculis valde prominentibus, collo coarctato; antennue tenues, quarum articulus primus versus apicem paulo incrassatus longiludine secundi sive tertii, quar/us paulo crassior cylindricus subcurvus longiorque; rostellum usque ad acetabula pedum intermediorum porrectum, cujus articulus primus reliquis crassior et longior; ocelli magni el prominentes; pronotum antice depressum, pestice eminens paulocgue lattius; hemielytra cellulis duabus, membranu nervis ramosis; femora postica crassa.

* Tibiae poslicae rectae. 
Spec. 26. Hirsutus. m.

Vide: Tab. VIII. fig. 8.

Niger, hirsutus, punctato-rugosus; capite pronoto angustiori; scutelli apice albo; antennarum articulis nigris, basi luteis, quarto fusco; pedibus nigris, tibiis in medio tarsisque in basi luteis; femoribus posticis paulo incrassatis, quatuor ant quinque dentibus instructis.

\section{Long: $5 \frac{1 / 5}{5}$ lin.}

Lat: $1 \frac{1}{5}$ lin.

IIabitat in Astragalo glycyphyllo et pycnophyllo prope Elisabethopolin et in provincia Karabagh, nec non in provinciis Caspicis.

* Tibiae posticae curvae.

+ in medio incurvatae.

Spec. 27. Lateralis. Germ. Jt. Dalmat. 491.

" " Fr. 8. 21.

» Herrich-Schaeff. V. p. 99. t. 178. f. 549.550 .

» Herrich-Schaeff. N. E. I. 75.

Geranii. Burm. 324.

" Leon-Dufour. p. 2. f. 16.

$\left.\begin{array}{l}\text { Occipes. } \\ \text { Marginatus. Oliv. }\end{array}\right\}$ Berol. Mus.

Brunneus, cinnamomeus aut fusco-rufus; pronoti et hemielylrorum margine laterali tenuissime flavo, puncto medio marginis postici pronoti et apice scutelli llavo-albo; antennarum articulo primo nigro, secundo et tertio flavo in extremis nigro, quarto rufo; femoribus nigris apice incrassatis quinque - dentatis; tibiis llavo-rufis, in extremis nigris, ad tarsos obsolete bidentatis; ventre et dorso rufo. 
Long: 6 lin.

Lat: $1 \frac{1}{3}$ lin.

Var: Antennarum articulo tertio in apice tantum nigro.

Ilabitat in Coronilla varia ef Onobrychide circinata prope Elisabethopolin, Transcaucasiae.

++ Tibiae posticae apice incurratae.

Spec. 28. Tragacanthae. m.

Vide: Tab. VIII. lig. 7.

Niger, punctato-rugosus; antennarum articulo primo nigro, reliquis atro-fuseis, quarto in radice rubescente; capait, pronotum et abdomen pilosum, fronte linea longitudinali rubra, quae paululum in pronotum protenditur; pronoti, hemielytrorum abdominisque margo latus ruber, sculcli apex ruber; membrana prominula fusea; femora postica ad medium valde incrassata, ad extrema decrescentia, clavata, novem - dentala; tibiae ad tarsos nimis curvae, bidentatae, dentibus obtusis.

$$
\begin{aligned}
& \text { Long: } 6^{1 / 4} \text { lin. } \\
& \text { Lat: thor: et abtom: } 1 \frac{1}{3} \text { lin. } \\
& \text { Lat: femorum postic: } 4 / 5 \mathrm{lin} \text {. }
\end{aligned}
$$

Habitat in Astragalo Caucasico (Pall.) etiam Transcaucasiae et in confinio Turciae, prope Alexandrapol mense Septembri et Majo.

c. Corpus crassius quam latum, holosericeum; femora postica securiformia, biseriatim dentata.

\section{Genus: IIEBECERUS. m.}

(Ab $\dot{\eta} \beta \eta$, pubis et жźøฺs cornu).

Character: Corpus crassum inaequale, holosericeum aut dense pilosum; anteniale tenues, quarum articulus primus capite brevior paululum incrassatus, secundus at October. 1845. 
terlius aequales, quartus paulo longior et modice clavatus. Rostellum ad acetabula pedum intermediorum porrectum, cujus articulus primus et tertius paulo incrassatus; cuput angustum; pronotum nimis praeceps; femora postica apice securiformia.

Spec. 29. Persicus. m.

Vide: Tab. VIII. fig. 9.

Nigro-alboque variegatus, holosericeus; capite pronoto antico vix latiori, fronte in spinam obtusam prolongata, rugoso-verrucosa; antennis fuscis, articulo primo rugoso; oculis parvis. Pronotum antice coarctatum mox autem adscendens et postice triplo latius crassiusque, albo-irroratum, margine laterali trisinuato, angulo postico bispino, pone scutellum in auriculam utrinque producte, convexo, inaequali; scutello nigro, spina crassa erecta armato; hemielytra nigra, luteo-alboque variegata, holosericea; membrana nigra. Abdominis margo lutea nigropunctata. Inferior totius insecti facies ob pilos densos albido-holosericea. P'edes fusci, femoribus anticis et intermediis paululum incrassatis obsolete tridentatis, femoribus posticis duplo longioribus ex dimidio in bulbum oblongum valde incrassatis antice flexis, bulbo nigro-alboque-variegato, subtus serie dentium dupla armato, $t i-$ biis in medio annulo obscure testaceo, posticis curvis.

Long: $4 \frac{1}{2}$ lin.

Lat: abdom. $1^{2} / 5$ lin.

Lat: thor: ant: $1 / 2$, postici $1 \frac{1}{2}$ lin.

Crassities thor: postici $1 \frac{1}{2}$ lin; femorum ${ }^{3 / 4}$ lin.

Semel a me captus in Paliuro aculeato vepreculae provinciae Karabagh, in confinio Persiae.

B. Membranae nervi simplices.

a. Fronte costa longitudinali. 


\section{$-67$}

Genus: BERYTUS. Fabr.

(Nomen proprium). Fall. Wolff. Schill. Ilahn.

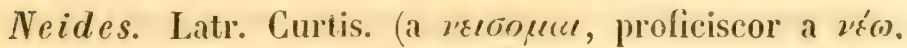
vado). Gerris. Fall. M. Fabr. E. S. Cimex. Frisch. Linn.

Character: Corpus lineare aut filiforme; antennae filiformes, quarum articulus primus longissimus apice clatvatus, secundus brevior et tenuior, quartus brevissimus, cylindricus, incrassatus; rostelli articuli aequales; ocelli minimi; hemielytra nervis tribus longihudinalibus, margine postico obliquo; membrana nervis quinque; pedes tenuissimi, longi, femoribus apice clavatis.

Spec. 30. Caucasicus. m.

Vide: Tab. VIII. fig. 10.

Testaceo-griseus aut rufescens; fironte prolongata; antemnis testaceis, articulo primo nigro; pronotum rufum, profunde punctatum, tricostatum, costis testaceis; hemielytris testaceis, nervis elevatis, in interstitiis profunde nigro-punctatis, apice nigris; membrana fuseo-brunnea; pedibus testaceis.

$$
\begin{aligned}
& \text { Long: } 31 / 5 \text { lin. } \\
& \text { Lat: } 1 / 5 \text { lin. }
\end{aligned}
$$

In arenosis, dumetosis Caucasi et Transcaucasiae.

b. Fronte sulco longiludinali.

\section{Genus: RIIABDOCORIS. m.}

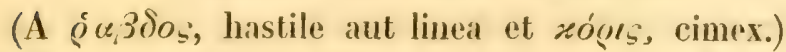

Churacter: Corpus lineare; antennae sicuti pedes longitudine corporis, pedes postici duplo fere longiores quam antici; antennac filiformes, quarum articulus primus apice paulo crassior longitudine quarti, secundus et tertius brevior, quartus falcatus; rostelli articulus quartus brevissimus; hemielytra trinervia cellulisque duabus, nervo cel- 
lulae corii postice in angulo interno processum arcuatum emittente, ita, ut hemielytri margo posticus arcuittus evadat; membrana, nervis undecim parallelis; /emora a latere compressa versus apicem sensim incrassata. Spec. 34. Arcuala. m.

\section{Vide: Tab. VIII. fig. 11.}

Testacea, crebre et profunde punctata; fronte prolongata, apice obtuse bispina; antennurum articulo primo apice nigro, interne testaceo, externo brumeo, reliquis nigris aut fuscis, basi testaceis; pronoto postice dimidio latiore, in medio et al latera lineis impunctatis testaceis, vix elevatis, angulis posticis gibbis, nigris aut fuscis, margine postico tenui, impunctato subrolundato; scutellum profunde punctatum, apice acute spinosum; interstitia hemielytrorum in clavo crebre nigro-punctati, externe in corio tantum pone nervos nigro-punctatat; arcus posticus corii intus nigrescens; membrana hyalina longitudine corii; pedes testacei subtilissime fusco-punctati; venter testaceus.

Long; $7^{1 / 3}$ lin.

Long: antenn: $6{ }^{1} / 2$, femor: post: $3 \frac{1}{4}$, tibiar: post: $31 / 4$ liv.

Lat: thor: 1, abdom: $1 \frac{1}{5}$ lin.

Habitat in foliis Quercus montis Ssalwat, Caucasi Lhesgici, mense Augusto.

II. Antennae in hypostomate insertae, subclavatae, hemielytra absque appendice.

B. Rostelli articulus tertius quarto longior.

\section{Y G A E I E S.}

A. Membrana neris ramosis; ocelli minimi.

Genus: PLATYNOTUS. Schill.

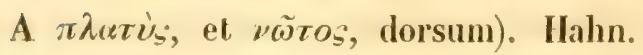


Pyrrhocoris. Dilm. Fall. II. Curtis. (a mejeris, igneus et róołs, cimex.)

Platyocoryza. Fallen. (A $\pi \lambda u \tau v_{\dot{s}}$, latus et sósusce, gravedo).

Megunotus. Laport. (a rígus, $\lambda_{\eta}, u$, magnus et ข๗ัто૬, dorsum).

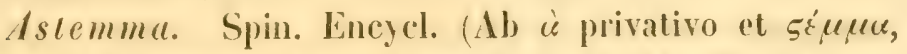
infula.) Lygueus. Fabr. Latr. Fall. M. Wolli. Cimex. Linn. Geoff. De Geer.

Charucter: Corpus ublongo-ovale; antennarum articulus primus crassus, longibuline quarti cylindrici, secundus omnium longissimus, terlius paulo longior primo, hinc longior quarto; hemiclyere binervia, nerro externo in medio corii disticho; deso trapeziformi; membrene nervis ramosis, cellulis tribus; femor a antica paulo crassiora denticulata.

* Embolyum el corium macula nigra.

Spec. 32. Apterus. Linn. S. N. II. 727. n. 78.

1) Rossi. F. E. II. 241. 1322.

" Sulzer K. d. J. tab. 10. f. I4.

» De Geer. III. 276. 20.

") Geoffr. J. I. 4\%0. 11,

) Stoll. II. t. 15. f. 103.

) Fabr. E. S. IV. 161. 90.

" S. R. 227. 116.

" Schrank. F. boic. III. II. I. p. 81. 1123.

" Hausm. in Illig: M. I. 229.

"Wolff. Jc. C. III. 108. n. 102. t.

11. f. 102.

1) Schill. II. S. I. p. 5 I.

') IIahn. I. p. 19. 1. 3. f. 11.

"n Herrich-Sehaeff. N. E. I. p. 75 et 43. 
Apterus. Curtis. Br. E. X. pl. 465.

$" \quad$ "Guide. Gen. 1113. 1.

1) Spinol: Ess. p. 178.

Calmariensis. Fall. H. S. 45. 9. 1.

Ventris nigri segmentorum omnium margo lateralis, penultimi etiam posticus rufus; membrana soepissime incompleta.

Long: $4^{1 / 4}$ lin.

Lat: $13 / 4$ lin.

Communis et frequens in Caucaso et Transcaucasia, in arborum truncis qualemeumque victum praecipue fructus et semina mollia in speluneas colligens. Frequentius in Iberia occurrunt mares membrana evoluta mense Julio et Augusto.

** Corium tantum macula nigra, minori. Spec. 33. Italicus. Rossi. F. E. J. 1523. "Panz. F. J. G. 118. 14. Aegyplius. Linn. S. N. II. 727. 29.

" $\quad$ Mus. Lud. Ulr. 178.

" Fabr. E. S. IV. 115. 69.

" S. R. 222. 87.

" Spinola. Essais. p. 178.

Aegypticus. Hahn. II. p. 10. t. 37. f. 121.

Herrich-Schaeffer. N. E. I. p. 76 et 43.

Venter rufus utrinque serie macularum difformium nigrarum; sexus semper uterque membrana completa.

Long: 4 lin.

Lat: $1 \frac{1}{2} \mathrm{lin}$.

Habitat in Iheria communis cum praecedenti; in provincia Elisabethopoleos jam separatim a praecedenti, inter se tautum communis circa arborum praeprimis sali- 


\section{$-71$}

cum truncos; in provincia Karabagh adhuc frequentior, pracedens autem rarior.

B. Membrana nervis simplicibus; ocelli majores.

a. Caput cum oculis thorace angustius.

a. Feminae segmenta ventralia tria ultima fissa.

+ Femora omnia aeque crassa, i nermia; membrana nervis quatuor, duobus interioribus arcu conjunctis.

Genus: LYGAEUS. Fabr.

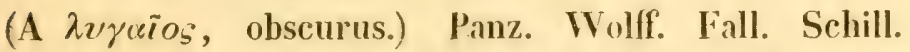
Latr. Ilahn. Herrich-Schaeff. Brullé. Cimex. Linn. De Geer. Geoffr.

Character: Corpus oblongum; capite protracto; antennarum articulo primo quarto breviore; hemielytra binervia, nervo utroque versus apicem disticho, membrana cellula una.

I. Membrana medio immacalata.

1. Capite nigro.

Spec. 34. Familiaris. Fabr. E. S. IV. 119. 48.

") S. R. 219. 6\%.
" Panz. F. G. 79. t. 20?
Herrich - Sehaeff. N. E. I. p. 44
et 76.

Venustus. Bocber. Herrich - Schaeff. N. E. I. p. 58 et 44 .

Membrana basi albida, ceterum nigro - fuseo; pronotum linea media procurrente coccinea; antennue pedesque nigri; hemiclytra coccinea, fascia interupta transversa nigra; scutellum cum clavo nigrum. 
Long: $4^{1} / 2$ lin.

Lat: $1 \frac{1}{2}$ lin.

Habitat in herbidis locis Transcaucasiae.

2. Capite macula bifurca rufa, superficie holosericea. Spec. 35. Saxatilis. Linn. S. N. II. 727. 81.

Rossi. F. E. 1317.

Scopoli. E. C. 371.

Panz. F. G. fase. 79. t. 22.

Schrank. F. b. 538.

Fabr. E. S. IV. 148.46.

) S. R. 212. 62.

Wolff. I. 26. n. 26. t. 3. f. 26.

Schill. I. 59. 2.

Brullé. Exp. Mor. III. 74. 26.

Hahn. II. p. 6. t. 37. f. 119.

Herrich - Schaeff. N. E. I. p. 77. 78. 4.4 .

Membrana fusca; pronotum linea media longitudinali procurrente, caput macula bifurca coccinea; antennis pedibusque nigris; hemielytris coccineis ubique nigro-marginatis, in medio corii macula nigra; scutello nigro lineis coccineis a basi versus discum in angulum convergentibus.

Long: 5 lin.

Lat: $2^{3} / 5$ lin.

Habitat in locis herbidis prope urbem Tillis et Elisabethopol nec non in Caucaso.

3. Capite rufo; pronoti disco scutelloque nigro. Spec. 36. Asiaticus. $\mathrm{m}$.

Vide: Tab...VIII. fig. 12.

Membrana nigro-fusca, apice subacuta; antennae scutellum pedesque nigri; caput coccineum, regione infra- 
et retro-orbitali et circa ocellos nigra: pronoti pars antica medio nigra, macula una coccinea, postica coccinca in medio disci nigro - picta; pro- et episterno nigro macula coccinea; scutellum nigrum; hemielytra coccinea, fascia transversa retrorsum et extrorsum flexa aecuali nigra; margine externo postico et pone membranam nigro, punctis duobus nigris in angulo clavi et lineis ubrinque ad callum axillarem corii divergentilus nigris; renter coccineus maculis aut fasciis transversalibus nigris.

Differt a sequenti thorace quoque longiori.

Long: $6 \frac{1}{2}$ lin.

Lat: 2 lin.

Ilabitat in provincia Karabagh in confinio Persiae.

II. Membrana macula media (nulla apicis) alba.

1. Capitis macula rufa.

* Capilis macula bifurca.

Spec. 37. Militaris. Fabr. E. S. IV. 1/7. 42.

$"$ S. R. 217. 56.
$" \quad$ Germ. Fn. 12. 19.
$" \quad$ Brullé. Exp. Mor. III. 74. 23.
Herrich-Schaeff. N. E. I. p. 44 et 77.

Civilis? Wolff. Jc. C. 25.

Surinamensis? Wollf. lig. 105.

Membrance macula quoque basali alba, apicali rotundata; antennue, sculellum pedesque nigri; caput in regione orbitali et circa ocellos nigrum; pronoti pars antica nigra maculis tribus coccincis, postica pars coccinea, in medio disci nigro-picta; episterno nigro, macula coccinea; hemielytra coccinea, fascia transrersa paululum flexa, aequali, nigra, punctis duobus holosericeis in angulo clavi et lineis utrinque ad callum 
axillarem divergentibus nigris. Venter coccineus maculis vel fasciis transversalibus nigris.

Long: $6^{1} / 2$ lin.

Lat: $2 \frac{1}{4}$ lin.

Habitat in Somchetia, Iberia et Armenia.

* Capitis macula antice rotundata.

Spec. 38. Equestris. Linn. S. N. II. 276. 77.

() De Geer. J. III. 181. 9.

" Geoffr. I. 442. 14.

1) Schaeff. Jc. t. 48. f. 8.

" Elem. 44. 2.

\Panz. F. G. 79. 19.

1) Fabr. E. S. IV. 171. 43.

" $\quad$ S. R. 217. 57.

"Wolff. I. 24. t. 3. f. 24.

"Schill. I. t. 5. f. 4.

) Berk. S. 1. 117.

" Stew. II. 105.

" Turt. II. 656.

"Hahn. 1. p. 21. tab. 3. f. 2f.

" Herrich-Schaeffer. N. E. I. p. 44 et 76.

» Brullé. Exp. Mor. III. 74. 24.

" Curtis. Guide. Gen. 1124. 2.

" ) Br. Ent. X. pl. 481.

Cimex speciosus. Scop. E. C. 127. 369.

" P. Poda. Mus. Graec. p. 59.

Membrana prominula, albo-marginata, maculis duabus lateralibus unaque centrali albis; antennae, pro- et mesothoracis facies inferior pedesque nigri; frontis apice et regione orbitali nigris; pronoti pars antica et postica media nigra, lateralis postica coccinea; scutellum nigrum; 
hemielytra coccinea fascia transversa in medio angustata nigra maculisque duabus in angulo clavi nigris: venter coccineus nigro-maculatus.

Long: $4^{1 / 2}-5^{1} / 2$ lin.

Lat: $1 \frac{1}{2}-1^{3} / 4$ lin.

Frequens in Caucaso et Iberia.

2. Capite immaculato, nigro.

* Membrana maculis duabus basalibus el media alba. Spec. 39. Ventralis. m.

Vide: Tab. IX. fig. 13.

Habitus Platynoti, antennae Lygaei.

Membrana albo-marginata; pronotum coccineum nigro-arcuato-bimaculatum, maculis nimirum oblongis antrorsum fascia transversa sub angulo recto junctis; claro rubro-fusco, corio coccineo macula nigra; renter coccineus margine nigro - punctato, hypopygio nigro; libiae fuscae.

Long: $3^{3} / 4$ lin.

Lat: $1^{2} / 5$ lin.

Habitat in vepreculis provinciae Karabagh.

* Membrana macula media alba.

Spec. 40. Punctum. Geoffr. I. 443. 15.

Panz. F. G. 118. 11.

" Fabr. E. S. IV. 157. 75.

" $)$ S. R. 224.94 .

D Coqueb. Jll. I. 41. t. 10. f. 10.

D Wolff. II. 73. 70. t. 8. f. 70.

" Herrich-Schaeff. N. E. I. p. 44 et 77.

D Brullé. Exp. Mor. III. 74. 27.

Venter niger; pronotum coccineum maculis duabus quadrangularibus nigris, clasus niger, corium coccineum puncto nigro, pedes nigri. 
Long: $2^{1 / 2}$ lin.

Lat: ${ }^{4} / 3$ lin.

Habitat in Caucaso et Iberia, rarus.

III. Membrana macula allera media, altera apicali alla. Spec. 41. P'unclulogullalus. Panz. F. G. 118. 8.

$" \quad$ Fabr: E. S. IV. 77.
$" \quad$ S. R. 224.97.
Herrich - Schaeff. N. E. I.
p. 44 et 77.

Bigullatus. Fabr. E. S. IV. 158. 78.

Ventre in medio rubro; pronoto antice coccineo, postice maculis duabus quadrangularibus nigris; flese coccineo punclis duobus nigris, corio coccineo macula nigra costali, tibiis rufis.

$$
\begin{aligned}
& \text { Long: } 2^{1 / 3} \text { lin. } \\
& \text { Lat: } 2 / 3 \text { lin. }
\end{aligned}
$$

Habitat in Iberia et Armenia, raro.

++ Femora antica crassa, plerumque denlata; membrana nervis quatuor vel quinque solitariis.

\section{Genus: PACIIYMERUS. Lepelletier.}

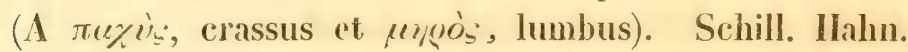
Herrich-Schaeffer. Aphanus. Laporte. Herrich-Schaeff.

(Ab à priv. et quine appareo.). Rhyparochromus.

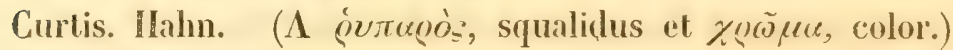
Lygaeus. Fabr. Wolff. Fall. Latr. Cimex. Linn. De Geer. Geoffr.

Charucter: Corpus oblongum; capul protractum; antennarum articulus primus brevis capite numquam longior, secundus omnium longissimus, tertius brevior secundo, quartus paulo crassior tertio; rostellum ad acetabula pedum posticorum porrectum, cujus articulus pri- 
mus crassus longitudine capitis, secundus tenuior ast longior, tertius brevior, quartus tenuissimus al brevissimus; ocelli minimi; hemielylra nervis duobus longiludinalibus fere reclis; membrana nervis dublus evfernis magis flexis, nonnumquam membrana incompleta; libiue anticae paululum flexae.

I. Thoracis latera immarginata.

Spec. 42. Anomalus. m.

Vide: Tab. IX. fig. 14.

Niger, hispidus; hemielytris et thorace postico Perrugineis; cupul, antennate, pronotum anticum, scutelium pedesque nigri, rugosi, hispidi, anguli pronoti externi postici nigri, nilidi; hemichya profunde punctata, lerruginea aut coccinea, margine scutellari clavi et corii postico subtili nigro, in area corii macula nigra; membrena prominens f'usca; venter niger metallescens; femor'a antica inermia; tibiae anticae fuscae.

Long: $3 \frac{2}{3}$ lin.

Lat: thor: ant: $3 / 5$, postic: 1 , abdom: $1 \frac{1}{5}$ lin.

IIabitat in promontorio Araratico Armeniae.

II, Thoracis lateribus marginatis.

1. Membrana immaculata nigra.

* Ater immaculatus.

Spec. 43. Carbonarius. Rossi. F. E. II. 24\%. n. 1330. t. 7. f. 7.

Echii. Panz. F. G. 72. t. 22.

D. Fabr. S. R. 235. 160.

» Schill. I. 73. 13.

1) Hahn. I. p. 137. t. 22. f. 70.

"Herrich - Schaeffer. N. E. I. 78, 79 et p. 44 . 
Aterrimus. Fabr. E. S. Suppl. 541. 96.

" S. R. 229. 124.
$" \quad$ Coqueb. Jll. I. 37. t. 9. f. 10.
Wolff. Jc. C. f. 192.

Ater; femoribus anticis tridentatis; tibiis spinosis; antennarum articulo ultimo fusco-albescenti-piloso.

Long: 4 lin.

Lat: $1 \frac{4}{5}$ lin.

Habitat in Asperifoliaceis Transcaucasiae et Caucasi.

* Griseo-testaceus.

Spec. 44. Pini. Linn. S. N. II. 729. 96.

" " Faun. S. 956.

" De Geer. III. 279. 22. t. 14. f. 22.

"Geoffr. I. \$49. 28.

" Schaeff. Jc. t. 42. f. 12.

1) Fabr. E. S. IV. 163. 97.

" $\quad$ S. R. 229. 125.

W Wolff. II. 74. t. 8. f. 71.

"Zetterst. F. L. I. 470. 5.

" Schill. I. 6\%. n. 1. t. 5. f. 3.

") Stew. II. 106.

D Turt. II. 66\%.

"Hahn. I. p. 38. (absque figura, quae vulgarem refert!)

„Herrich-Schaeff: N. E. I. 44 et 79.

1) Zetterst. J. L. 263. 8. 6.

Niger; pronoti fascia basali hemiclytrisque griseis; corio macula subrhombea nigra; femoribus anticis dentato-serratis.

Long: $3^{3} / 4$ lin.

Lat: $1 \frac{1}{2} \cdot$ lin.

Habitat in sylvis et sub lapidibus Caucasi. 
2. Membrana bicolor.

* Membrana nigra, angulo basali late flavo. Spec. 45. Rolandri. Linn. S. N. II. 729. 98.
)
1) F. S. 957.
„ De Geer. III. 294. 84 .
" Geoffr. I. 459. 51.
.) Schaeff. Jc. t. 87. f. 7.
D Panz. F. G. 118.13.
" Fabr. E. S. IV. 164. 99.
" $)$ S. R. 230.127.
" Wolff. Jc. C. 193.
" Schill. H. S. I. 12.
" Fall. M. C. S. 13.
$»$ H. S. 20.
» Stew. II. 106.
D Turt. II. 664 .
"Steph. S. Cat. 343. 9507.
" Herrich-Schaeff. N. E. I. p. 80 et 44.

Ater, opacus; pronoto subquadrato, aequali (haud depresso); tibiae subspinosae; membrana fusco-nigra, macula rhombea flava.

\section{Long: 3 lin. \\ Lat: 1 lin.}

Habitat in raris Caucasi et Armeniae pinetis.

** Membrana fusca, basi albida.

Spec. 46. Ibericus. $\mathrm{m}$.

Vide: Tab. IX. fig. 15.

Niger; pronoto postico et hemielytris rufis, macula alba in nigredine apicis corii; antennis pedibusque rufovariegatis.

Caput latius thorace anteriori, punctatum, opacum, frontis apice rufo; oculi valde prominuli; pronotum sub- 
planum, punctatum, dimidium anticum nigrum, posticum rufum, imargo antieus subtilissime rufus, color marginis externi postici rufus etiam paululum in anticum procedens; scutellum basi nigrum, apice rufum; hemislyth profund punctata rufi-nervia', margine lato laterali albilo; renter niger metallescens, acelabulu pudum ?rufa; scupulue posticae luteo-marginatac; rostellum rufum, apice nigrum; antennae dimidio corporis I: ngiores, articulis tribus primis rufis, terlio apice fusco, quarlo fusco aut nigro; femora antica rufa dente mo apicali tribusque aut quatuor minoribus basalibus, femor' postice basi rufa, apice fusca; libiae tarsique apice infuscati.

$$
\begin{array}{lll}
\text { Long: } 3 & \text { lin. } \\
\text { Lat: } 2 / 3 & \text { lin. }
\end{array}
$$

Habiat in lberiae dumetis et virgultis, prope urbem Tiflis,

*** Membrana fusca, macula apicali alba.

Spec. 47. Pedestris. Panz. F. G. Fasc. n. 22. 92. 14. Schill. I. 70. n. 10. t. 6. f. 7. Zett. F. L. I. 470 . 4. "J: L. .263. 8. 5 .

Hahn. I. p. 62. t. 10. f. 38.

Herrich - Schaeffer. N. E. I. p. 44 et 79 .

\section{Luscus? Wolff. Jc. C. t. 14. f. 139.}

Niger; pronotum fascia baseos testacea; hemielytre griseo-pallita, macula nigra ante albilum apicem; untennis pedibusque rufo-variegatis, antennarum articulo secundo ferrugineo.

Long: $2 \frac{1}{2}$ lin.

Lat: ${ }^{4} / 3$ lin. 


\section{$-81-$}

IIabitat in vepreculis et virgultis Caucasi et Transcaucasiae.

**** Membrana nigra, margine lacteo.

Spec. 48. Hemipterus. Schill. B. z. E. S. I. 20. t. 6. f. 11. Hahn. I. p. 61. t. 9. f. 37.

1) Ilerrich-Schaeff. N. E. I. p. 78.

Niger, corpore lineari, hemielyeris testaceis, membranae rudimento.

Long: $1 \frac{1}{2}$ lin.

Lat: $2 / s$ lin.

Habitat sub lapidibus ad pagum Kasbek Caucasi centralis.

+ Membrana fusca, maculis inter nerros posticis anteapicalibus pallidis.

Spec. 49. Vulgaris. Schill. I. 65. n. 2. t. 5. f. 8.

„Hahn. I. p. 41. t. 7. f. 25 et 26.

1) Herrich-Schaeff. N. E. I. p. 45 et 80.

Lygaeus pini. Wolff. Jc. C. f. 71.

Tibialis. Megerle in litt:

Niger; pronoti fascia baseos grisea, pallide marginata; hemielytra grisea, macula anguli clavi nigra; tibiis anticis rufis.

Long: $3 \frac{1}{2}$ lin.

Lat: $1 \frac{1}{\mathrm{~s}}$ lin.

Habitat sub lapidibus et foliis delapsis totius Caucasi, deficit in Transcaucasia.

++ Membrana fusca, nervis tenuissime fuscis, albo cinclis.

† Pronolum concolor, hemielytra innotata. Spec. 50. Sylcaticus. Panz. F. G. 120. 6.

Fabr. E. S. IV. 163.98.

" $)$ S. R. 229. 126.

October. 184 :. 
Sylralicus. Fall. M. C. S. 10.

$$
\begin{gathered}
\text { " H. S. } 17 . \\
\text { " Schill. 1. 24. t. 7. f. } 4 . \\
\text { Sylvestris. Hahn. I. 54. t. 9. f. } 33 .
\end{gathered}
$$

Herrich-Schaeff. N. E. I. p. 45 et 80.

Niger; pronoto et hemielytris fuscis; antennis concoloribus pilosis; femoribus inermibus.

$$
\begin{aligned}
& \text { Long: } 2^{1 / 4} \text { lin. } \\
& \text { Lat: } 1 / 2 \text { lin. }
\end{aligned}
$$

Ilabitat juxia agros et sylvas Caucasi et Transeaucasiae.

$\dagger+$ Hemiclytra ante apicem maculata. Spec. 51. Chiragra. Fabr. E. S. IV. 168. 113.

$$
\begin{aligned}
& " \quad \text { Panz. F. G. 122. } 8 . \\
& \downarrow \quad \text { Fabr. S. R. 233. 14\%. } \\
& \text { 1) Fall. M. G. S. 66. } 9 . \\
& » \text { H. S. } 16 . \\
& \text { " Schill. I. 75. 16. t. 6. f. } 9 . \\
& \text { " Hahn. I. p. 56. t. 9. f. 3\%. } \\
& \text { 1) Herrich-Schaeff. N.E. I. p. } 45 \text { et } 78 . \\
& \text { b) Zetterst. J. L. 263. 8. } 10 . \\
& \text { Var: Tibialis. Ilahn. I. p. 23. t. 4. f. } 14 .
\end{aligned}
$$

Niger, pilosus; pars postica pronoti grisea; hemielytra apice nigra et in medio corii punclis duobus in nigredine griseo-pallidis; antennarum articulus secundus basi fuscus aut rufescens; femoribus anticis unidentatis; $t i$ biis tarsisque fuscis aut rufescentibus.

$$
\text { Long: } 2^{1} / 2-3 \text { lin. }
$$

Lat: pronoti et abdom: $3 / 5-1$ lin.

Habitat sub lapidibus et ad arborum truncos Caucasi ot Transcaucasiae. 
+++ Membrana fusco-pallida; nerris marginibusque fuscis.

Spec. 52. Pictus. Panz. F. G. 120. 5.

n) Schill. I. 97. 22. t. 7. f. 1.

" Hahn. I. p. 6\%, t. 10. f. 39.

1) Herrich-Schaeff. N. E. I. p. 45 et 79.

Podagricus. Fall. M. C. S. 70. n. 16.

" H. S. $2 \%$.

Var: Decoratus. Hahn. I. p. 139. t. 22. f. 71. var.

Affinis. Schill. B. I. 80.25.

Niger; pronoti lateribus fasciaque baseos testaceis; hemiclytris testaceis maculis duabus in medio et apice fuscis; antennis pedibusque testaceis.

Var: $\beta$. Fascia pronoti basali obscure-ferruginea, maculis corii apice tantum nigris; femoribus anticis nigris.

Long: $1 \frac{1}{3}$ lin.

Lat: $1 / 3-1 / 2$ lin.

IIabitat sub cortice arborum, sub foliis delapsis in provincia Elisabethopol et Karabagh.

++++ Membrana pellucida, nersis tenuissimis fuscis, albo cinctis, pronoto holosericeo.

* Femoribus inermibus.

Spec. 53. Rusticus. Fall. M. C. S. 70. 17.

») H. S. 6\%, 25.

\Zetterst. F. L. I. 4.70. 3.

$\quad$ Schill. I. 26. t. 7. t. 5 .

IIahn. I. p. 223. t. 36. f. 116.

(Aphanus.)

» Herrich-Schaeff. N. E. I. p. 46 et 80. (Aphanus.)

D Zetterst. J. L. 262. 8. 4. 
Var: Arenarius. IIahn. I. p. 43. t. 7. f. 27.

(Quinquaginta exemplaribus investigatis transgressum rustici in arenarium conspexi. Ceterum color in Pachymeris niger, fuscus et rufus valde fallax ob pinguedinem. Fallén jam varietatem illam descripsit sub $\beta_{0}$, ,membrana albida fusco-striala").

Aler, opacus, impresso-punctahus; hemielytris fuscescentibus; membrana albida; antennis pedibusque rufescentibus aut piceis; antennis nonnumquam tolis fuscis.

Long: $1 \frac{1}{2}$ lin.

Lat: $1 / 2$ lin.

llabilat ad radices plantarum Iberiae.

* Femoribus anticis apice dentato-serratis. Spec. 54. Pedestris. Fall. M. C. S. 71. 18.

(1) H. S. 64. 26.

Sabulosus. Schill. I. 81. 25.

" Hahn. I. p. 22t. t. 36. \&. 117. (Aphunus.)

„ Herrich-Schaeff. N. E. I. 46 et 80. (Aphanus.)

? Si DD. Fallén et Hahn femora antica rite investigarunt, tunc forsan propter femora armata nova Species. (prionoides. mihi.)

Niger, pilosus; thorace holosericeo; hemielytris brunneis, postice fuscis; antennis pedibusque fuscis, tibiis luteis, femoribus anticis ad medium incrassatis angulatis, versus apicem subtilissime dentato - serratis.

Long: 1 lin.

Lat: $2 / \mathrm{s}$ lin.

Halitat sub cortice et foliis delapsis provinciae Karabagh et Armeniae, nec non Iberiae. 
3. Feminae segmenta ventralia bina ultima fissa; corpus depressum, ovatum, antice subacuminatum, membrana nervis quinque solitariis, femora antica crassa, armata.

Genus: PLATYGASTER. Schill.

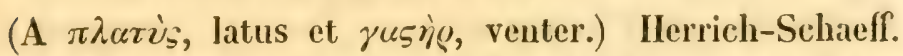
Pachymerus. St. Fargeau. Miris, Lygueus. Fabr. Cimex. Linn.

Character: Antennurum articulus primus crassus, longitudine capitis, secundus tenuis longissimus, apice crassior, tertius brevior secundo, ast longior primo, apice crassior, quartus brevior tertio, ast longior primo; rostellum ad acetabula pedum posticorum porrectum, cujus articulus primus longitudine capitis, secundus longitudine pronoti, tertius longior primo, quartus omnium brevissimus.

* Paulo depressus; antennarum articulo primo capitis apicem longe superante.

Spec. 55. Ferrugineus. Linn. S. N. 733. 99.

Schill. I. 82. 1. t. 7. f. 7.

Herrich - Schaeffer. N. E. I. p.

45 et 80 .

Niger, fascia thoracis baseos hemielytris pedibusque obscure ferrugineis, antennis fuscis, articulo apicali longissimo. Caput impresso-punctatum, opacum; antennis basi apiceque obscurioribus; pronotum antice angustatorotundatum, medio sulcato-depressum, postice dilatatum, angulis posticis tuberculo oblongo; hemielytron cimnitmomeum aut obscure ferrugineum, membranu fusea basi obsolete albida; renter fuscus, medio modice dilatatus, modice convexus, dorso plano; pedes fusci, femoru antica subtus serrata, denticulo uno ceteris majore. 
Long: $2^{2} / 3$ lin.

Lat: 1 lin.

Habitat in Iberiae sylvis sub Strobilaceorum cortice. * * Modice depressus; antennarum articulo primo capitis longitudine.

Spec. 56. Marginatus. $\mathrm{m}$.

Vide: Tab. X. fig. 22.

Rubido-fuscus, profunde grosse punctatus, apterus (absque membrana); capite fronte producto, apice obtuso, antennarum articulo apicali omnium brevissimo; pronoto quadrato, antice paulo angustiori, ad latera cum hemielytris et abdomine luteo marginato; rentre dorsoque cum antennis nigris; trochanteribus tibiisque lividofuscis, femoribus nigris basi apiceque luteis, modice crassis.

$$
\begin{aligned}
& \text { Long: } 2^{4} / 5 \text { lin. } \\
& \text { Lat: thor: } 3 / 5 \text {, abdom: } \mathbf{1}^{2} / 5 \text { lin. }
\end{aligned}
$$

Ilabitat sub lapidibus prope urbem Tillis Iberiae mense jam Februario.

*** Valde depressus; antennarum arliculo primo capitis apicem vix superante.

Spec. 57. Abietis. Linn. S. N. II. 732, 115.

" $\quad$ F. S. 969.

). Mantiss. Ins. II. 307. 290.

Miris abietis. Fabr. E. S. IV. 187. 15.

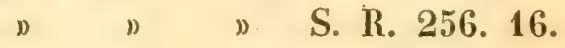
" " Panz. F. G. 92. 22.
( $)$ Schill. I. 83. 2.
» $\quad$ Herrich - Schaeffer. N. E. I. p. 45 et 80 .
" "Berk. S. I. 118.
$»$ \tew. II. 107. 
Miris abietis. Turt. II. 694.

Cimex grossipes. De Geer. Ins. III. 308. 37. t. 15. f. 20. 21.

Pallide cinnamomeus, capite pronoto antico scutelloque nigris; caput cum antennis nigrum, nitens, articulo apicali longitudine tertii; pronotum antice valde coarctatum nigro-nitidum, postice dilatatum luteo-cinnamomeum, margine antico subtilissimo, laterali late luteo; scutellum nigrum; hemielytra cinnamomea, versus clavum et apicem fusco-cinnamomea, crebre punctata; membrana fusco-lutescente, prominente; pedes pallide-rufi, femorc antica nimis crassa bidentata; aldomen rufo-fuscum, deplanatum.

Long: $3^{1} / 5$ lin.

Lat: thor: postici 1 , abdom: $1 \frac{1}{5}$ lin.

Ilabitat sub cortice arborum, pracprimis Pini, in Caucaso.

$\gamma$. Feminae segmenta sentralia quatuor ultima fissa.

1. Abdominis lateribus prominulis.

\section{Genus: IETEROGASTER. Schill.}

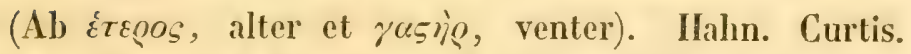
Stenogaster. Ifahm. (a sevos, $\eta$, ov, angustus). Phygas. Fieber. in litt. (a quyùs, $a \delta o_{s}$ u quyn, fuga, fugitivus). Lygaeus. Fabr. Fall.

Character: Forma oblonga; caput protractum; antennae capite cum thorace vix longiores, quarum articulus primus brevis et crassus, secundus omnium longissimus, tertius brevior, quartus longitudine tertii; rostellum ad acetabula pedum intermediorum protractum, articulo primo crasso capitis longitudinem vix attingente, secundo 
longiore et tenuiore, tertio breviore primo, quarlo breviore tertio; ocelli modici; hemielytra nervis duobus longitudinalibus et breviori apicali, intermedio cum lateralibus juncto; membrana nervis quatuor rectis, cellulis tribus, tertia incompleta; femoribus anticis crassioribus.

*. Femoribus inermibus.

+ Antennarum articulo secundo nigro.

Spec. 58. Bicolor. m.

Vide: Tab. IX. fig. 16.

Niger, pronoto postico hemielytrisque griseo- aut riridi - luteis.

Pronotum quadrangulare, antice angustius, angulis subacutis, dimidio antico nigro, punctato-rugoso, griseopiloso, dimidio postico et hemielytris viridi - flavis, profunde nigro-punctatis, nigredine pronoti antici in medium disci postici appendicem acutum formante; scutellum punctato-rugosum, nigrum, apice sursum flexo flavo; membrana albida; venter niger griseo-pilosus, medio rufus, segmentis ante maculam rufam flavo - marginatis, margine externo in dorso nigro-maculato, infra flavo-maculato; antennarum articuli omnes nigri, primo apice, tertio et quarto basi paululum luteis; femoribus nigris apice flavis; tibuis llavis basi et apice nigris; torsis flavis apice infuscatis.

$$
\begin{aligned}
& \text { Long: } 3 \text { lin. } \\
& \text { Lat: } 1 \frac{1}{5} \text { lin. }
\end{aligned}
$$

IIabitat in Labiatis. Transcaucasiac el Caucasi.

++ Antennarum articulo secundo luteo.

- Pronolo concolori.

Spec. 59. Coronillae. m.

Vide: Tal, IX. lig. 17. 
Niger, pronoti margine postico et hemielytris grisenfuscis.

Caput, pronotum et scutellum nigrum, punctato-rugosum; pronotum quadrangulare, antice angustius, angulis subacutis; hemiclytra subtiliter punctata, griseo-pilosa; versus membranam nigrescentia; membrana albida, macula oblonga in medio areae fusca; renter niger griseopilosus, margine externo etiam in dorso flavo-maculato; antennarum articulus primus basi niger, apice luteus, secundus basi paulo niger, ceterum luteus. tertius luteus, basi paululum niger, et annulo lato nigro apicali, quartus basi paululum niger, annulo ante basin luteo, supra dimidium fuscus, apice lutescente; femora antica nigra, apice lutea, reliqua lutea, versus apicem annulo nigro; tibiale luteae; basi nigrae; tursi lutei, apice nigrescentes.

Long: $2 \frac{1}{2}$ lin.

Lat: $/ / 3$ lin.

Habitat in Coronillis Caucasi.

$\odot$ ๑ Pronoto bicolori.

Spec. 60. Walllii. m.

Vide: Tab. IX. fig. 18.

(In honorem Entomologi Bavariae $\mathrm{D}^{i}$. Doctoris Waltl denominatus).

Nigro-griseoque variegatus, profunde-punctalus.

Caput nigrum, regione pracorbitali lutea; pronolum quadrangulare transversum, angulis rotundatis, fuscoflavum, disco nigricante, postice obsolete longitudinaliter nigro-viltatum; scutellum nigrum apice luteo; hemielytra griseo-flavat hine inde obsolete nigro-maculata versus membranam nigrescentia; membrenu allbida; sternum et scapulae nigrare, flavo - limbialae, acefubula luteo-maculata; renter niger ad margines late flavo-maculatus, 
segmento tertio postice rufo-marginato, segmentis tribus posticis rufescentibus; dorsum margine nigro-maculato; antennarum articulus primus ad dinidium niger, apice luteus, secundus basi tantum nigrescens, tertius et quartus niger; femora nigra annulis et maculis inaequalibus luteis; libiae luteae annulis tribus aut maculis fuscis, tarsi lntei. Long: $2^{3 / 5}$ lin.

Lat: 1 lin.

Habitat in Teucriis et Marrubiis Caucasi et Transcaucasiae.

2. Abdominis lateribus et apicemembrana prominula obtectis.

Genus: CYMUS. Hahn.

(A xũue, unda). Eurycephalus. Laport. (ab củ-

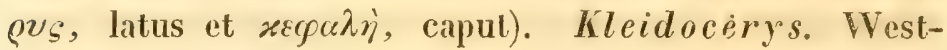
wood. ( $x \lambda \eta i_{s}, 1 \delta o s$, jonic. pro $x \lambda_{\varepsilon} \lambda_{s}$, clavis. ILeterogaster. Schill. Herrich-Schaeff. Lygaeus. Fallén.

Character: Forma oblongo-ovalis; hypopygio subtrilobo, truncato, antennarum articulo primo brevi et crasso, secundo et tertio longitudine fere aequalibus et tenuioribus, quarto cylindrico longiori crassiorique primo; rostellum ad acetabula pedum posticorum porrectum, cujus articulus primus crassus longitudine secundi tenuioris, tertius brevior, quartus omnium brevissimus; hemielytra nervis duobus solitariis paulo flexis; membrana nervis 6 vel septem solitariis, flexis, absque cellulis.

a. Fronte in spinas obtusas binas partita. Spec. 61. Origani. m.

Vide: Tab. X. fig. 19.

Niger; capite, thorace et scutello nigris, punctato-rugosis; pronoto oblongo-quadrangulari, antice angustiori, angulis subacutis, antice toro transverso; hemielytra 
grisea, margine corii postico nigricante; membrana albida; venter niger, parallelus, postice truncatus; antennurum articulus secundus fulvus, basi nigrescens, ceteri nigri; femora nigra, apice fulva, antica unidentata; $t i$ biae fulvae.

Long: $1^{3} / 5$ lin. cum membrana $1^{4} / 5$ lin.

Lat: abdom: $2 / 5$, cum hemielytris $3 / 5$ lin.

IIabitat in Origano vulgari et normali Transcaucasiae et Caucasi.

b. Fronte in spinam subrotundam producta.

a. Antennarum articulo apicali testaceo, fronte bisulcata.

Spec. 62. Saturejae. m.

Vide: Tab. X. fig. 20.

Supra griseo-testaceus, subtus nigrescens; capite testaceo, longitudinaliter bisulcato; pronoto quadrangulari, antice angustiori, testaceo, obscure quinque - costato, punctato, in medio bituberculato, tuberculis nigris; hemielytra maculis binis costalibus, binisque apicalibus; membrana albida; scutello profunde punctato, nigro, margine testaceo; antennis tolis, coxis, pedibus margineque abdominis testaceis.

$$
\begin{aligned}
& \text { Long; } 1 \frac{1}{5} \text { lin. } \\
& \text { Lat: } 2 / 3 \text { lin. }
\end{aligned}
$$

Habitat in Satureja mutica Transcaucasiac.

B. Antennarum articulo apicali nigro.

Spec. 63. Claviculus. Fall. M. C. S. 6\%. 4.

Schill. I. 90, t. 8. f. 6.

Gmr. Faun. 10. 12.

Hahn. 1. p. 77. t. 12. f. 44 .

Herrich-Schaeffer. N. E. I. p. 46 et 80 . 
Lygaeus Caricis. Fallén. H. S. 5.

Cymus glandicolor. var. Hahn. I. p. 79. t.

12. f. 45.

Glandicolor, variolosus; oculis nigris; antennarum articulis tribus testaceis; pronoto oblongo-quadrangulari, scutello et pronoto antico medio costato; hemielytris apice fuscis, margine depressis.

$$
\begin{aligned}
& \text { Long: } 1^{2} \text { lin. } \\
& \text { Lat: } 1 / 2 \text { lin. }
\end{aligned}
$$

Ilabitat ad radices Labiatarum Caucasi.

$\gamma$. Antennis basi apiceque nigris.

Spec. 64. Resedae. Panz. F. G. 40. 20.

1) Schill. I. 89. t. 8. f. 5.

1. Herrich-Schaeff. N. E. I. 46 et 81 .

Lygaeus didymus. Fall. H. S. 50. 4.

" $\quad$ " $\quad$ Zetterst.Act. Holm.1819.71.

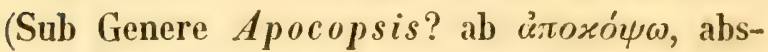
cindo, ni fallor, in uno altero-ve museo occurrit).

Cinnamomeus; hemielytris punctis duobus mediis, quatuor marginis postici fuscis; antennis nigris, articulis intermediis rufis, apice nigrescentibus; abdomen hemielytris membranaque multo brevius; pedes ferruginei; tarsi nigri.

Long: 2, cum membrana $2^{1 / 2}$ lin.

Lat: ${ }^{4} / 5$, cum membrana $1 \frac{1}{5}$ lin.

Inabitat in Cruciferis Georgiae Cancasicae.

c. Fronte in spinam truncatam producta.

Spec. 65. Hyrcanicus, m.

Vide: Tab. X. fig. 21. 
Testace-ferrugincus; scutello bituberculato; antennis pedibusque testaccis; membrana paulo prominula albida.

Caput obscure bituberculatum; fronte bisulcata, testacea; oculis fuscis; ocellis luteis; pronotum transversequadrangulare, punctalo-rugosum, antice angustius, marginibus externis arcuatis, rotundatis, in disco bituberculatum ferrugineum, postice nigricans; scutellum punctato-rugosum, nigrum, basi tuberculis modice elevatis testaceis aut rufescentibus; hemiclytru in corio rufa, clavo testaceo, subpellucido, apice fusco-maculata aut nigrescentia; abdomen testaceum, margine fuseo-maculato; antennarum articulus quartus apice fuscus.

Long: $1 \frac{1}{5}$ lin.

Lat: $3 / 5$ lin.

IIabitat in Teucrio cano et Hyrcanico provinciarum Karabagh et Elisabethopol (Gandscha).

b. Caput cum oculis thorace latius.

Genus; GEOCORIS. Fallén. II.

(A $\gamma^{\prime} \alpha$, terra et xópıs, cimex.) Herrich-Schaeffer.

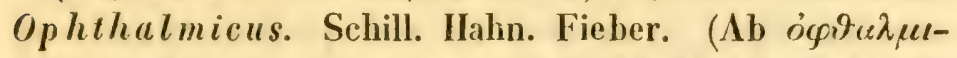
$x_{\dot{5}}$, ocularius). Lygaeus. Fall. M. Salda. Fabr. S. Acanthia. Fabr. E. Cimex. Linn.

Character: Antennae ab oculis remotae, longitudine capitis cum thorace et dimidio scutelli, quarum articulus primus brevis et crassus, secundus tenuior omniumque longissinus, tertius brevior secundo, quartus longitudine tertii, cylindricus et incrassatus; rostellum ad acetabula pedum posticorum porrectum, articulo primo longitudine capitis, secundo breviori, tertio et quarto paulo longiori et tenuiori; hemielytra nervis vix conspiciendis simplicibus; membrana lata prominula, paulo convexa, parallelinervis; trochanteres prominuli; pedes pilosi, femora an- 
tica crassiora et breviora intermediis, postica tenuiora et longiora intermediis; tibiae anticae breves apice crassiores, intermediae longiores anticis, breviores posticis, cum posticis apice non crassiores; hypopygium maris rotundum, feminae fissum cum segmento adjacente. Spec. 66. ô Angularis. Fieber. Ent. Monog. p. 118. n. 8. t. X. f. 1.

\& Siculus. Fieber. Ent. Mon. p. 117. n. 7. t. IX. fig. 27.

Vide: Tab. X. fig. 23.

Claso distincto in sutura mobili; pronoto trapezifor$m i$; niger, profunde-punctatus; pronoto ad angulos posticos luteo; membrana hyalina.

Caput nigrum, antice luteum, hypostomate rufo, fronte sulcata; oculis nigris, rufo-marginatis; antennis nigris, articulo quarto rufo; rostellum nigrum; pronotum trapeziforme nigrum, paulo convexum, antice utrinque callo transversali impunctato, ante callum tenuiter luteo-marginatum vel etiam in medio macula lutea, margine postico late luteo ad angulos externos posticos dilatato in maculas tri-aut quadr-angulares; pro- et epislerno nigro; hemielytra testacea subpellucida, corio pone clavum biseriatim profunde punctato, clavo et margine corii externo serie una punctorum, apice corii obsolete accumulate punctato; abdomen nigrum, nitidum; trochanteres testaceo-albidi; pedes testacei femoribus paulo infuscatis.

Varietas: Scutello toto nigro et pronoto antice non luteo marginato.

Long: $1 \frac{1}{3}-1^{4} / 5$ lin; cum membrana etiam 2 lin. Lat: 1 lin.

Habitat in arenosis vepreculis provinciae Karabagh, Transcaucasiae. 
III. Antennae selaceae, pone oculos insertae, hemielytra cum appendice, ocelli minimi*).

C. Membrana cellulis duabus.

Vide: Tab. IV. E. $v \cdot \chi \cdot \%$.

\section{A P S IN I. Burm. \\ vel}

A S T E M M I T E. Laporte.

(Ab á priv. et sćmece infula).

A. Caput longius ac latius, horizontale; oculis vix prominulis.

Génus: MIIRIS. Fabr.***)

Fall. Zetterst. Latr. Hahn. Wolff. Herrich - Schaeffer.

\section{Curtis. Meyer.}

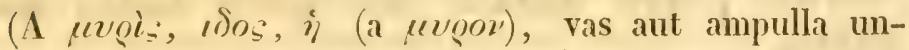
guentaria? verosimilius autem a $\mu i^{\prime} o_{s}$, ov, ó, myrus (piscis) cum dentibus). Stenodema. Laport. (a sevòs, angustus et $\delta \varepsilon ́ \mu a s$, corpus). Capsus. Gmr. fn. Cimex. Linn.

Character: Pronotum horizontale; forma oblonga, linearis; antennae ante oculos in margine epicranii et hypostomatis insertae, longitudine totius fere corporis, articulo primo crasso, curvo, hispido ant rugoso, secundo omnium longissimo, tertio dimidio breviore, tenuiore, quarto tenuissimo; rostellum ad acetabula pedum inter-

*) Sub microscopio pro corporibus opacis constructo ocelli pone et retro oculos conspici possunt. Ocelli in CAPSINIS numquam nigri a me visi; semper enim pellucidi, hinc in capite pallido difficillimi visu. Facilius conspiciendi in lamella epicranii a reliqus capitis partibus separata. Kolenati.

**) Secundum antennas genus Miris ad Coreides pone Rhopalum speclat. Majori jure Systema commutandum esset ita, ut Rhopalus transitum faciat ad Mirides. Kolenati. 


\section{$-96-$}

mediorum porrectum, articulo basali reliquis duplo crassiori apice nodoso; hemielytra nervis duobus ad apicem divergentibus; pedes intermedii brevissimi, postici longissimi.

1. Femora postica apice dentata. Spec. 67. Calcaratus. Fall. M. C. S. 5.

$"$ II. S. 131. 5.
$" \quad$ Zetterst. I. 502. 3.
"Hahn. II. pag. 39.
$" \quad$ Herrich-Schaeff. N. E. I. 47 et 81.
" Meyer. I. 34. 1.
tata. Hahn. I. p. 15. t. 2. f. 8.

Viridis aut flava, etiam fusca; pedibus fusco irroratis, femoribus posticis dentibus ante apicem duobus inacqualibus.

$$
\begin{aligned}
& \text { Long: } 3-3^{3} / 4 \text { lin. } \\
& \text { Lat: } 3 / 5 \text { lin. }
\end{aligned}
$$

In pratis herbidis Caucasi et Transcaucasiae, usque ad alt: 1000 hexapodum.

2. Femora inermia.

a. Femora postica linearia.

a. Antennarum articulo primo thoracis longitudine, longe - piloso.

Spec. 68. Erraticus. Linn. F. S. 961.

" Fall. M. C. S. 111. 6.
$" \quad$ H. S. 132. 6.
" Zett. Adnot. 4.
Hahn. II. p. 78. t. 54. f. 163. $\hat{\circ}$.
164. \&.
Herrich-Schaeffer. N. E. I. p. 47
$\quad$ et 81.
Imhoff. 56.


Erraticus. Meyer. 1. 34. 2.

Quadrilinealus. Schrank. F. b. II. 1. 95.1164.

\& Ilortorum. Wolff. IV.156. 154. t.16. f.154.

lilavo-rufa aut fusea; fronte fere bipartita sursum fle$\mathrm{xa}$, in medio thoracis disci linea viridi elevata, pedibus fusco adspersis.

Long: $3 \frac{3}{4}$ lin.

Lat: 3 lin.

Habitat in graminosis locis Caucasi.

3. Antennarum arliculo primo thorace longiore, hispido.

Spec. 69. Cancusica. m.

Vide: Tab. XI. fig. 25.

Nigro - virescens; capite nigro, fronte producta, apice recta et suncata; untennue corpore longiores, articulo primo nigro, longituline capitis cum thorace, dense hispido, reliquis profunte ferrugineis; rostellum testaceum apice nigro; pronotum nigrum antice rugulis transversis, marginibus lateralibus viridibus; scutellum clavusque hemielytrorum niger; corii apex niger aut fuscus, latera viridia; appendix semper viridis; membrana in medio nigro-fusca, ad latera fusca; abdomen nigrum; femora anlice infra nigra, supra viridi-lutea, fusco-irrorata, apice nigra; intermediu viridi-lutea, fusco-irrorata, apice fusca; postica basi viridi-lutea, fusco-maculata, ceterum nigra; tibiue anticae et intermediae viridi-lutear, fusco-pilosae, basi nigrescentes, poslicae basi nigrae, ceterum fuscae nigro-pilosae; tursi pedum anticorum viridi-fusci, posticorum fusco-nigri.

Long: $3^{3} / 4$ lin.

lat: $3 / 5$ lin.

Habitat in pratis Caucasi el Transcaurasiae.

October. 1845. 
b. Femora postica subclavata.

w. Antennarum articulo primo longe piloso.

+. Antennae corpore parum bresiores, arliculo primo thorace parum breviore. Spec. 70. Laerigatus. Linn. S. N. II. 730. 101.

De Geer. III. 292. 30.

Geoff. I. 452. 26.

Schrank. E. J. A. 558.

Fabr. E. S. IV. 184. 2.

) S. R. 253. 2.

Schrank. F. b. II. 1. 95. 1163.

Wolff. Jc. C. I. f. 36.

Fall. M. C. S. 109. 4.

II. S. 130. 4.

Zetterst. F. J. I. I. 501.

Herrich-Schaeffer. N. E. I. p. 47 et 81 .

Burmeister. HI. II. 265. 1.

Hahn. III. p. 43. t. 85. f. 259.

Zetterst. J. L. 280. 19. 2.

Neyer. I. 35. 3.

Testaceus. Scop. E. C. 135.393.

Virens. Hahn. II. p. 79. t. 54. f. 165.

Viridis, fusca ant luteo-rufa; capite prolongato, fironte tripartita; hemielytrorum margines interni tecti instar conniventes, externi pallidiores; membrana luteo- aut rufo-nervosa; pedibus testaceis fusco-conspersis.

Long: 4 lin.

Lat: $4 / \mathrm{s}$ lin.

Habitat in pratis herbidis Caucasi et Iberiae.

++ Antennarum articulus primus thorace multo brevior. 
Spec. 71. Virens. Linn. S. N. II. 730. 102.

"Fabr. E. S. IV. 185.6.

" "S. R. 25\%. T.

1Wolfi. 2. 78. 75. t. 8, f. 75.

" Ilahn. 111. p. 4.2. \&. 85. f. 257.

" Herrich-Schaeff. N. E. I. p. 47 et 82.

1) Meyer. I. 35. 4.

Laevigatus. Ilahn. II. p. 76. t. 53. f. 161.

Ruficornis. Hahn. II. p. 135. t. 71. f. 220. (absque citato.)

Viridis; fronte prolongata; hemielylris planis, claro tectiformi; membrana viridi-nervis: antennarum apice rufo; pedibus viridibus, vix conspersis.

\section{Long: $3^{3} / 4$ lin.}

$$
\text { lat: } 3 /{ }_{4} \text { lin. }
$$

llabitat in pratis Caucasi.

ß. Antennarum ailiculo primo subnudo.

Spec. 72. Holsatus. Fabr. E. S. IV. 184. '.

$$
\text { ") S. R. 254. } 4 .
$$

" Fall. M. C. S. 7.

1) H. S. 132.7.

"Zetterst. F. J. L. I. 504. 4.

1) Ierrich-Schaeff. N. E. J. p. 47 et 80.

1) Burm. II. II. 265.

" Herrich-Schaeff. III. p. 4.1. t. 85. f. 256 .

1) Meyer. I. 36. 5.

Albidus. Ilahn. II. p. 77. t. 53. f. 162.

Viridi-lutescens ant fusco-lutea; hemielytris extus pallidioribus, fronte protracta apice rotundata, membrana fusco-nervosa, pedes lutei fuseo annulatim conspersi. 


\section{Long: 3 lin. \\ Lat: $3 / 4$ lin.}

Ilabitat in pratis herbidis Cancasi et Iheriae usque ad alt: 1000 hexapodum.

B. Caput latius ac longum, antice declive, oculis prominulis.

1. Thorasis latera acute elevato-marginata.

Genus: LOPUS. Hahn.

\section{Ilerrich - Schaeffer. Meyer.}

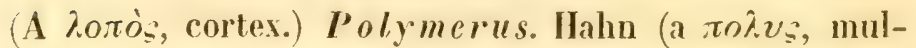
tus et "roos, $80_{s}$, adjumentum, propter appendicem hemielytri; aut a ungò; on femur, per metaplasmum pro uпрor.)

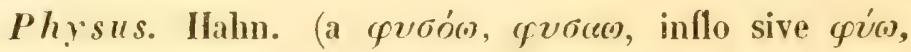
fut. qúo(1), produco, ultimum verosinilius.) Capsus. Fabr. R. Lygaeus. Fab. E. Cimex. Linn. Geoffr. Wolff.

Character: Antennarum articulus primus brevis, paulo crassior, secundus tenuior et longissimus, tertius dimidii secundi, quartus tenuissimus paulo longior primo; rostelli articulus primus crassus et longus, reliqui crassitie et longitudine decrescentes; caput longitudine pronoti dimidii antici; hemielyıra nervis duobus simplicibus rectis, externo et altero medio; membrana cellulis duabus; abdomen cum elytris nimis longum quoad thoracem et caput.

Spec. 73. Gothicus. Linn. S. N. II. 726. 72.

" Mantiss. J. 2. 305. 271.

" Geoffr. I. 445. 19.

D) Panz. F. G.

"Schaeff. Jc. t. 13. f. 5.

Fabr. E. S. IV. 180. 162.

" S. R. 244. 20. 
Gothicus. Wolff. I. 33. t. 4. f. 33.

" Ilahn. 1. p. 12. t. 2. f. 5.

1) Herrich-Schaeff. N. E. I. p. 47 et $8 \%$.

m. İurm. II. 271.

" Meyer. I. 4 1.5.

Cimex superciliosus. Linn. S. N. I. 728. 85.

Capsus fluev-marginatus. Danovan. VII. pl. 245.

Epicranium sine impressione in clypeum transit declive. Ilirtus, niger'; pronoti et elytrorum margine laterali cum appendice miniaceo; pedibus nigris, tibiis in medio rufescentibus.

Long: $2^{3}$, lin.

Lat: 1 lin.

IIabitat in Urticis Caucasi.

2. Thoracis latera immarginata.

A. Pronoti pars antica dimidio angustior postica; antennarum articulus primus capite longior.

a. Pronotum medio antico impressione transversa coarctatum, modice consexum.

Genus: POLYMERUS. IIahn.

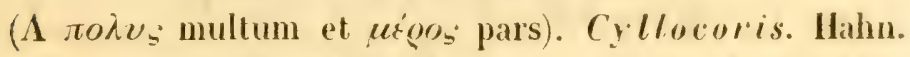
(In discriptionibus est Cyllecoris, in tabulis Cyllocoris. Scheckwanze. Si secundum significationem Scheckwanze formatum est, tunc derivari necquil, nisi a $x \eta \lambda_{1}=$, ¿ $\delta o_{s}$, macula et $x o 0 y_{s}$ et Kelidocoris scribendum esset. Cyllecoris et Cyllocoris Krummwanze derivandum a $x v \lambda \lambda o s, \eta, o \nu$, curvus et rogs; C'illocoris Eselswanze derivandum a $x i \lambda \lambda 0_{-}, 0 u$, asinus el $\left.x o 0\right) s_{5}$.)

Capsus. Herrich - Schaeffer. Phytocoris. HerrichSchaeff. Capsus. Fabr. Fall. Veser. Lygaeus. Fabe. Fall. Cimex. Linn. Gerris. Wolff. 
Character: Forma oblonga, augustata; hemielylo cum abdomine quoad caput et prouotum nimis, plus guam duplo, longiora; oculi a pronoto remoti; pronoti dimidium anticum in forma colli contractum; antennae longitudine insecti, quarum articulus primus crassitic superat secundum, longitudine tertii, secundus apice paulo incrassatus duplo longior primo, quartus tenuissimus; hemielytra nervo unica extrorsum fleso; membrana nerva uno cellulam unam formante; femora internedia anticis, postica intermediis longiora; tibiue longiores femoribus.

a. Nudi.

*. Tibiis inermibus.

Subgenus: KELIUOCORIS. $m$.

+ Scutello albido.

Spec. 74. Histrionicus. Linn. S. N. 728. 89.

Geolfr. 453. 335.

Schrank. En. J. 550.

fierrich-Schaeff. N. E. I. p. 48. et 84 .

" Neyer. I. 90. 75.

Agilis. Fabr. E. S. IV. 182.170.

$"$ " S. R. 247.31.

) Wolff. 4. 150.147, t. 15. f. 147.

Fall. II. C. S. 100.9.

" H. S. 120.10.

DHahn. II. p. 98. t. 60. f. 182.

Atro-rufo-alborque sariegulus, nilidus; pronolu nigro, margine postico late, antico tenuiter flavo; hemielytra membrana fusea: antennurum articulo primo pedibusque ferrugineis.

Var: Antennis totis nigris. 
Long: $3^{4} / \mathrm{s}$ lin.

Lat: $4 / 5$ lin.

Ilabilat in regione dumetosa Caucasi usque ad 700 hexapod.

++ . Scutello nigro.

Spec. 75. Fluromaculatus. Panz. F. G. 92. 16.

Fabr. E. S. IV. 182.169.

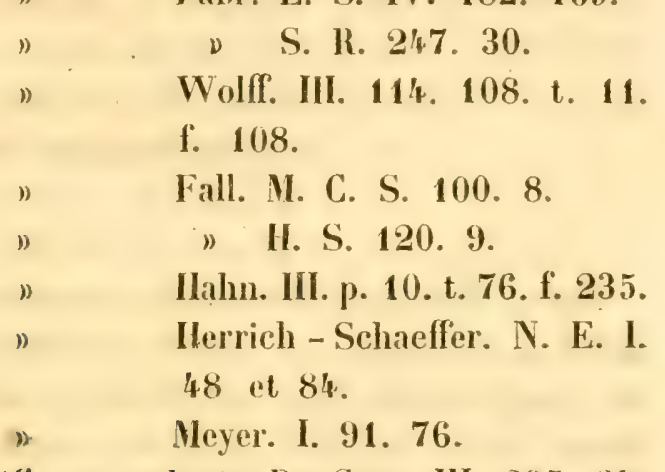

Quadriflaromaculatis. De Geer. III. 295. 34.

Niger, opacus; hemielytris maculis duabus flavis, apice nigris; antennarum articulo primo, pedibusque ferrugineis.

Long: 2 lin.

Lat: $3 / 5$ lin.

Ilabitat in pratis provinciae Karabigh, transgressum. format in distiguendum. Ierrich-Schaeffer.

** Tibiae spinis brevibus.

Subgenus: CYLLOCORIS. Hahn.

+. Antennis variegatis.

Spec. 76. Striatus. Linn. S. N. II. 730. 105.

" Faen. Sv. 960.

Petiver. Gazoph. N. el A. 1. 26. f. 1. De Geer. III. 290. 29. 1. 15. f. 14. 15.

Sulzer. Abg. Gesch. to 10. f. 15.

Geoffr. I. 45\%. 38. 
Striulus. Schaeff. Jc. t. 13. f. 4.

" Panzer. F. G. 93. 22.

1) Fabr. E. S. IV. 186.

" $"$ S. R. 25.5. 15.

1) Schrank. F. b. II. 1. 90. 114T.

"Wolff. 37. 1. 4. f. 37.

" Fall. I. C. S. 37.

" $\quad$ II. S. 14.

v Hahn. II. p. 13\%. t. 71. f. 219.

" Herrich-Schaeff, N. E. I. 48 et $\mathbf{8 7}$.

n Imhoff. 58.

n Neyer. I. 94. 80.

Nigro, ferrugineo testuceoque variequtus, striutus: pronoti basi et medio disci luteis; scutello luteo, lineola media longitudinali basi lateribusque nigris; hemielytris fuscis, flavo striatis, appendice aurantiaco; membruna fusca: antennarum articulo primo rufo, apice summo nigro, secundo nigro-fusco, basi rufo, terlio basi luteo aut albido ceterum rufo et fusco; pedibus ferrugineis.

Iong: $4 \frac{1}{2}$ lin.

Lat: $\mathbf{I}^{1}$ lin.

Habitat in fruticibus Caucasi.

++ . Antennis nigris.

Spec. 77. Caricis. Fall. N. C. S. 102. 14.

\) H. S. 123.15.

Hahn. II. p. 100, t. 60. f. 184.

Herrich-Schaeff. N. E. I. 52 et 83

Neyer: I. 85. 66.

Plures transgressus format in:

Ambulantem. Fall: II. C. S. 18.

" " H. S. 20.

"Zetterst. Ins. Lapp. 7. 
Ambulantem. Herrich-Schateff. III. p. 109. t. 108. f. 335-337.

" $\quad$ Neyer. I. 86. 7. 6. I. P. 82.

Rufifions. Fall. M. C. S. 105. 19.

Niger, pedibus flavis; hemielytris in mere hyalinis, puncto costali ad basin et apicem appendicis fusco aut nigro, in femina luteo - fuscis prater cunci nigredinem apicalem et basalem nervo medio et externo corii fusconigro; antennarum apice fusco.

$$
\begin{aligned}
& \text { Long: } 2 \text { lin. } \\
& \text { Lat: } 1 / 2 \text { lin. }
\end{aligned}
$$

Habitat in locis graminosis Caucasi.

+++ Antennis rufis.

Spec. 78. Luridus. Fall. M. C. S. 94. 78.

$$
\begin{aligned}
& \text { ». H. S. 112. } 69 . \\
& \text { "Herrich-Schaeff. III. p. 87. t. } 101 . \\
& \text { f. } 312 . \\
& \text { 1) }>\quad \text { N. E. I. } 53 \text { et } 85 . \\
& \text { 1) Meyer. I. 109. } 101 .
\end{aligned}
$$

Testuceus aut kermesinus subnilidus; antennis ferrugineis; pedibus testaceis; hemielytris testaceis, hyalinis.

$$
\begin{aligned}
& \text { Long: } 1 \frac{1}{2} \text { lin. } \\
& \text { Lat: } 1 / 2 \mathrm{lin} \text {. }
\end{aligned}
$$

Habitat in locis graminosis Caucasi.

B. Subpubescentes.

\section{Subgenus: LOPIIYRUS. m.}

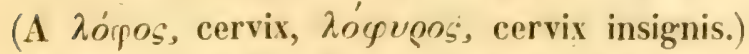

*. Capite lineola frontali lutea, pronoto longiori ac lato.

Spec. 79. Neyeri. m.

Vide: Tab. XI. fig. 26. 
(In honorem $\mathrm{D}^{i}$. L. R. Neyer, Ilemipterologi Ilelvetiae, qui Capsinorum monographiam edidit, denominata.)

Nigra; supra viridi-llava vel sulphurea et nigro-picta. Caput nigrum nitidum, lineola tenuissima longitudinali lutea, pronotum antice in collum coarctalum, capite angustius, impressionibus semilunaribus, postice vix dimidio latius, sulphureum, vittis duabus longitudinalibus nigris, conicis, quarum basis usque ad marginem posticum tendit, apex autem coarctatus iterumene in maculam orbicularem dilatatus marginem anticum non allingit; episternum sulphureum, maculis duabus nigris; scutellum sulphureum, basi nigrum; hemielytra cum appendice sulphurea, clavo, maculaque in medio corii triangulari nigris, triangulo apice acuto antrorsum verso; membratne nigro-fusea; antennae pedesque rufo-fusei aut nigri; venter niger, segmentis nonnumquam subtilissime luteo marginatis.

Long: $3^{3} / 4$ lin.

Lat: $1 \frac{1}{s}$ lin.

Formosissima Capsinorum species habitat in Umbelliferis prope pagum Tschaikaent ad fluvium Kjuraktschai territorii Airum provinciae Karabagh Transcaucasice et in pratis subalpinis montium ejusdem territorii Kaepeset Muroff - Dagh.

* *apite lineolis supraorbitalibus luteis, pronoto aeque longo ac lato.

Spec. 80. Sexgututus. Fabr. E. S. IV. 174.139.

" $\quad$ S. R. 237.172.
$" \quad$ Fall. M. C. S. 43.
" II. S. 86.20.
IIerrich-Schaelf. N. E. 1.48 el 87.




\section{$-107$}

Sexgutlatus. Ilerrich-Schaeff. III. p. 77. I. 98. f. 295.

" $\quad$ Meyer. 1. 92. 77.

Niger ; supro aurantiaco-luteus et nigro-fuscoque pictus.

Caput nigrum, nitidum, regionibus orbitalibus luteis; pronotum antice anguslius, postice dimidio latius, auranliacum, vitlis duabus longitudinalibus conicis nigris, quarum basis usque arl marginem posticum tendit, apes in maculam semi-orbicularem in alteram confluentem marginem anticum non attingit; episternum nigrum; scutellum aurantiacum, basi nigrum; hemielylra aurantiacolutea, clavo basi luteo ceterum nigro-fusco, corio macula media obsolete triangulari alteraque externa minori nigro-fusca, appendicis apice nigro-fusco; membranu fusco-pallida, cellula fusea; antennae fuscae, pedes pallidefusci aut fusco-testacei; venter niger, segmentis tenuissime aurantiaco-marginatis.

$$
\begin{aligned}
& \text { Long: } 3^{1 / 2} \text { lin. } \\
& \text { Lat: } 1 \text { lin. }
\end{aligned}
$$

Ilabitat in pratis subalpinis Caucasi.

$\gamma$. Inemielytri margine externo ciliato.

Sulgenus: BLEPIIARIDOPTERUS. m.

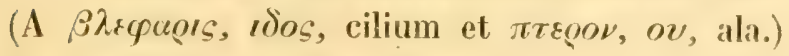

*. Luridus, pronoto gibboso.

Spec. 81. Collaris, Fallén. II. C. S. 103. 18.
" $\quad$ II. S. 125. 19.
II Ilahn. II. p. 120. t. 66. f. 203.
1) Herrich-Schaeff. N. E. I. 48 et 83 .
1) Meyer. I. 83. 63.

Gerris errans. Wolff. Jc. C. lig. 155.

Thorace angustato, antice gibbo dupplicato; hemicly- 


\section{$-108-$}

tris luridis, fusco-punctatis nervis nigrescentibus et matculatis, appendice postice nigro; femoribus nigro- aut fusco-maculatis.

$$
\begin{aligned}
& \text { Long: } 2 \text { lin. } \\
& \text { Lat: } 1 / 2 \text { lin. }
\end{aligned}
$$

llabitat in pratis Caucasi et Transcaucasiae.

** Viridi-nilens, pronoto transcersim sulcato. Spec. 82. Angulatus. Fall. M. C. S. 76. 32..

$$
\begin{aligned}
& " \text { " II. S. } 8 . \\
& " \text { Zetterst. F. J. L. I. p. 487. I. } 5 . \\
& " \quad \text { J. L. p. 272. 11. 6. }
\end{aligned}
$$

Viridis, elongatus, taeris, pilis nigris ad latera corii obsitus; pronoti angulis posticis apice nigris; antennarum articulo primo apice albido; membrana hyalina, nervo aeruginoso.

Long: $2-2^{1} / \mathrm{s}$ lin.

Lat: $1 / 2$ lin.

Ilabitat in Betulis, Loniceris et Salicibus Caucasi alpium, rarus.

***. Viridis, opacus, pronoto salde declisi ad marginem anticum coarctato.

Spec. 83. Bimaculatus. Holfsgg. Herrich-Schaeff. N. E. I. 51 et 82 .

(Nus. Berol.)

" $"$ Herrich-Schaeff. VI. p. 48. t. 169. f. 607.

Viridis nigro-pilosus; pronoti maculis binis mediis singulaque humerali, scutello lineola, hemielytris strigis obsoletis, apprendicis apice nigris; membrana fusco-maculata; pedibus antennisque viridi-ferrugineis, antennarum articulorum apicibus fusco-nigris. 


\section{$-109$}

Long: $3^{1 / 2}$ lin.

Lat: 1 lin.

Ilabitat in Celtide caucasica provinciae Elisahethopol.

B. Antennarum arliculus primus capite rix longior.

b. Pronotum consexum, impressione in medio antico lesi, antennarum articulo secundo apice incrassato.

Genus: PIIYTOCORIS. Fallén.

(A gutòn, planta et zoois, cimex.) Hahn. HerrichSchaeff. Poecilosoma. Steph. (a torxihos, variegatus

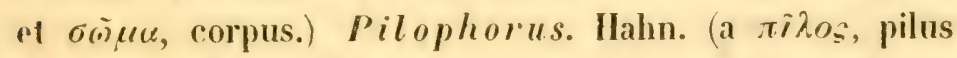
et qouta, fero.) Lygus. Hahn. Polymerus. Ifahn. Capsus. Fabr. Hahn. Herrich-Schaeff. Neyer. Lygaeus. Fabr. Cimex. De Geer.

Character: Forma oblongo-ovalis; hemielytra cum abdomine capite cum pronoto vix duplo longiora; oculi pronoto quam maxime approximati; pronoti dimidium anticum sensim coarctatum; antennae vix longitudine insecti; quarum articulus primus crassior secundo, brevior tertio, secundus tenuior apice tantum crassior, plus quam duplo longior primo, quartus tenuissimus; hemielytra binervia; membrana binervis, cellulis duabus; femora intermedia anticis longiora et crassiora, postica longiora et crassiora intermediis; libiae femoribus valde longiores. u. Pronoti margine postico subrecto. Omnes planiusculi. *. Antennarum articulo secundo apice modice incrassato; variegatus.

Spec. 84. Fulvomaculatus. De Ger. Ins. III. 294. 33.

" Fall. I. C. S. 46.
") II. S. 88.24.
Zetterst. F. L. I. 489.9.


Fulsomaculatus. IIerrich - Schaeff. III. p. 20. t. $8 \bar{i}$. f. $26 \bar{\tau}$. ิิ. III. p. 81. t. 99. f. 302.

") Herrich-Schaeff. N. E. I. 48 et 84 .

Zetterst. J. L. 273. 16. 10. Neyer. I. 96. 84.

Rufo-brunneus, aureo-pilosus, pirmoto obscure fuscomaculato, hemielytris fusco-pallidis aut pallide-coccincis, appendice luteo apice nigro, membrana fusca, pedibus ferrugineis aut testareis, antennis rufis, articulo quolibet apice fusco.

Long: 3 lin.

Lat: 1 lin.

Habitat in Salice Caucasi.

* Antennarum articulo serundo apice ralde incrassato; fasciatus.

Spec. 85. Sphegiformis. Rossi. F. E.

Clesatus. Liun. S. N. II. 729. 97. (teste Fallén.) Bifusciatus. Fabr. E. S. IV. 177. 152.

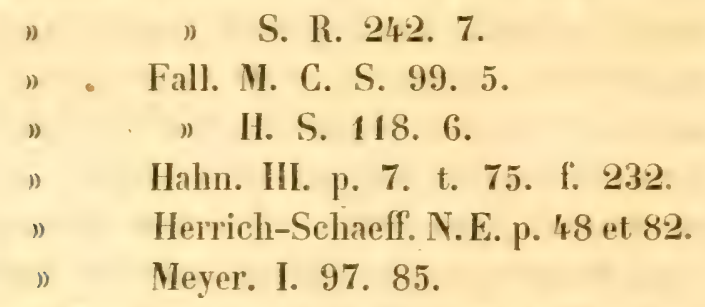

Piceus, auren-pilosus; antennis pedibusque rufis, antennarum articulo primo aut rufo aut nigro, secundo ab apice usque ad dimidium incrassato, nigro; pronoti margine postico, hemielytrorum externo et puncto laterali luteis; alpendice piceo, medio fascia lutea; membrana fusca. 


\section{$-111-$}

Long: 3 lin.

Lat: 1 lin.

Ilabitat in Corylo Caucasi.

+ Unicolor; nigro-fuscus.

Spec. 86. Arbustorum. Panz. F. G. 20.

Fabr. E. S. IV. 175. 141.

" S. R. 238. 174.

Fall. 11. C. S. 68.

1) II. S. 104, 52.

Zetterst. F. L. I. 493.

Herrich-Schaeffer. III. p. 80. t.

99. f. 300 .

))

N. E. I. 53

et 82 .

" Zetterst. J. L. 275. 16. 25.

1) Veyer. 1. 6\%. 33. t. 3. f. 1.

Lugubris. Hahn. II. p. 138. t. 72. f. 225.

Pubescens, formae parallelae; hemielytris fuscis, subnitidis; membrana pone appendicem albida, ceterum fusca; antennis basi nigro-fuscis, apice fuseo-testaceis; pedibus testaceis, subtilissime nigro-irroratis.

Long: 2 lin.

Lat: $2 / 3$ lin.

Habitat in Pruno Caucasi.

++ Bicolor; viridi-coccineus.

Spec. 87. Ferrugatus*). Fabr. E. S. IV. 173. 132.
)
") S. R. 236. 163.
"
Fall. M. C. S. 79. 42. (Lygaeus.)
)
1) II. S. 86.19.

* Non commutandus cum Lopo ferrugato. Meyer. Miride ferrugata. Fallén et dolabrata Fabricii. 
Ferrugatus. Ilahn. I. p. 204. t. 33. f'. 104. Cimex roseo-maculatus. De Geer. J. IV. Mim. 293. 32.

1 Geoff. J. I. 457. 44.

Lygaeus cumpestris. Fabr. E. S. IV. 171. 125. $"$ S. R. 234. 15\%.

(absque citatis!)

Viridis; claro corioque in disco postico roseo aut coccineo; antennis pedibusque ferrugineis apice profundius tinclis; scutello in medio disci lineola nigra; appendix roseus.

$$
\begin{aligned}
& \text { Long: } 3 \frac{1}{2} \text { lin. } \\
& \text { Latit: } 11 / 4 \text { lin. }
\end{aligned}
$$

Ilabitat in Cerealibus Caucasi et Transcaucasiae.

3. Pronoti margine postico rotundato-arcuato. Omnes subconvexi.

[*] Hemielytra cum membrana triplo longiora quam cceput cum pronoto.

+ Appendix flasus.

† Pronotum postice transverse nigrum, nitidum. Spec. 88. Fraxini. Fabr. E. S. IV. 172. 131.

$$
\begin{aligned}
& " \quad \text { S. R. 236. } 162 . \\
& " \quad \text { Hahn. III. p. 82. t. 99. f. } 303 . \\
& " \quad \text { Herrich-Schaeff. N. E. I. } 51 \text { et } 8 \text { 4. }
\end{aligned}
$$

Testaceo-rufescens; antennis, capite pedibusque purpureis: appendicis basi coriique apice purpureis, cuneo apice-nigro; pronoto antice et in margine postico luteo, fascia transversa nigra nitida; scutcllo rufo.

$$
\begin{aligned}
& \text { Long: } 3^{1} / 2 \text { lin. } \\
& \text { Lat: } 1^{1 / 3} \text { lin. }
\end{aligned}
$$

Habitat in Umbellatis provinciae Karabagh et Elisabrethopoleos, Transcaucasiae. 
$\dagger \dagger$ Pronotum nitidum, medio maculis quatuor, postice fascia transversa, nigris.

Spec. 89. Striatellus. Panzer. F. G. 93. 17.

" Fabr. E. S. IV. 173. 133.

" S. R. 236.164.

"Wolff. Jc. C. 153. 150. t. 15.

f. 150. a. b.

» Fallén. M. C. S. 78. 38.

$"$ " H. S. 84. 15.

") Zetterst. F. L. I. 488. 7.

"Hahn. II. p. 133. t. 71. f. 218.

D Herrich-Schaeff. N. E. I. 51 et 87.

" Zetterst. J. L. 272. 16. 8.

" Meyer. I. 94. 81.

Viridi-flavus; hemielylris pone nervos nigro-aut fusco-striatis; appendicis apice nigro; antennis pedibusque testaceis apice fuscis aut rufis; femora annulis duobus obsoletis purpureis.

Long: $3^{1} / 2$ lin.

Lat: $1 \frac{1}{4}$ lin.

Ilabitat in Umbellatis Armeniae et Caucasi.

$\dagger \dagger \dagger$ Pronotum opacum, medio maculis duabus nigris. Spec. 90. Chenopodii*). Fall. M. C. S. 711. 25.

$"$ H. S. 77. 1.
$" \quad$ Herrich-Schaeff. N. E. I. 83.
Meyer. I. 51. 11.

Miris laevigatus. Panz. F. J. G. 23. 21.

n) Wolff. Je. Cim. 36.

Lygaeus it punctatus. Fabr. E. S. IV. 172. 128.

*) Denominatio etsi serius edita accipienda ob Synonima varia et ad praecavendos errores. 
Lygaeus 4 punctatus. Fabr. S. R. 235. 157. (Teste Fallén).

)) Donovan. III. pl: 101. 1. 2.3 .

Bipunctatus. Fabr. E. S. IV. 173. 129.

S. R. 150 .

Binotatus. Fallén. H. S. 78. 3.

" Hahn. 1. p. 202. t. 33. f. 103.

1) Herrich-Schaeff. N: E. I. 50 et 82.

Viridi-pallescens, pilosa, pubescens; pronoto ad latera puncto nonnumquam altero nigro; antennis ferrugineis apice nonnumquam fuscis; hemielytris macula media triangulari, oblonga, nigra aut fusca; pedilus testaceis, rufo-adspersis.

$$
\begin{aligned}
& \text { Long: } 4-41 / 5 \text { lin. } \\
& \text { Lat: } 1 \frac{1}{3} \text { lin. }
\end{aligned}
$$

Habitat in herbis vialibus et vervactorum, praeprimis Verbasco, Cruciferis et Lmbellatis, Caucasi et Transcaucasiae.

++ Appendix aurantiacus.

- Scutellum nigrum aut nigro-fuscum. Spec. 91. Seticornis. Fabr. E. S. IV: 179. 160.
D S. R. 244.18.
»Wolff. Jc. C. 111.
„ Burm. Handb. II. 269.

Tibialis. Wolff. Jc. C. 111.

Lateralis. Fall. H. S. 88. 23.

Hahn. I. t. 35. f. 114. A. a.

Herrich-Schaeff. N. E. I. 51 et 85.

Neyer. I. 95. 83.

Apicalis. Hahn. I. p. 220.

Fulso-maculatus. Fall. I. C. S. 46. var. $\gamma$. 


\section{$-115-$}

Niger, aureo-pilosus; pronoli nigro-fusci margine postico lineolaque longitudinali tenuissima obsolete luteis; hemielytris rufo-fuscis; appendicis apice nigro; membrana fusca, pedibus fuscis, nigris aut rufis.

\section{Long: 4 lin.}

Lat: $1 \frac{1}{2}$ lin.

Habitat in Urticis Caucasi et lberiae.

$\odot$ ๑ S Scutellum nigrum apice luteo. Spec. 92. Scriptus*). Fabr. S. R. 153.

" $\cdots$ Herrich-Schaeff. III. p. 86. t. 97.

f. $29 \%$.
(absque citatis.)

Citrinus, viridi-luteus, nigroque pictus, subtus niger, marginibus segmentorum luteis; capite et pronoto luteo, vittis in priori duabus, in altero quatuor longitudinalibus nigris, vittis in pronoto antice confluentibus; hemielytra luteo - aurantiaca, vittis tribus longitudinalibus nigris in embolyo confluentibus, vitta externa cum media in area corii jam confluenti apice sub angulo fere recto extrorsum flexa et in fasciam transversam dilatata, vitta interna in margine scutellari et commissura clavi; appendicis apex niger; membrana fusca; antennae aut integrae rufae aut articulo tantum primo rufo, apice fusco-nigrae; femoribus basi nigris, ceterum rufis; tibiis rufis, nigro - spinosis.

*) Non confundendus cum Lygo scripto. Fabr. E. S. 171 et Phytocoride scripta. Hahn. II. f. 202. marginello. Falor. a quibus differt: Magnitudine, antennis numqguam integris nigris. scutello apice lute. thorace flavo-striato et vitta nigra exterwa apice hemicly-trorum transversa. 


$$
\begin{aligned}
& \text { Long: } 4 \frac{8}{4} \text { lin. } \\
& \text { Lat: } 1 \frac{1}{2} \text { lin. }
\end{aligned}
$$

Habitat in Umbellatis provinciae Elisabethopol, Transcaucasiae.

+++ Appendix viridis.

○ Nitidi.

* Membrana immaculata.

Spec. 93. Affinis. Herrich-Schaeff. N. E. 49 et 82.

" Meyer. I. 48. 6. t. 1. f. 5.

Pabulinus. Fall. M. C. S. 75. 28.

Zetterst. Faun. L. I. 486. 2.

Viridis; membrana viridinervis, antennarum articulo secundo apice fusco; pedibus viridi-testaceis.

Long: 3 lin.

Lat: $1 \frac{1}{8}$ lin.

Habitat in Caucasi Salicibus aut Crataegis.

* Membrana fusco-maculata.

Spec. 94. Contaminatus. Fall. M. C. S. 76. 29.

$" \quad$ H. S. 79. 5.
$" \quad$ Zetterst. F. L. I. 487. 3.
Hahn. I. p. 151. t. 33. f. 76.
Herrich-Schaeffer. N. E. I. 49
et 83.
Zetterst. J. L. 272. 16. 3.
Meyer. I. 45. 1.

Viridis; corio ante appendicem macula fusca; antennis pedibusque flavo - viridibus; femoribus apice bimaculatis tibiisque fusco-spinosis.

$$
\begin{aligned}
& \text { Long: } 2^{1 / 2} \text { lin. } \\
& \text { Lat: } 1 \text { lin. }
\end{aligned}
$$

Habitat in Betula, Crataego et Salice Cancasi.

○ Pubescentes. 
* Membrana fusca.

Spec. 95. Pabulinus. Linn. S. N. II. 727. 83.

" $\quad$ F. S. 947.
$" \quad$ Ge Geer. III. 293. 31.
Geoffr. I. 456. 43.
Scop. E. S. 132. 384. cum var:
1 et 2; exclusa 3.
Schrank. En. 564.
Fabr. E. S. IV. 184. 5.
" $\quad$ S. R. 254. 5.
Schrank. F. b. 1161.
Zetterst. F. L. I. 486. 2.
Hahn. I. p. 149. t. 23. f. 74.
Herrich - Schaeff. N. E. I. p. 49
$\quad$ et 85.
Burmeist. H. II. 270.
Zetterst. J. L. 272. 16. 2.
Meyer. I. 48. 7. t. 1. f. 3.

Viridis; antennis pedibusque viridi - flavis.

Long: $3 \frac{1}{2}$ lin.

Lat: $1 \frac{1}{8}$ lin.

Habitat in Viburno Gaucasi.

** Membrana hyalina.

Spec. 96. Nassatus. Fabr. E. S. IV. 174. 135.

» $)$ S. R. $236,167$.

„ Fall. M. C. S. 76. 31.

" H. S. 80. 7.

1) Zetterst. F. L. I. 487. 4.

" Hahn. I. p. 153. t. 24. f. 78.

1) Herrich-Schaeff. N. E. I. p. 49 et 85.

"Zetterst. J. L. 272. 16. 4. 
Nassutus. Meyer. I. 50. 8.

Icterocephalus. Hahn. I. p. 149. t. 33. t. 75.

Viridis; hemielytrorum margine externo, antennis pedibusque flavis.

Long: $2^{1} / 2$ lin.

Lat: 1 lin.

Habitat in Betula, Crataego et Salice Caucasi.

[**] Hemielytra cum membrana dupplo longiora quam caput cum pronoto.

- Pubescentes. Spec. 97. Campestris. Linn. S. X. II. 728. 87.

" $\quad$ F. S. 950.
$" \quad$ Geoff. I. 452. 34.
$" \quad$ Fall. I. C. S. 83. 51.
Zetterst. F. L. I. 489. 11.
"Hahn. I. p. 218. t. 35. f. 113.
" Herrich-Schaeff. N. E. I. 51 et 83.
Zetterst. J. L. 273. 16. 13.

Supra impresso-punctata, testaceo-virescens aut ferruginea; scutello albo-maculato, hemielytrorum medio macula infuscata.

$$
\begin{aligned}
& \text { Long: } 2-2^{2} / 3 \text { lin. } \\
& \text { Lat: } 1 \text { lin. }
\end{aligned}
$$

Habitat in campis et nemoribus Caucasi.

0 ○ Nitidae.

+ Membrana fusca.

Spec. 98. Punctuta. Zetterst. J. I. 273. 16. 14.

Profunde punctata, subfusca; supra testaceo - ferruginea, fusco-maculata; pronoti parte antica tuberculosa et angulis posticis nigris.

Long: $2 \frac{1}{2}$ lin.

Lat: 1 lin. 


\section{$-119-$}

Habitat in pratis subalpinis Caucasi.

++ Membrana maculis fuscis.

$\dagger$ Pronoti angulis posticis flavis.

Spec. 99. Pratensis. Linn. S. N. 728. 86.

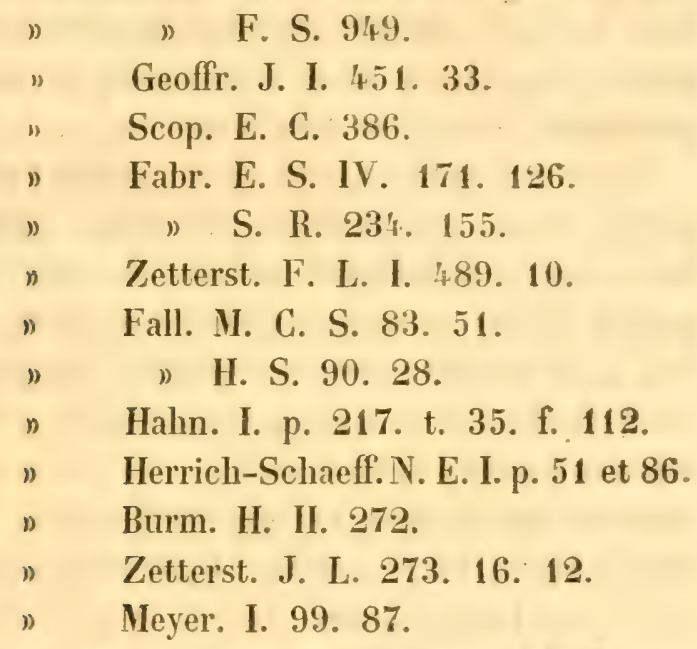

Supra impresso - punctata, sparse pilosa; viridi flavescens aut ferruginea; scutello albo- aut luteo-cordato; femoribus annulis duobus ferrugineo-fuscis; antennarum articulo primo et secundo rufo, secundo apice reliquisque fusco-nigris.

$$
\begin{aligned}
& \text { Long: } 2^{2} / 3-2^{4} / 5 \text { lin. } \\
& \text { Lat: } 1-1^{1 / 3} \text { lin. }
\end{aligned}
$$

var: Umbellatarum. Panz. F. G. 93. 19.

Rufipennis. " Fall. M. C. S. 84. 22.

„) Herrich-Schaeff. N. E. I. 83. var. 2.

Viridi-rufa; hemielytris nervoque membranae sanguineis.

Habitat in pratis Caucasi et Transcaucasiae, ad altitudinem $\mathbf{7 0 0}$ orgyiarum gallicarum.

$\dagger \dagger$ Pronoti angulis posticis nigris. 
Spec. 100. Alpina. m.

Vide: Tab. X. fig. 24.

Viridi-flava aut ferruginea, crebre punctata, nitidissima; pronotum striis quatuor longitudinalibus aut maculis octo aut quatuor vel duabus, angulis posticis semper nigris; hemielytra pone appendicem maculis duabus nigro-piceis; ventre macula media nigra.

Caput ferrugineum aut flavum; antennarum articulus primus rufus, secundus aut in medio rufus apicibusque fuscis aut cum articulis reliquis fuscus: femora subtus macula picea, apice annulis duobus piceis aut rufis; $t i$ biis apice piceis; scutellum pallide-luteum; hemielytra viridi-flava aut ferruginea; apice corii ad marginem externum maculis duabus piceis, ad commissuram macula coccinea aut purpurea; appendice pallido in angulis piceo; cellulue nervo pallido; tegminibus opalizantibus.

Long: 3 lin.

Lat: $1 \frac{1}{5}$ lin.

Habitat in Armeniae alpilus, monte Ssarijal, Kaepesdagh, Murgus et Alaghoes.

- Appendice-luteo, apice nigro; scutello luteo-cordato.

$x$ Capite ferrugineo, reliquis magna ex parte nigris. Spec. 101. Tripustulatus. Fabr. E. S. IV. 176. 145.

\begin{tabular}{|c|c|}
\hline & S. R. 239.182. \\
\hline & Fall. H. S. 96.38. \\
\hline & Hahn. I. p. 215 . t. 34. f. 111. \\
\hline & $\begin{array}{l}\text { Herrich-Schaeff. N. E. I. } 52 \\
\text { et } 88 \text {. }\end{array}$ \\
\hline & Burm. II. 273. \\
\hline & Zetterst. J. L. 275. 16. 21. \\
\hline & Meyer. I. 106. 96. \\
\hline
\end{tabular}

Transcersalis. Fabr. E. S. IV. 175. 142. 
Transversalis. Fabr. S. R. 238. 175.

") Herrich-Schaeff. N. E. I. 87.

Bistriguttutus. Fabr. E. S. IV. 174. 135.

$" \quad$ S. R. 236. 166.

Pastinacae. Fall. M. C. S. 86. 57.

$"$ H. S. 94. 35.

" Hahn. I. p. 213. t. 34. f. 110.

Pictus. Hahn. I. p. 215.

Niger, nitidus; antennis, pedibus, capite et macula antica pronoti rufis; femoribus apice annulis tribus, tibiis annulo in medio et apice piceis; appendice nigro. macula media aurantiaca magna; membrana fusca.

Caput rufum, apice nonnumquam piceum; antennarum articuli apice nonnumquam infuscati; pronotum nigrum, antice rufum, linea media longitudinali et marginibus subtilissimis rufis; scutellum basi nigrum, ceterum cordato viridi - flavum aut ferrugineum; hemielytra nigra, macula embolyi et fascia media corii ferruginea; abdomen nigrum, apice et marginibus rufis.

Long: 2 lin.

Habitat in Caucasi Umbellatis et Urticis. Var: 102. Lhesgica. m.

(Verosimiliter pluribus exemplaribus conspectis novam referet speciem!)

Pronoto nigro, margine postico subtilissime luteo; antennarum articulo primo rufo, reliquis nigro-piceis; hemielyira macula una lata lutea ab embolyo arcuata-triangulari ad medium corii, altera minori in commissura clavi; appendice nigro, macula media Intea magna, apice latius nigro; femoribus annulis duobus, tibiis totis piceis; abdomine viridi-luteo, marginibus apiceque nigro. 
Long: $1 \frac{4}{5}$ lin.

Lat: $4 / 5$ lin.

Habitat in pratis subalpinis montis Ssalwat, Caucasi Lhesgici.

$\times \times$ Capite luteo, reliquis magna ex parte luteis. Spec. 103. Kalmii. Linn. Fn. S. 948.

Zett: F. L. I. 491. 15.

Herrich-Schaeffer. N. E. I. 84 .

Zetterst. J. L. 274. 16. 20.

Meyer. I. 105. 95.

Gramineus. Fabr. E. S. Suppl. 542. 154.

" $\quad$ S. R. 243.11.

Flavovarius. Fabr. E. S. IV. 178. 154.

" $\quad$ S. R. 243.10.
$" \quad$ Fall. M. C. S. 86.53.
" H. S. 93.34.
Hahn. I. p. 211. t. 34. f. 109.
Burm. II. 172.

Viridi-flavus; thorace antice et postice nigro; scutello luteo; hemielytris rufo-flavis apice fascia nigra; appendice luteo, apice nigro; membrana fusca; antennis pedibusque testaceis; abdomine luteo, fasciis tribus longitudinalibus nigris.

Long: $1 \frac{4}{3}$ lin.

Lat: $4 / 5$ lin.

Habitat in dumetis Caucasi, in Humulo et Parietaria praeprimis.

$\odot \odot$ Appendice medio ocellato, scutello nigro, apice luteo-rufo.

$x$ Hemielytris nigris.

Spec. 104. Gyllenhalii. Fall. M. C. S. 88. 61.

»... H. S. 97. 40 . 
Gyllenhalii. Herrich-Schaeff. III. p. 86. ᄂ. 101. t. 310 .

$\Rightarrow \quad$ N. E. I. p. 52
et 84 .

n) Meyer. I. 61. 28.

Niger, opacus, luteo-pubescens; appendice luteo, medio macula magna orbiculari nigra; apice summo scutelli luteo aut rufo; membrana declivi; pedibus rufis, femoribus nigro-annulatis aut nigris; antennis rufis aut fuscis.

\section{Long: $1^{2} / 3$ lin. \\ Lat: ${ }^{4} / 5$ lin.}

Habitat in Rubiaceis, praeprimis Galiis Caucasi.

$\times \times$ Hemielytris nigris, luten-variegatis.

Spec. 105. Unifasciatus. Fabr. E. S. IV. 178. 153.

$\begin{array}{ll}" \quad & \text { S. R. 243. } 9 . \\ " & \text { Zett. F. L. I. 491. 15. } \\ \text { Herrich - Schaeff. N. E. I. } 51 \\ \text { et } 88 . \\ \quad \text { Zetterst. J. L. 274. 16. } 20 . \\ \text { Meyer. I. 104. } 93 .\end{array}$

Semiflasus. Wolff. IV. 154. t. 15. f. 48.

D Fall: M. C. 80, 44.

》) H. S. 86, 21 .

" Hahn. I. p. 208. t. 34. f. 107.

$\left.\begin{array}{l}\text { Lateralis. } \\ \text { Marginatus. }\end{array}\right\} \begin{aligned} & \text { Uahn. II. p. } 85 . \text { t. 56. f. } 169 . \\ & \text { 170. }\end{aligned}$

Niger-pubescens; pronoti margine postico, scutelli dimidio apicali et hemielytris ad embolyum et dimidium corii angulumque clavi luteis; appendice aurantiaco aut luteo, macula media nigra; membranae fuscae nervo luteo; antennae pedesque rufo-testacei. 
Long: 2 lin.

Lat: 1 lin.

Habitat in Galiis Caucasi.

$\odot \odot \odot$ Appendice roseo.

Spec. 106. Rubricatus. Fall. M. C. S. 91. 70.

II. S. 100. 45.

Zett. F. L. I. 493. 21.

) J. L. 275. 16.

"Hahn. I. p. 156. t. 24. f. 80. (Lygus.)

" Herrich - Schaeffer. N. E. I. 49 et 86 .

$1 \quad$ Meyer. I. 73.45.

Lygus rufescens. Hahn. I. p. 28. t. 4. f. 18.

Flavo-ruber; pronoto antico capiteque nonnumquam pallidioribus; femoribus annulis duobus ferrugineo-fuscis.

Long: $1^{3} / 4-2 \frac{1}{2}$ lin.

Lat: $3 / 4$ lin.

Habitat in Salice Caucasi.

$\odot \odot \odot \odot$ Appendice cum tota superficie concolori.

$\times$ Viridis, nitidus.

Spec. 107. Viridulus. Fall. M. C. S. 90. 69.

1 H. S. 105. 54.

1) Hahn. II. p. 136. t. 72. f. 221.

1 Herrich-Schaeff. N. E. I. 50 et 88.

D Meyer. I. 77. 51. t. 7. f. 2.

Nigro-sparse pilosus; pedibus nigro-punctatis; antennarum articulus primus medio, secundus basi fuscus.

Long: $14 / 5$ lin.

Lat: $2 / 3$ lin.

Habitat in Betulis pratisque alpinis Caucasi.

$\times \times$ Viridis, opacus. 
Spec. 108. Hortulanus. Meyer. I. 77. 52. t. 7. fig. 3.

Pallide-virescens; albo-pilosus, antennis pedibusque pallidis.

Long: $1 \frac{1}{2}$ lin.
Lat: $1 / 2$ lin.

Habitat in pratis Armeniae et Iberiae.

$\times \times \times$ Albidus, opacus.

Spec. 109. Albida. m.

Vide: Tab. XI. fig. 27.

Supra albidus alboque pilosus; pedilus pallide-testaceis, antennis abdomineque nigris.

Long: $\mathbf{1}^{2} / 3$ lin.

Lat: $3 / 5$ lin.

Ilabitat in pratis provinciae Karabagh, Transcaucasiae.

c. Pronotum subconsexum, impressione in medio antico levi, antennarum articulo secundo ad dimidium aut plus incrassato.

Genus: HETEROTOMA. Latr.

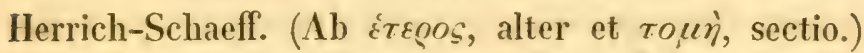

Capsus. Fab. R. Fall. Latr. Hahn. Herrich - Schaeff. Meyér. Phytocoris. Hahn. Poecilosoma. Steph. Lygaeus. Fabr. E. Wolff. Panz. Halticus. Burm. Ci$m e x$. Linné. Geoffr. Pallas.

Character: Forma ovalis; hemielytra convexa, nervis duobus; membrana cellula una; antennarum articulo primo capitis longitudine, secundo omnium longissimo plus quam dimidio incrassato, tertio quartoque subtilissimo molli ac setiformi, quarto in medio paulo impresso aut incurvato; rostellum ad acetabula pedum posticoram protractum, cujus articulus primus longitudine capitis, secundus omnium longissimus, tertius et quartus brevissimus; oculi valde prominuli, pedes crassi. 
a. Forma oblonga, subdepressa.

*. Nigri.

+ Hemielytris pronoto non latioribus.

Spec. 110. Oblongus. m.

Vide: Tab. XI. fig. 28.

Ater, oblongus, linearis, luteo-pilosus, pronoti angulis posticis acutis, rufis; antennarum articulo secundo clavato, hispido; pedibus fuscis.

Long: $2^{1 / 5}$ lin.

Lat: thor: postici $3 / 5$, elytrorum $1 / 2$ lin.

Habitat in Pyro elacagnifolia et Cotoneaster Nummularia provinciae Karabagh prope pagum Tschaikaent.

++ Hemielytris pronoto latioribus.

Spec. 111. Pulrerulentes. Kluge. Burm. H. II. 276.

Meyer. I. 63. 31.

Ater, oblongo-ovalis, albido-pilosus, antennarum articulo secundo clavato, tibiis fuscis aut nigris.

Long: $1^{2} / 3-2$ lin.

Lat: thor: postici: $3 / 5$, hemielytrorum $4 / 3-1$ lin.

Habitat in Caucasi Mespilo et Cydoniis.

** Fusco-rufi.

Spec. 112. Planicornis. Pallas. Spicileg. zool. Fasc. IX. p. 23. tab. I. f. 13. (Cimex.)

Magnicornis. Schellenbg. G. d. W. T. IV. f. $2, \mathrm{C}$.

" $\quad$ Fall. M. C. S. 99. 7.
$" \quad$ I. S. 119. 8.
Zett. F. L. I. 498. 4.
Hahn. I. p. 130. t. 20. f. 67.
" $\quad$ Herrich-Schaeff. N. E. I. p. 85.
Zett. J. I. 278. 17. 4.
Meyer. I. 62. t. II. f. 4.


Niger-rufescens aut nigro-violaceus, luteo-pilosus, antennarum articulo secundo fusiformi incrassato nigro, ultimis setaceis, albo-fuscis; tibiae posticae obsolete nigro - annulatae, spinis longis.

Long: $1 \frac{1}{2}$ lin.

Lat: $3 / 5$ lin.

Irahitat in Cancaso et in territorio Airum provinciae Karabagh loco sic dicto Schamlogh a pinetis denominato.

$\beta$. Forma ovalis, convexa.

*: Hemielytris concoloribus.

Spec. 113. Ater. Linn. S. N. II. 725. 72.

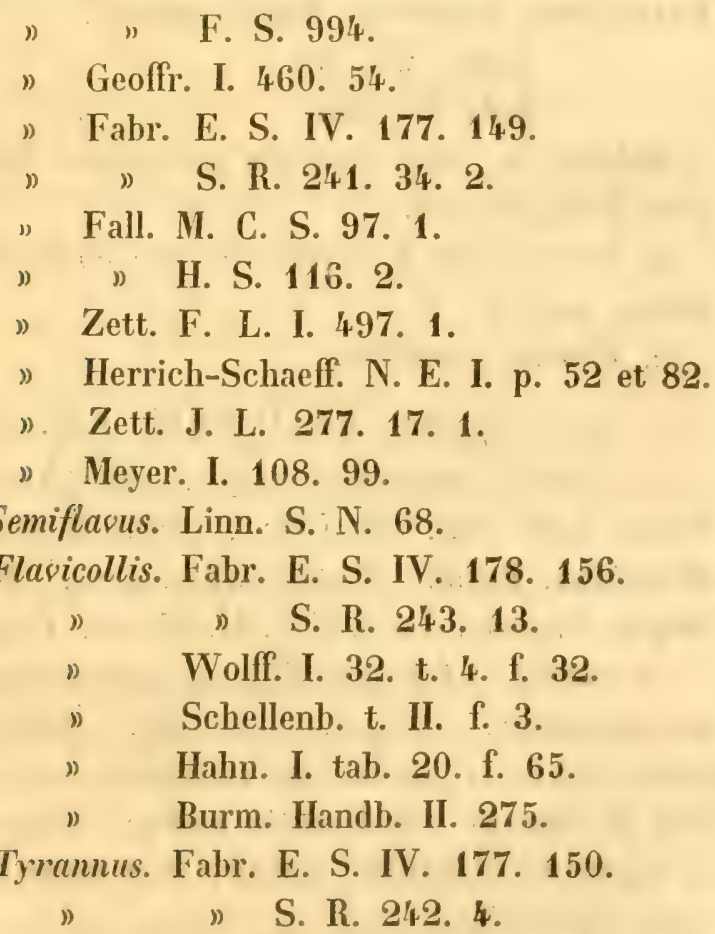

Niger, subnitidus, pronoto capiteque aut nigro aut fer- 


\section{$-128-$}

rugineo, pedibus aut nigris, aut fuscis aut ferrugineis, femoribus apice annulis duobus piceis.

Long: $2^{2} / 3$ lin.

Lat: $1 \frac{1}{s}$ lin.

Habitat in Caucasi et Iberiae pratis herbidis.

* Hemielytris discoloribus.

Spec. 114. Cinctus. m.

Vide: Tab. XI. fig. 29.

Ater, profunde punctatus, subnitidus; capite, thorace, hemielytrorum margine lato externo usque ad apicem appendicis, antennarum articulo primo, coxis pedibusque ferrugineis; femoribus absque annulis.

Long: $2^{1} / 2$ lin.

Lat: $1 \frac{2}{s}$ lin.

Habitat in locis herbidis provinciae trans Caucasum sitae Elisabethopol.

d. Pronotum hemielytraque consexa, impressione nulla.

a. Femora simplicia.

Genus: BRYOCORIS. Fall. H.

(A 3ozion, muscus et róots.) Orthonotus. Westmood. Curt. Cat. (ab jo Halticus. Burm. Anthocoris. Zett. Poecilosoma. Steph. Capsus. Fabr. Fall. I. Neyer. Lygaeus. Wolff.

Character: Antennae filiformes, apice capillares. Corpus minutum, oratum, convexum. Hemielytra coleoptrata, convexa, membranae rudimento aut nullo. Habitant in muscis. Differentia sexus: Femina rima brevi in segmento abdominis apicali, mas segmento eodem integro dignoscitur.

+ Scutello nigro. 
Spec. 115. Filicis. Linn. S. N. 212. 720.

) F. S. 919.

Wolff. II. 46. 43. f. 43.

Fall. II. C. S. 92. 74.

») H. S. 108. 61.

Hahn. II. p. 86. t. 56. f. 172.

n Herrich-Schaeff. N. E. I. 51 et 84.

$₫ \quad$ Meyer. t. 71. 43.

Nigro-piceus, nitidus; capite, pedilus, hemielytrorum margine pallide-luteis, antennis basi testaceis, apice fuscis; membranula fusca.

\section{Long: 1 lin.}

Lat: $3 / 5$ lin.

Habitat in Aspidiis Caucasi et Transcaucasiae.

++ Scutello fulvo.

Spec. 116. Pteridis. Fall. M. C. S. 105. 20.

") H. S. 152. 1.
$" \quad$ Zett. Jns. L. 266. 11. 1.
Burm. II. 278.
Germ. Fn. 10. 13.
Herrich-Schaeff. N. E. I. 53 et 86.
Curtis. Cat. p. 228.
Meyer. t. 114. 109.

Anthocoris coleoptrata. Zett. Act. Holm. 1819.

$$
\text { p. } 74 .
$$

Glabra, nitida, pallida; capile, antennarum apice, pronoti margine antico, ventreque nigris; hypopygio albido; membrana nulla.

$$
\begin{aligned}
& \text { Long: } 1 \text { lin. } \\
& \text { Lat: } 2 / 5 \text { lin. }
\end{aligned}
$$

Habitat ad radices arborum et in muscis regionis sylvosae Caucasi, ubi Pteris aquilina.

October. 1845. 
ß. Femora incrassata.

Genus: EURYCEPHALA. Laporte.

(Ab cianz: latus et *effuty), caput). Attus. Burm. Hahn. Herrich-Schaeff. als citt(o), prosilio).

Orthanotus. Westr. Curtis. Malticus. Iahn. Burm. (ab ü.т\%ns; salticus.) Cupsus. Hahn. Herrich-Schaeff. Meyer. Phytocoris. Fall. H. Lygaeus. Fall. M. Wolff. Salda. Falor. R. Leanthia. Fabr. F. Cicada. Linn.

Character: Antennarum articulus basulis crassus, secundus omuium longissimus, tertius brevior secundo, quartus longior primo, brevior tertio; rostellum ad acetabula pedum posteriorum porrectum, cujus articulus primus brevis, crassus, secundus longior et aequalis tertio, quartus longitudine primi; hemielytra convexa, absque nervis: membrana, si adest in mare, nerro unico aut cellulam formante aut libero; hemiclvtra in femina desunt: tibiue longae et spinosae; forma oralis aut globosa.

* Totus nigro-aeneus.

Spec. 117. Vitidus. Meyer. I. p. 113. 107. tab. VI. f. 4. Profunde scrobiculatus, nitidus; antennarum articulo basali spinoso; pronoti parte antica torulo utrinque transrerso levi et nitilo: hemielytris postice oblique truncatis absque membrana.

$$
\begin{aligned}
& \text { Long: } 1^{5} / 5-1^{4} / 5 \text { lin. } \\
& \text { Lat: pronoti } 3 / s \text {, abdom: } 1^{2} / 5 \text { lin. }
\end{aligned}
$$

Habitat in arenosis ad mare Caspium et Caucasi Lhesgrici usque ad altiturinem 1200 orgviarum Gallicarum.

* Pedibus testaceis.

+ Capite nigro.

Sprec. 118. Pallicornis. Linn. F. S. 894. (Cicada aptera. ...) 
Pallicornis: Fabr. E. S. IV. 69. 5.

S. R. 115.6 .

Wolff. Jc. C. 122.

Fall. M. C. S. 95.80 .

) H. S. 113. 70.

Hahn. I. p. 114, t. 18, f. 61.

Burm. II. 278.

Meser. I. 110. 103.

1) Herrich-Schaeff. N. E. I. 53 et 86.

Arenarius. ๑๋. Hahn. III. p. 34. t. 84. f. 255.

Viger, nitidus, subtilissime punctatus: untennis basi testaceis, apice fuscis; libiis apiceque fimorum testaceis; femina aptera.

\section{Long: $1-1 \frac{1}{5}$ lin. \\ Lat: $\hat{\theta} \tilde{s} / 4, \quad \& \quad$ lin.}

Habitat in pratis arenosis humidis Iberiae.

++ Capite rufo.

Spec. 119. Rufifions. Fall. 11. C. S. 105. 19.

" Herrich-Schaeff. III. p. 110. t.

$$
\begin{array}{ccc} 
& \multicolumn{2}{c}{\text { 108. f. } 338 .} \\
\text { " } & \multicolumn{1}{c}{\text { N. I. p. } 86 .} \\
\text { " } & \text { Burm. H. II. 278. } \\
\text { Ambulans. Fall. I. C. S. 104. 18. } \\
\text { " } & \text { H. S. 126. 20. } \\
\text { " } & \text { Zetterst. J. L. 279. 12. } \\
\text { " } & \text { " Faun. L. 1. 500. 7. } \\
\text { " } & \text { Herrich - Schaeff. III. p. 109. t. } \\
& \text { 108. f. } 335,336,337 .
\end{array}
$$

Niger, nitidus, subtilissime punctatus; podibns flavis: antennarum articulo primo flavo, secundo basi apiceque nigro; femina aptera. 


\section{$-132-$}

Long: 1 lin.

Lat: $1 / 2$ lin.

Habitat in agris et pratis prope pagum Osseticum Görtscheti montis Kasbek, Caucasi centralis, usque ad altitudinem 990 orgyiarum Gallicarum. 
Tab. I. et II $I^{d t}$ in fasciculo $I$. 




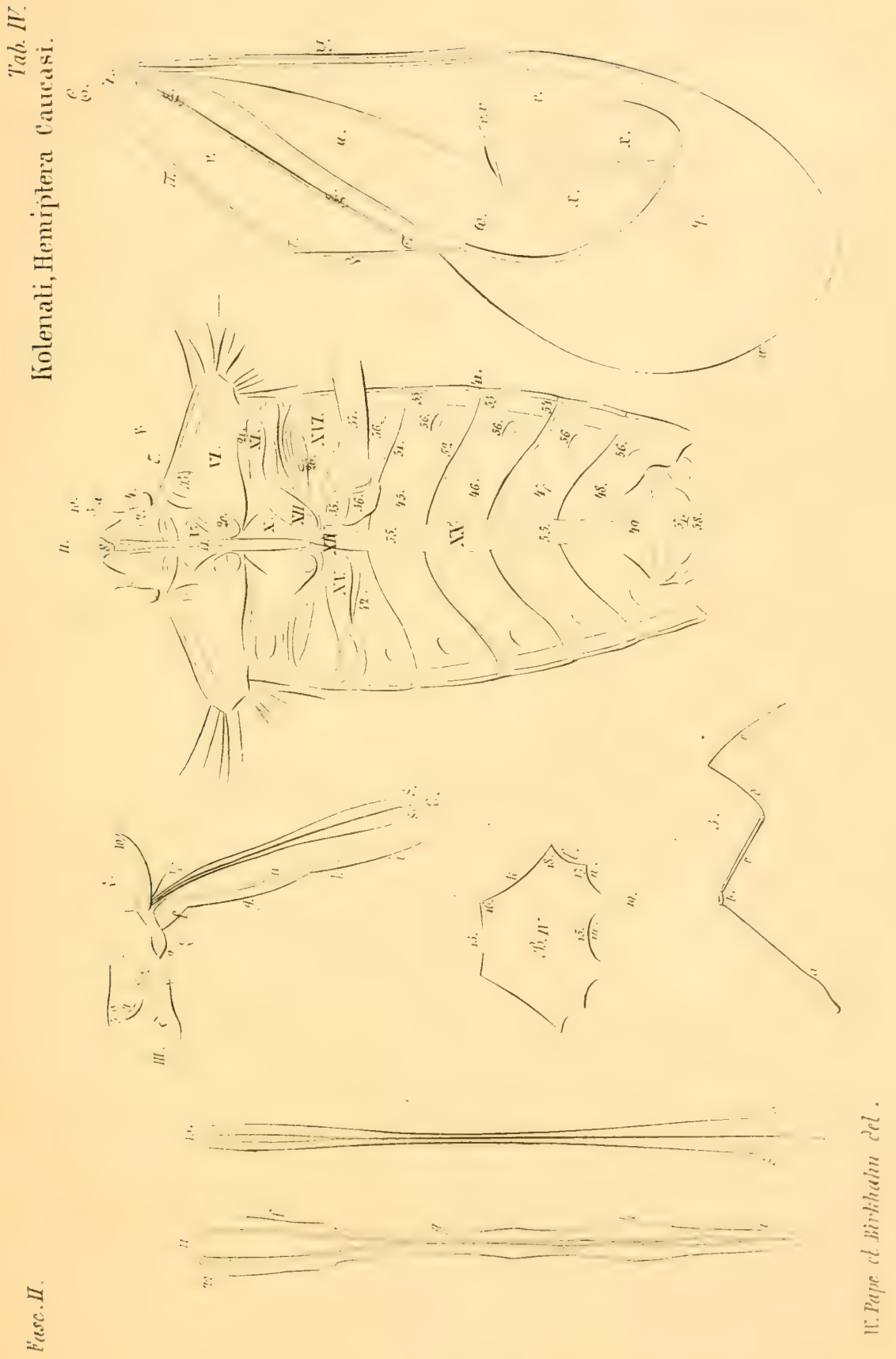







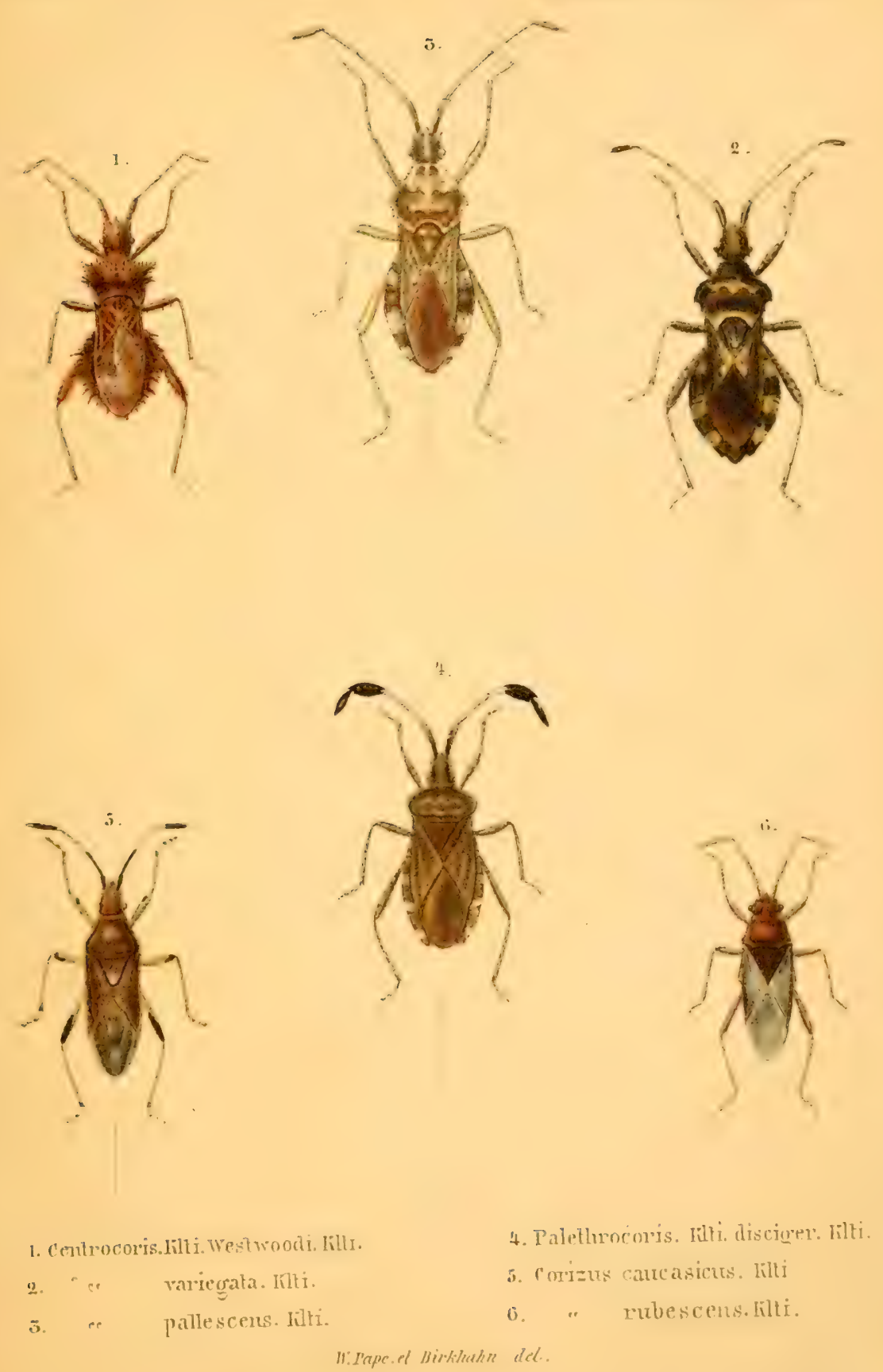




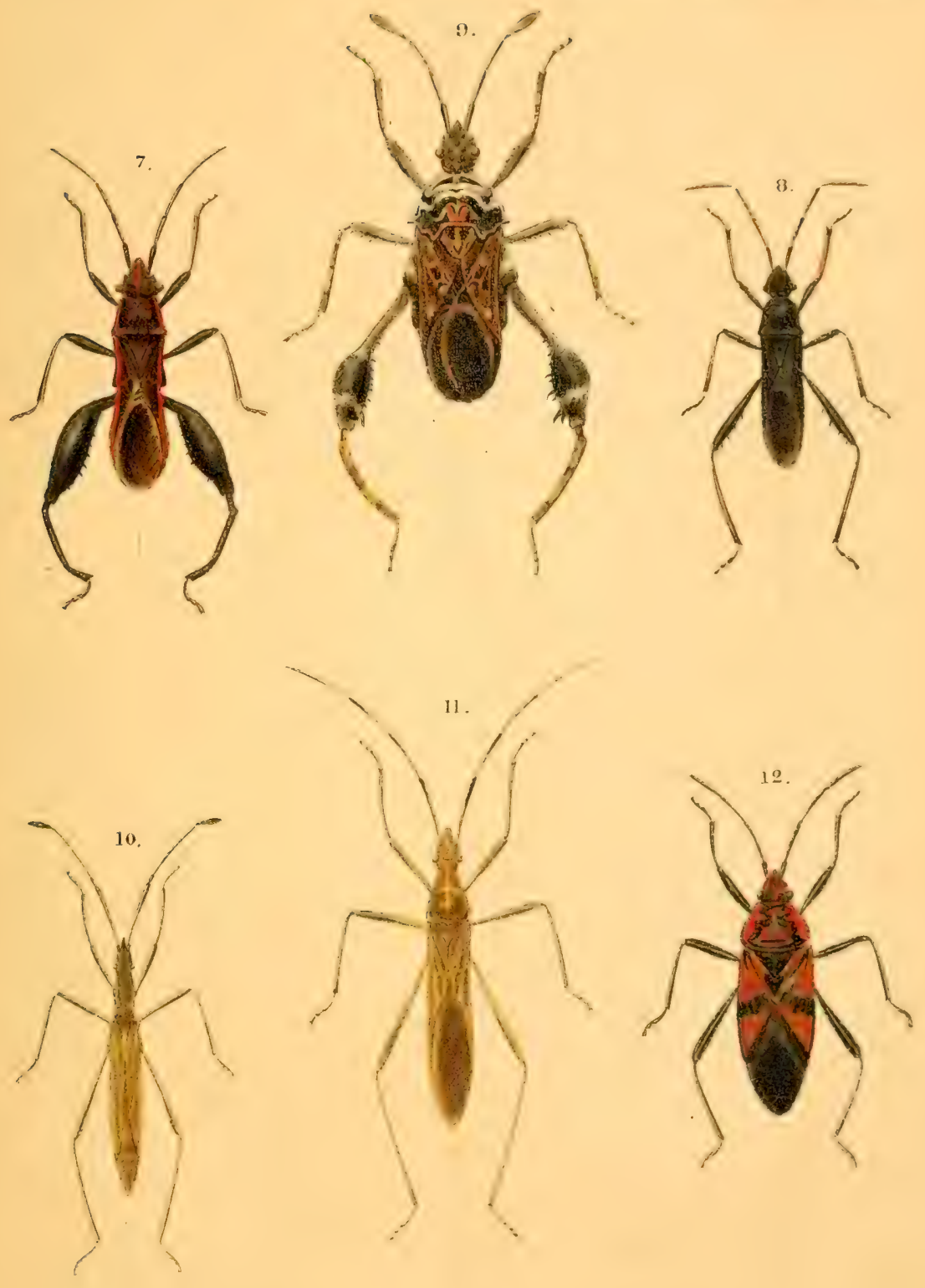

7. Atydus Tragacanthae. Nili.
8. " hirsulus. Iflti.
๑. Hebecerus. Filti. I'ersicus. Killi.

10. Hervlus camasicuth. Filt

11. Rhatrororts kilti. arcuatami.

12. Lyogacus asiaticus. Filti. 


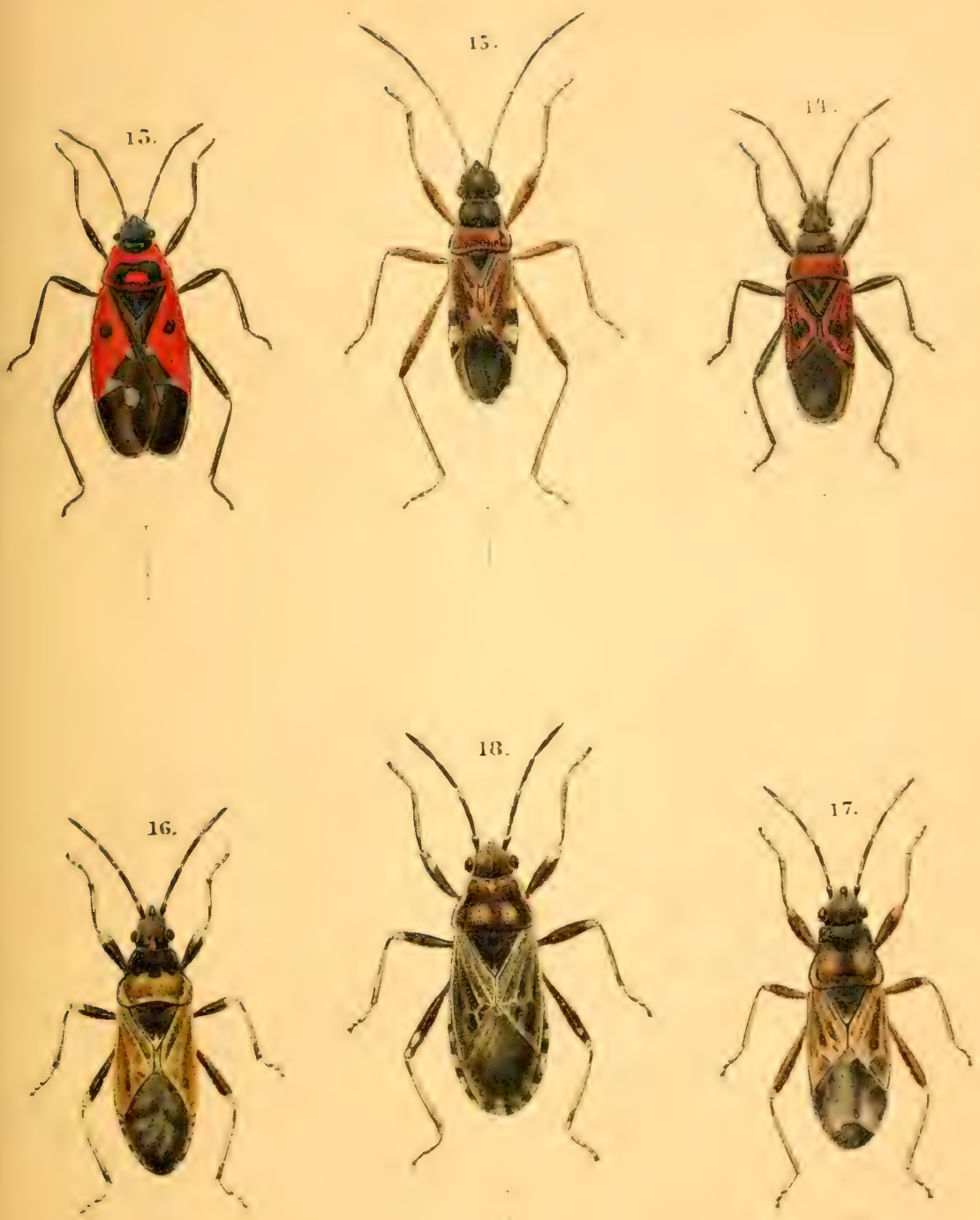

10.. I.ygaeus rentratis. Filti. 14. Pachyme1'us anomalus. Filti.

15. ef Ibericus lilts.
16 Heteronaster bienlor: Tilti. enrmillate.filtr. Walllii.Jilti . 

Tali. I.

Frose. II
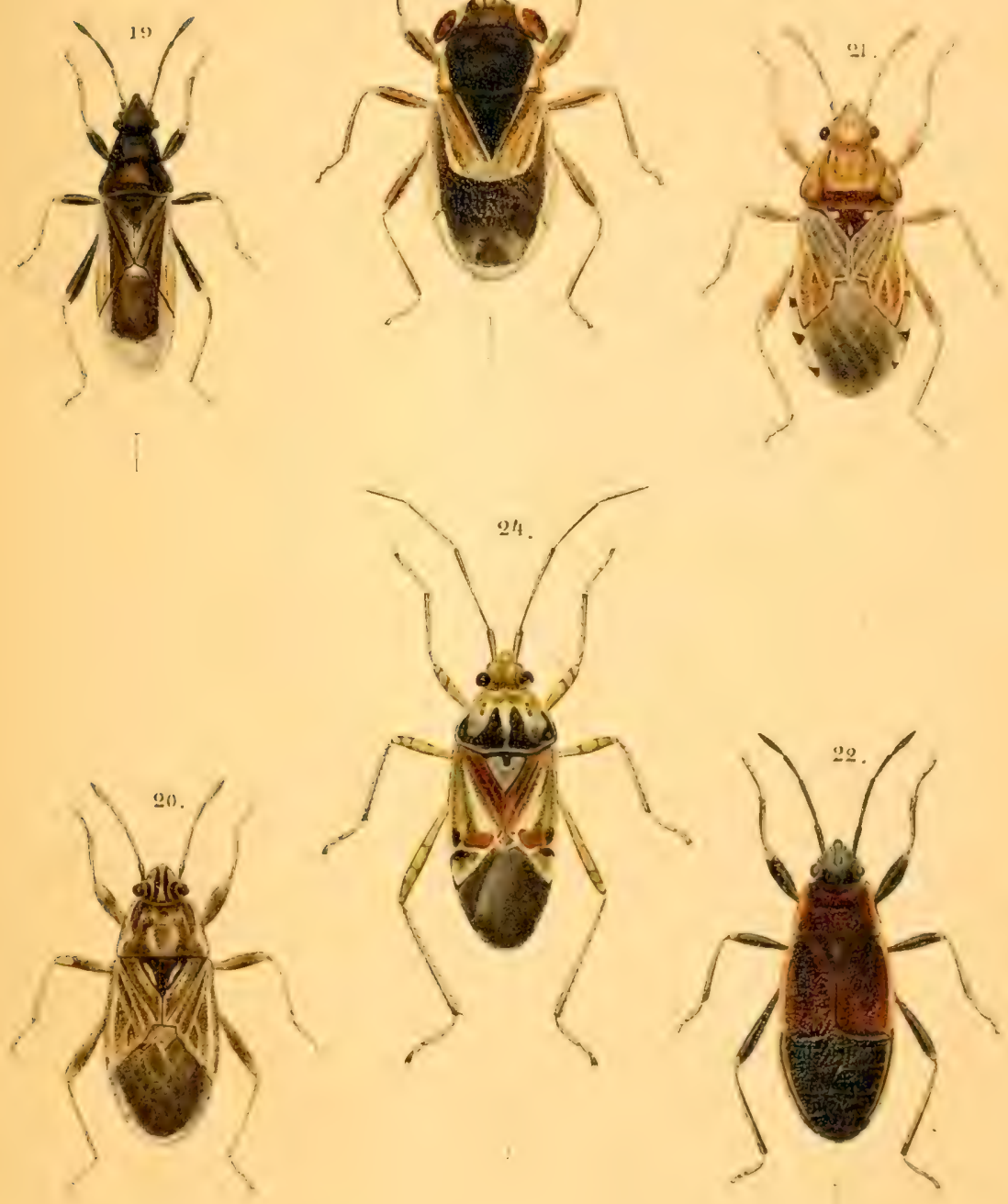

19. ('vmus origani. Jili.

20. of saturejar.lilli.

21. es llp̧reamionstilli.

lolenali, Honipten Cancasi.
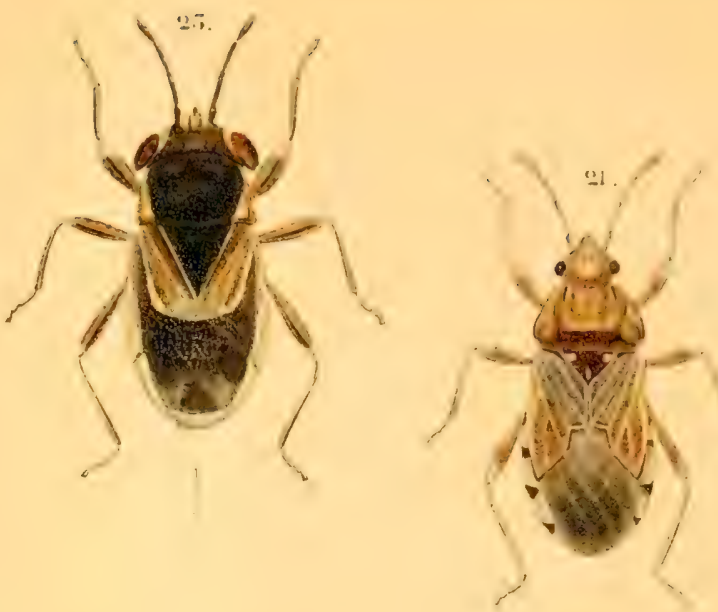

92. Platıogaster manguatus. kilti 25. Cenenis angularis. Fiebres. 2'. Plutucoris alpina lilli 

Tirlo. IT.

Fiesr.ZL.

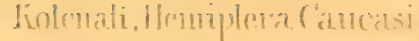

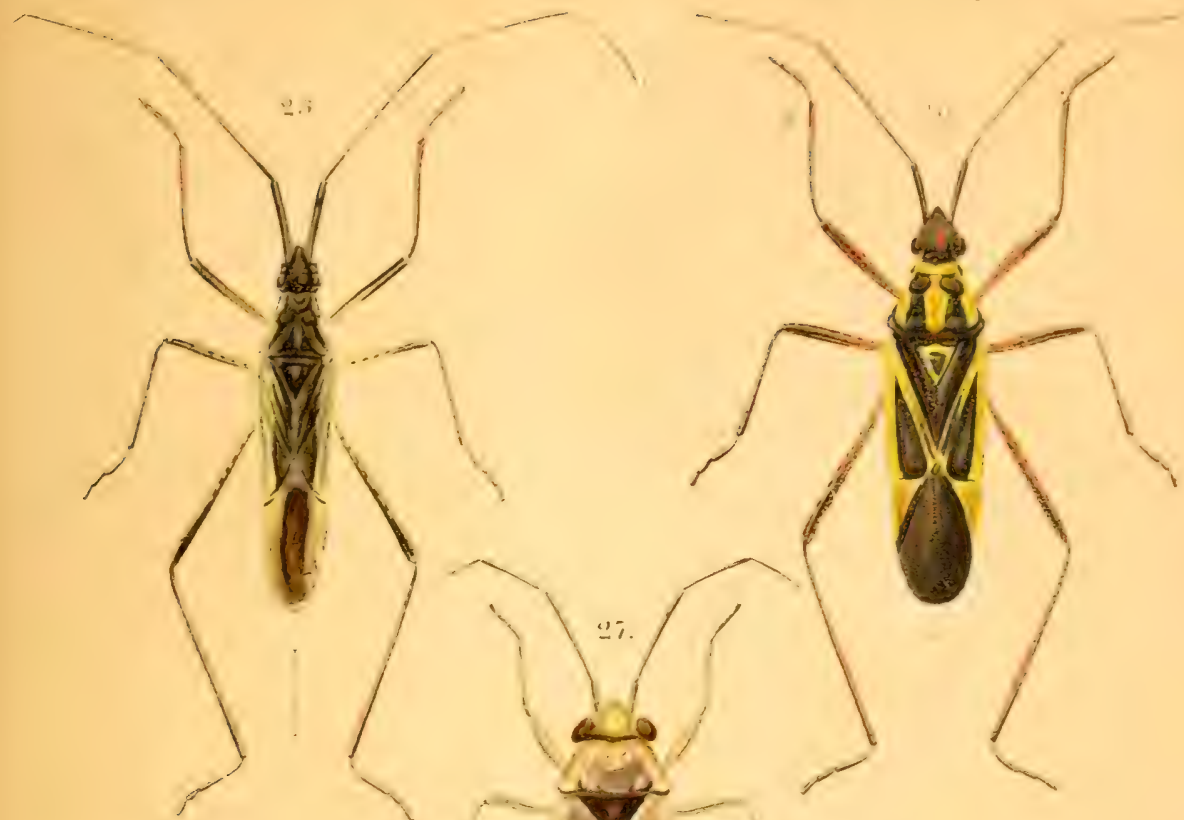

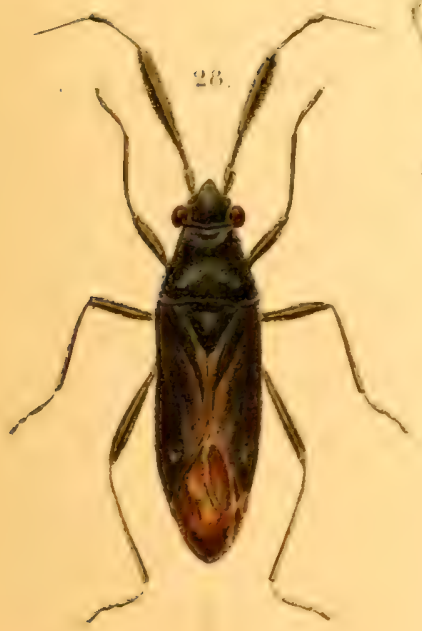

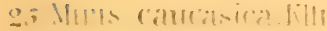

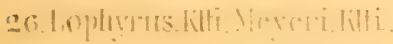

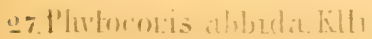

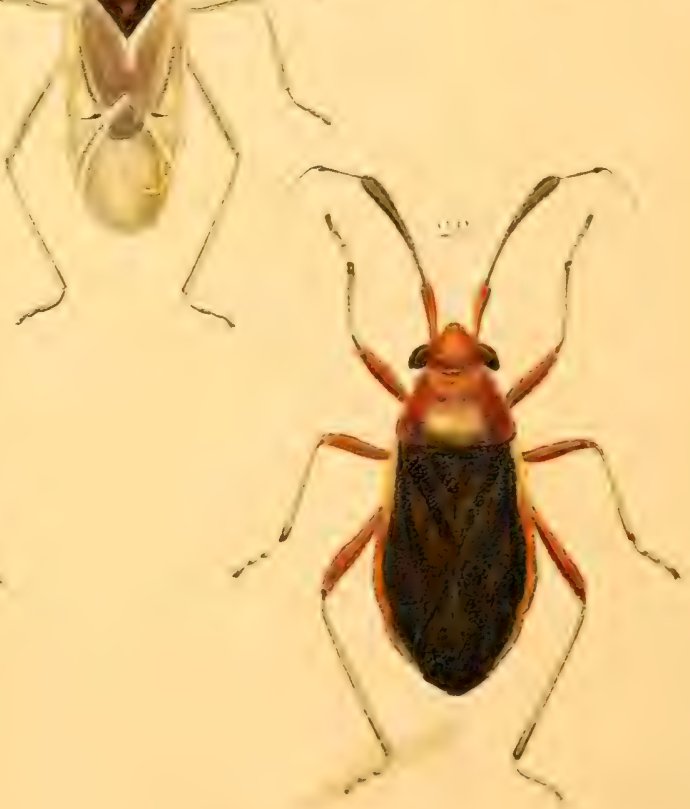

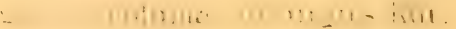

1): 


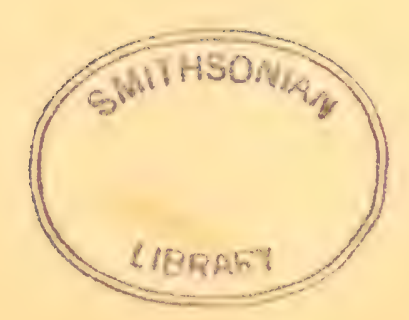





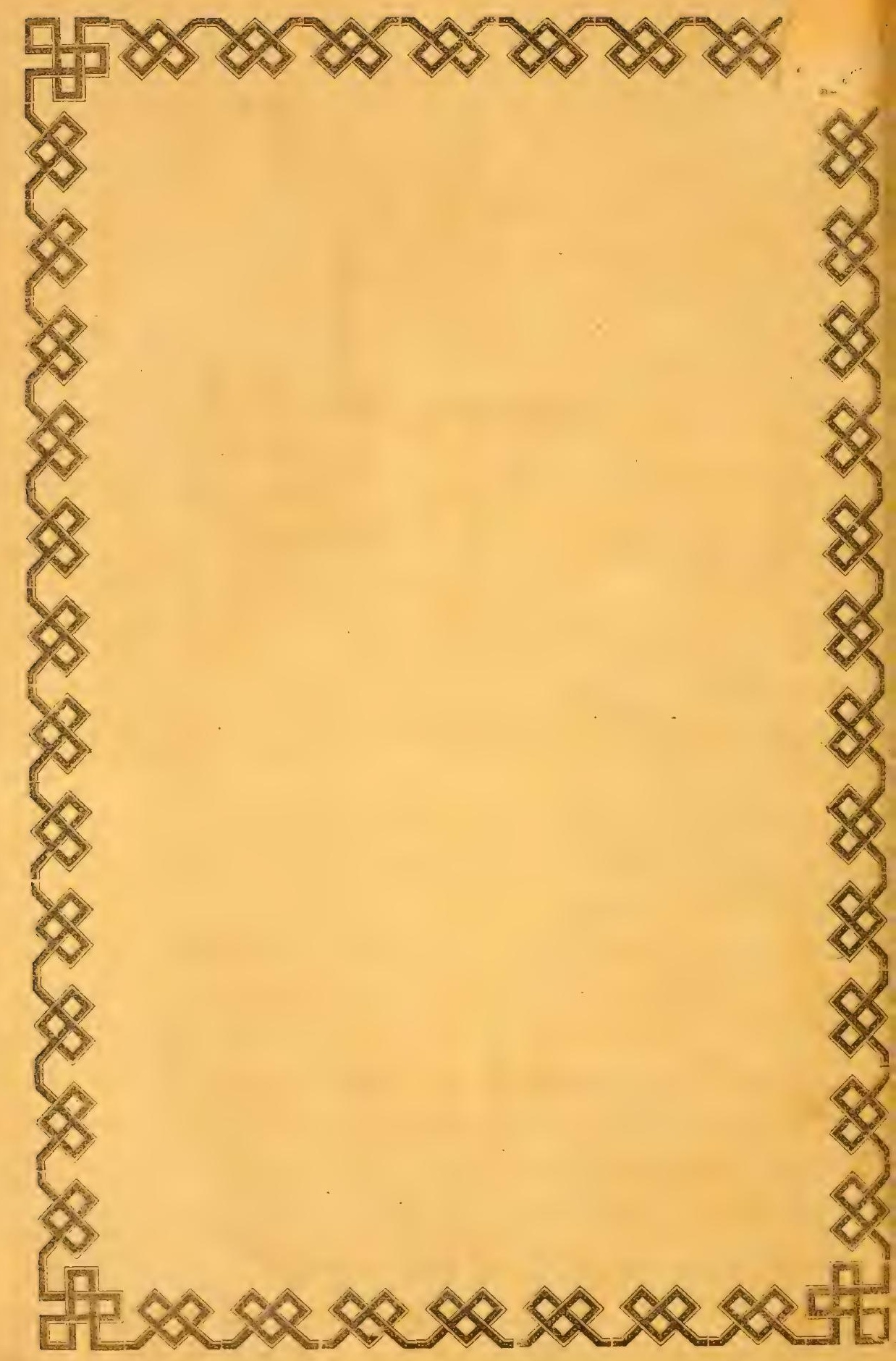






SMITHSONIAN INSTITUTION LIBRARIES

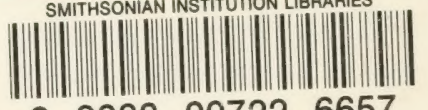

$\begin{array}{llll}3 & 9088 & 00722 \quad 6657\end{array}$ 